\title{
Statistical analysis of modulated codes for robot positioning - Application to BeAMS
}

\author{
Vincent Pierlot and Marc VAn Droogenbroeck
}

\begin{abstract}
Positioning is a fundamental issue for mobile robots. Therefore, a performance analysis is suitable to determine the behavior of a system, and to optimize its working. Unfortunately, some systems are only evaluated experimentally, which makes the performance analysis and design decisions very unclear.

In [4], we have proposed a new angle measurement system, named BeAMS, that is the key element of an algorithm for mobile robot positioning. BeAMS introduces a new mechanism to measure angles: it detects a beacon when it enters and leaves an angular window. A theoretical framework for a thorough performance analysis of BeAMS has been provided to establish the upper bound of the variance, and to validate this bound through experiments and simulations. It has been shown that the estimator derived from the center of this angular window provides an unbiased estimate of the beacon angle.

This document complements our paper by going into further details related to the code statistics of modulated signals in general, with an emphasis on BeAMS. In particular, the probability density function of the measured angle has been previously established with the assumption that there is no correlation between the times a beacon enters the angular window or leaves it. This assumption is questionable and, in this document, we reconsider this assumption and establish the exact probability density function of the angle estimated by BeAMS (without this assumption).

The conclusion of this study is that the real variance of the estimator provided by BeAMS was slightly underestimated in our previous work. In addition to this specific result, we also provide a new and extensive theoretical approach that can be used to analyze the statistics of any angle measurement method with beacons whose signal has been modulated.

To summarize, this technical document has four purposes:

(1) to establish the exact probability density function of the angle estimator of BeAMS,

(2) to calculate a practical upper bound of the variance of this estimator, which is of practical interest for calibration and tracking (see Table 1, on page 13, for a summary),

(3) to present a new theoretical approach to evaluate the performance of systems that use modulated (coded) signals, and

(4) to show how the variance evolves exactly as a function of the angular window (while remaining below the upper bound).
\end{abstract}

Keywords: angle, angle measurement, positioning, mobile robot, infrared signal, code statistics, estimator, variance, probability density function 


\section{Contents}

1 Introduction $\quad 3$

1.1 Principle of the system $($ summary $) \ldots \ldots \ldots \ldots$

1.2 Beacon identifier and infrared codes . . . . . . . . . . . . . . . 4

1.3 Source of errors . . . . . . . . . . . . . . . . . . . 6

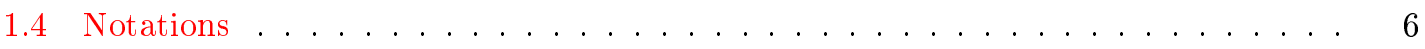

2 Probability density functions $\quad \mathbf{8}$

2.1 Probability density function of $\Phi_{r} \ldots \ldots \ldots \ldots$. . . . . . . . . . . 8

2.2 Probability density function of $\Phi_{f} \ldots \ldots \ldots$. . . . . . . . . . . . . 9

2.3 Characteristics of the estimators $\Phi_{r}$ and $\Phi_{f} \ldots \ldots \ldots \ldots$

3 Characterization of the estimator $\Phi_{b} \quad 9$

3.1 Mean of $\Phi_{b} \ldots \ldots \ldots \ldots \ldots \ldots$

3.2 Variance of $\Phi_{b} \ldots \ldots \ldots \ldots \ldots$

3.3 Computation of the upper bound of $\sigma_{\Phi_{b}}^{2} \ldots \ldots \ldots \ldots \ldots$

3.3.1 Modified random variables . . . . . . . . . . . . . 11

3.3.2 Towards a more realistic upper bound on $\sigma_{\Phi_{b}}^{2} \ldots \ldots \ldots$. . . . . . . 12

4 Evolution of the variance of $\Phi_{b}$ with respect to the angular window 14

4.1 Introduction to several situations . . . . . . . . . . . . . . . . 14

4.2 Study of the different cases . . . . . . . . . . . . . . . . . . 15

4.2 .1 The case A . . . . . . . . . . . . . . . . 17

4.2 .2 The case $0 \ldots \ldots \ldots \ldots \ldots$

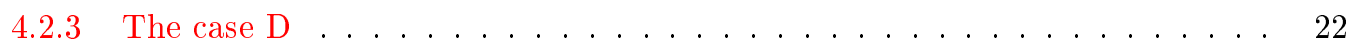

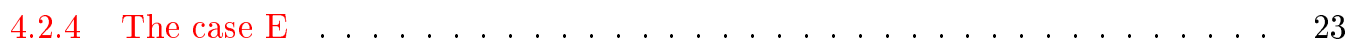

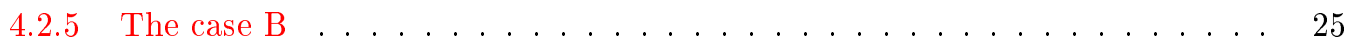

4.2 .6 The case $\mathrm{C} \ldots \ldots \ldots \ldots \ldots$

4.3 Summary of the variance value for all the cases . . . . . . . . . . . . . . 28

4.4 Simulations . . . . . . . . . . . . . . . . . . . . . 29

4.5 Note about the time stationarity hypothesis . . . . . . . . . . . . . . 32

5 Conclusions $\quad 36$

$\begin{array}{lr}\text { A Some details } & \mathbf{3 8}\end{array}$

A.1 Mean and variance of a random variable whose $P D F$ is expressed as a weighted sum (mixture) of PDFs . . . . . . . . . . . . . . . 38

A.2 Means and variances of $\Phi_{r}$ and $\Phi_{f} \ldots \ldots \ldots$. . . . . . . . . . . . . . . 39

A.2.1 Mean and variance of $\Phi_{r} \ldots \ldots \ldots \ldots$. . . . . . . . . . . . . . . . . . . . . . . . . . . . . . . . .

A.2.2 Mean and variance of $\Phi_{f} \ldots \ldots \ldots \ldots$. . . . . . . . . . . . . . . . . . . . . . . . . 40

A.3 Details for the case A . . . . . . . . . . . . . . . . . . . 40

A.4 Details for the case $0 \ldots \ldots \ldots$. . . . . . . . . . . . . . . 42

A.5 Details for the case D . . . . . . . . . . . . . . . . . 45

A.6 Details for the case $\mathrm{E} \ldots \ldots \ldots \ldots$. . . . . . . . . . . . . . . 48

A.7 Details for the case B . . . . . . . . . . . . . . . 50

A.8 Details for the case $\mathrm{C} \ldots \ldots \ldots \ldots \ldots \ldots$. . . . . . . . . . . . 52 


\section{Introduction}

In [2, 4], we have presented BeAMS, a new beacon based angle measurement system used for mobile robot positioning. This report elaborates on the errors committed on the measured angles, due to the codes. In other words, we focus on the artificial noise due to the code. We provide further and detailed results on the random variables representing these measured angles.

How to read this technical document? The reader not familiar with the BeAMS system may read Section 3 of [4] as an introduction, in order to have detailed explanations about the hardware and the functioning of the system. However, a brief summary of our system is presented in Section 1.1. The reader familiar with our system may jump directly to Section 3.1. The reader interested in the computation of the upper bound of the variance may read Section 3.3 only.

\subsection{Principle of the system (summary)}

BeAMS is composed of a sensor located on the robot, and several active beacons emitting infrared light in the horizontal plane and located at know locations. This configuration is drawn in Figure 1 .

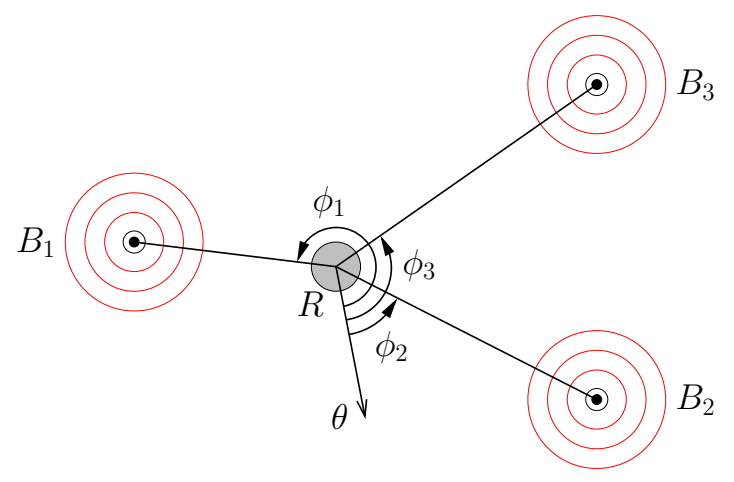

Figure 1: Schematic top view representation of the system. The system is composed of: (1) several active beacons $B_{i}$ emitting infrared light in the horizontal plane, and (2) a sensor located on the robot $R$. The aim of the sensor is to measure the azimuthal angles $\Phi_{i}$ of the beacons in the robot referential determined by $\theta$.

As illustrated in this figure, the aim of the sensor and processing unit is to determine the identifier of each beacon $i$, as well as their azimuthal angle $\Phi_{i}$, in the robot referential, whose orientation is given by $\theta$. Note that BeAMS can detect any number of beacons, even if, for illustrative purposes, we have only represented 3 beacons $^{1}$ in Figure 1.

The sensor is composed of an infrared receiver/demodulator and a motor. To achieve the angle measurements, the infrared receiver is combined through optical components to the motor, which turns at a constant speed. As a result, the receiver can virtually turn at the same speed as the motor. One of the key elements of our system is that the receiver sweeps the horizontal plane at constant speed, so that there is a perfect relationship between the angle and time.

The core of a beacon is composed of IR LEDs, which emit signals in a plane parallel to the moving area. In order to identify beacons and to increase robustness against noise, each beacon sends out a unique On-Off Keying (OOK) amplitude modulated signal over a $455 \mathrm{kHz}$ carrier frequency. But, because of our choice to avoid a synchronization channel between the beacons and the robot, each

\footnotetext{
${ }^{1}$ The number 3 is the minimal number of beacons required for triangulation. Therefore, in practice, most triangulation systems use 3 or more beacons (see [5] for more details on triangulation).
} 


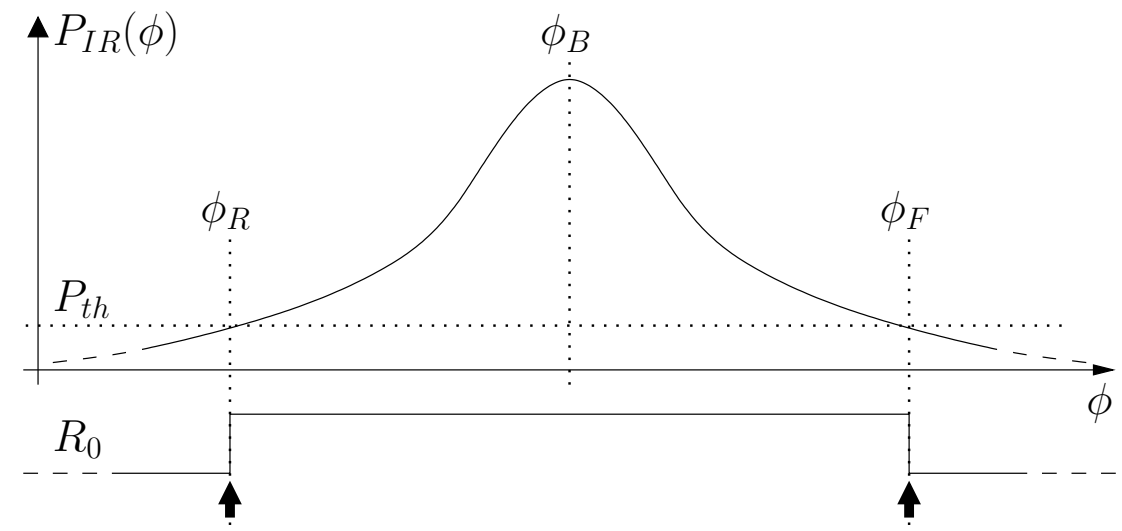

Figure 2: The upper curve $P_{I R}(\phi)$ is the expected infrared power collected at the receiver while the turret is turning. $R_{0}$ represents the receiver output for a non modulated infrared carrier wave (pure $455 \mathrm{kHz}$ sine wave). The black arrows represent the measured values respectively for $\phi_{R}$ to the left (first Rising edge) and for $\phi_{F}$ to the right (last Falling edge).

beacon has to continuously emit its own IR signal. As a result, each beacon signal is a periodic signal whose period corresponds to a particular code defining the beacon's ID.

The principle of the angle measurement is as follow. By design, the receiver combined with the optical components has a narrow field of view and, consequently, the amount of infrared power collected at the receiver, denoted by $P_{I R}(\phi)$, depends on the angle. The resulting expected curve $P_{I R}(\phi)$ is shown in Figure 2. First, let us assume that the beacons send a non modulated IR signal, that is a pure $455 \mathrm{kHz}$ sine wave and explain the measurement principle for one beacon (the principle is the same for any number of beacons). While the turret is turning, the receiver begins to "see" the IR signal from that beacon when the power threshold $P_{t h}$ is crossed upwards $(0 \rightarrow 1$ transition $)$. The receiver continues to receive that signal until $P_{t h}$ is crossed downwards ( $1 \rightarrow 0$ transition). The receiver output is depicted as $R_{0}$ in Figure 2 . At these transitions, the capture module latches values for $\phi_{R}$ and $\phi_{F}$. The angular position of the beacon is then computed as

$$
\phi_{B}=\frac{\phi_{R}+\phi_{F}}{2}
$$

\subsection{Beacon identifier and infrared codes}

The convenient assumption of continuous IR signals used in the previous section is not realistic because (1) we would not be able to distinguish between the different beacons, and (2) it is essential to establish the beacon's ID (especially in a very noisy environment like the EUROBoT contest where other IR sources may exist).

In BeAMS, each beacon periodically emits its own code over the $455 \mathrm{kHz}$ carrier wave; this emission is continuous so as to avoid having any form of synchronization between the beacons and the receiver. As a result, each beacon signal is a periodic signal whose period corresponds to a particular code defining the beacon's ID. The design of these codes is subject to several constraints related to (1) the receiver characteristics, (2) the loop emission, (3) the desired precision, (4) the system's immunity against noise, and (5) the number of beacons. We elaborate on these constraints below:

1. Receiver. The TSOP7000 requires that the burst length (presence of carrier wave) be chosen between 22 and $500 \mu \mathrm{s}$, the maximum sensitivity being reached with 14 carrier wave periods $(14 / 455000=30.8 \mu \mathrm{s})$. The gap time between two consecutive bursts (lack of carrier wave) should be at least $26 \mu \mathrm{s}$. 


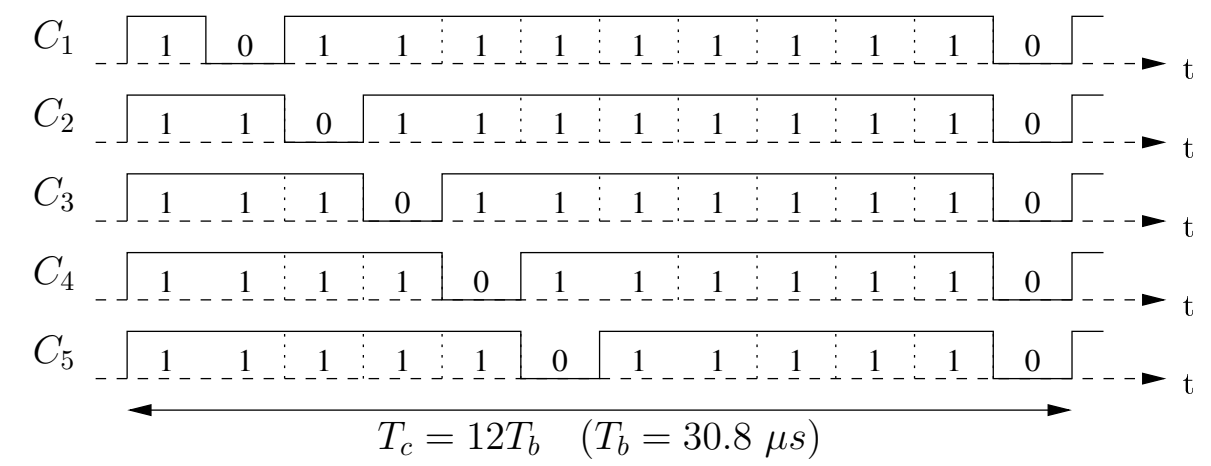

Figure 3: Temporal representation of the $C_{1}, C_{2}, C_{3}, C_{4}$ and, $C_{5}$ codes. These codes are repeated continuously and multiply the $455 \mathrm{kHz}$ carrier wave to compose the complete IR signal.

2. Loop emission. Because of our willingness to avoid a synchronization between beacons and the receiver, we must ensure that the periodic emission of a code does not introduce ambiguities. For example [0101] is equivalent to [1010] when sent in a loop. Thus any rotation of any code on itself must be different from another code.

3. Precision. The lack of synchronization between beacons and the receiver introduces a certain amount of imprecision. Indeed, the first received IR pulse may be preceded by a gap time corresponding to a zero symbol. This affects the estimation of $\phi_{R}$. The same phenomenon occurs for $\phi_{F}$. A fairly obvious and intuitive design rule would say that we have to reduce the duration of zeros, as well as their frequency of appearance. Therefore, we forbid two or more consecutive zeros, and the duration of one zero (the gap time) must be reduced as much as possible.

4. Immunity. The codes should contain enough redundancy to be robust against noise or irrelevant IR signals.

5. Number of beacons. The codes should be long enough to handle a few beacons, but as short as possible to be seen many times in the angular window associated to a beacon, thus improving the robustness of the decoding.

All these constraints lead us to propose this family of codes

$$
C_{i}=\left[1^{i} 0^{1} 1^{2 N_{c}-i} 0^{1}\right] \quad i=1, \ldots, N_{c}
$$

where $N_{c}$ denotes the number of codes in the system. The duration of a bit is set to $T_{b}=$ $30.8 \mu \mathrm{s}$ since this value maximizes the receiver sensitivity, while respecting the minimum gap time. Although not mandatory, the duration of a one symbol has been chosen to be equal to the duration of a zero. This is to simplify the implementation of the beacons and to ease the decoding process. The gap time is the same for all the codes and corresponds to the duration of a unique zero symbol. The second half part of the codes can be seen as a checksum, since it makes the number of ones constant $\left(2 N_{c}\right)$. In our current implementation, we have $N_{c}=5$ codes because this is appropriate for our application. Figure 3 shows the temporal representation of the codes for $N_{c}=5$. Note that any code meeting the second requirement (differentiable under loop emission) would work to identify the beacons. However, they may not meet the third requirement if the zero symbols appear in random patterns. From our experience, the codes presented in expression (2) are the best ones that meet all our requirements, but we have no formal proof of it.

The angle measurement principle still operates exactly as explained previously, even if the IR carrier wave is modulated by the codes. Since there are gap times in the IR signal of a beacon, there are more than two edges in the received signal. The intermediate edges are used to determine the beacon ID, by analyzing the durations of burst lengths and gap times. But the first and last edges of the received signal always correspond to our measurements of $\phi_{R}$ and $\phi_{F}$. These two edges are isolated from all other edges due to a timeout strategy, which relies on the fact that the separation time (or angle) between two different beacons is much greater than the separation time 
between consecutive edges in a code. Actually, the separation time is set to four bit durations, which corresponds to a separation angle equal to $0.44 \mathrm{deg}$.

\subsection{Source of errors}

As the receiver captures an OOK amplitude modulated signal, it can only detect the presence of the carrier wave (denoted by a 1 or ON period) or the absence of the carrier wave (denoted by a 0 or OFF period). If the carrier were sent continuously (that is, if the signal sent by a beacon was not coded), there would be no OFF periods in the signal captured by the receiver. But the coding of the beacon signals introduces 0's into the emitted sequences.

Let us now examine the influence of the OFF periods on the first rising and last falling edges measured by the system. Since there are errors in the system, there are no means to access the true values of $\phi_{R}$ and $\phi_{F}$. Therefore, we consider random variables instead, denoted by $\Phi_{r}$ and $\Phi_{f}$. According to (1), we propose the following estimator $\Phi_{b}$ for the beacon angular position $\phi_{B}$

$$
\Phi_{b}=\frac{\Phi_{r}+\Phi_{f}}{2} .
$$

As illustrated in Figure 4, if a beacon emits a 1 when it enters into the receiver's field of view, there is no error on $\Phi_{r}$. However, if a beacon emits a 0 when it enters the receiver's field of view, there is an error on $\Phi_{r}$ because no signal produces a $0 \rightarrow 1$ transition at the receiver output. In fact the transition occurs later $\left(\Phi_{r} \geq \phi_{R}\right)$, at the next 1 . The same consideration applies to $\Phi_{f}$, except that the $1 \rightarrow 0$ transition could occur sooner $\left(\Phi_{f} \leq \phi_{F}\right)$. All these specific situations are illustrated in Figure 4. We first represent the output of the receiver for a non modulated carrier wave, $R_{0}$. In that case, there are no errors in the transition times because the beacon sends out a continuous 1 symbol. The four other cases represent the output of the receiver for four different situations using an arbitrary code (we use here a simpler code than ours for the purpose of illustration, but this does not change the conclusions). The first case, corresponding to the received signal $R_{1}$, does not induce any error because $P_{t h}$ is reached upwards and downwards when the beacon emits a 1 symbol. The second case $\left(R_{2}\right)$ generates an error on $\Phi_{r}$ only. The third case $\left(R_{3}\right)$ generates an error on $\Phi_{f}$ only, and the fourth case $\left(R_{4}\right)$ generates an error on both $\Phi_{r}$ and $\Phi_{f}$.

Assume now that the OFF periods of a sequence all have the same duration, denoted by $T_{0}$ (this is our choice by design). Because the motor rotates at a constant speed, an OFF period is then equivalent to an OFF angle called $\phi_{0}$. The worst case for estimating $\Phi_{r}$ occurs when an OFF period starts at an angle $\phi=\phi_{R}$, delaying the next transition to an angle $\phi_{R}+\phi_{0}$. The same reasoning applies to $\Phi_{f}$ when an OFF period begins at an angle $\phi=\phi_{F}-\phi_{0}$. In both cases, the maximum absolute error on $\Phi_{r}$ or $\Phi_{f}$ is equal to $\phi_{0}$. These are the worst cases but there are many combinations of these two errors. In the following sections, we establish the probability density functions $(P D F s)$ of the random variables $\Phi_{r}$ and $\Phi_{f}$, and deduce characteristics of the estimator $\Phi_{b}$.

\subsection{Notations}

In the following, we use these notations:

- $N_{0}, N_{1}$ are the number of 0's or 1's in a code, respectively. The number of bits in a code is $N_{b}=N_{0}+N_{1}$.

- $p_{0}, p_{1}$ are the probabilities of obtaining a 0 or a 1 respectively at the IR power threshold (rising or falling edge), that is their frequencies. By definition, we have $p_{1}=N_{1} / N_{b}, p_{0}=$ $N_{0} / N_{b}$, and $p_{0}+p_{1}=1$.

- $T_{0}$ is the OFF period (duration of a 0 ) in a code. The only assumption is that the OFF periods of a code must all have the same duration. 


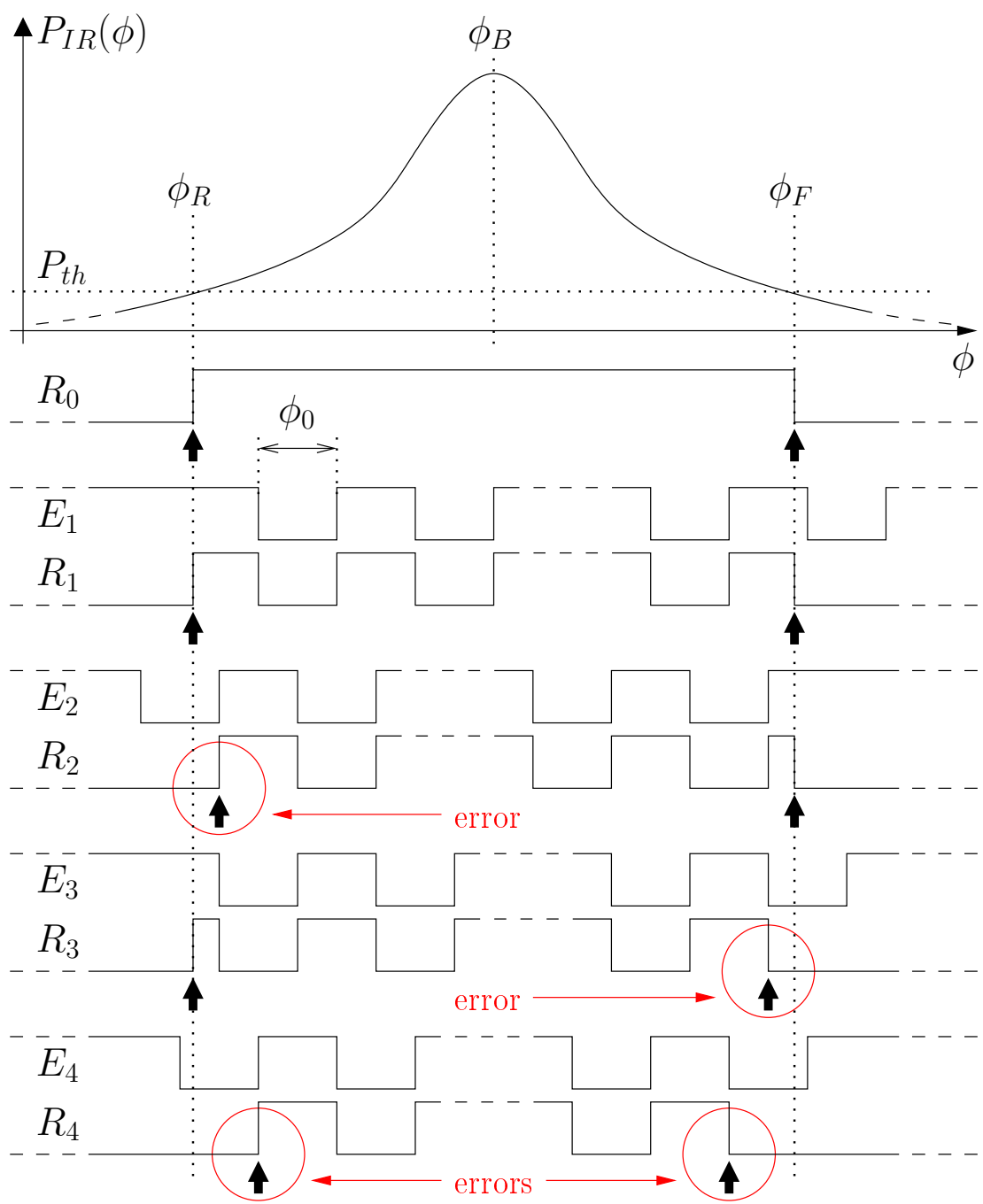

Figure 4: The upper curve $P_{I R}(\phi)$ is the infrared power collected at the receiver while the turret is turning. $E_{i}$ are examples of emitted signals from the beacons. $R_{i}$ are the corresponding received signals at the receiver output. $R_{0}$ is the special case corresponding to the non modulated infrared carrier wave (no OFF periods). The black arrows represent the measured values respectively for $\Phi_{r}$ to the left (first Rising edge) and for $\Phi_{f}$ to the right (last Falling edge). The encircled arrows emphasize errors made on $\Phi_{r}$ or $\Phi_{f}$. 
- $\phi_{0}$ is the OFF angle, corresponding to the OFF period $T_{0}$

$$
\phi_{0}=\omega T_{0} .
$$

To give an example of real values, in the original setup of our system for mobile robot positioning, we have $N_{1}=10, N_{0}=2, p_{0}=1 / 6, T_{0}=30.8 \mu \mathrm{s}$, and $\phi_{0}=0.111 \mathrm{deg}$, for each code.

- The Uniform $P D F$ is defined as

$$
U_{(a, b)}(x)= \begin{cases}\frac{1}{b-a} & \text { if } a \leq x \leq b \\ 0 & \text { otherwise }\end{cases}
$$

whose mean is $\frac{a+b}{2}$ and variance is $\frac{(b-a)^{2}}{12}$.

- The symmetric Triangular PDF is defined as

$$
T_{(a, b)}(x)= \begin{cases}\frac{2}{b-a}-\frac{2|2 x-a-b|}{(b-a)^{2}} & \text { if } a \leq x \leq b, \\ 0 & \text { otherwise }\end{cases}
$$

where $|x|$ denotes the absolute value of $x$ and whose mean is $\frac{a+b}{2}$ and variance is $\frac{(b-a)^{2}}{24}$. Note that, with these notations, and if $b-a=d-c$, we have [1, page 137]

$$
U_{(a, b)}(x) \otimes U_{(c, d)}(x)=T_{(a+c, b+d)}(x),
$$

where $\otimes$ denotes the convolution product.

\section{Probability density functions}

BeAMS introduces a new mechanism for measuring angles. To the contrary of systems that look for a maximum to estimate the angle of a beacon, BeAMS detects a beacon when it enters and when it leaves the angular window. Therefore, we have two random variables, $\Phi_{r}$ and $\Phi_{f}$, corresponding to these events. The estimator of the angle position, $\Phi_{b}$, is the mean of these two variables.

In this section, we establish the probability density functions of $\Phi_{r}, \Phi_{f}$, and $\Phi_{b}$. Obviously, there are some symmetries for $\Phi_{r}$ and $\Phi_{f}$; we will use them to shorten some developments.

\subsection{Probability density function of $\Phi_{r}$}

Errors on $\Phi_{r}$ originate if a beacon emits a 0 symbol while entering the angular window. Assuming time stationarity and as there is no synchronization between the beacons and the receiver, $p_{1}$ is the probability of determining the correct angle $\phi_{R}$ as the measured value for $\Phi_{r}$, when the beacon enters the angular window. When the beacon emits a 0 , the value measured for $\Phi_{r}$ is not correct; we then assume that its value is uniformly distributed between $\phi_{R}$ and $\phi_{R}+\phi_{0}$. Therefore, if we define $\delta(x)$ as the DiRAc delta function, then the $P D F$ of $\Phi_{r}$ is given by the following mixture of PDFs

$$
f_{\Phi_{r}}(\phi)=p_{1} \delta\left(\phi-\phi_{R}\right)+p_{0} U_{\left(\phi_{R}, \phi_{R}+\phi_{0}\right)}(\phi)
$$

for $\phi \in[-\pi, \pi)$. The mean and variance of $\Phi_{r}$ are, respectively,

$$
\begin{aligned}
\mu_{\Phi_{r}} & =\phi_{R}+p_{0} \frac{\phi_{0}}{2}, \\
\sigma_{\Phi_{r}}^{2} & =p_{0} \frac{\phi_{0}^{2}}{3}-p_{0}^{2} \frac{\phi_{0}^{2}}{4} .
\end{aligned}
$$

The details of the calculus can be found in Section A.2.1. 


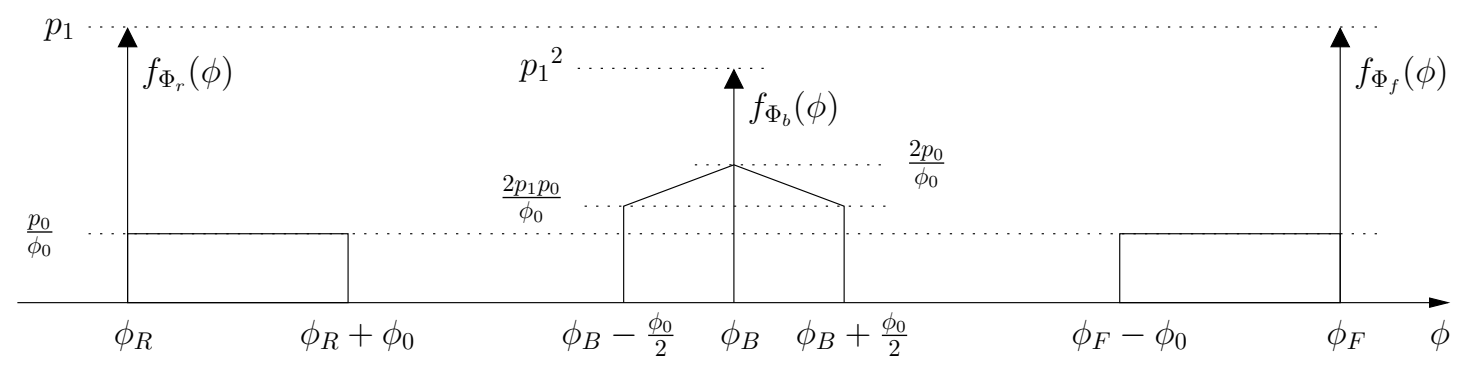

Figure 5: Probability density functions of $\Phi_{r}$ (left), $\Phi_{f}$ (right) and $\Phi_{b}$ (center) in the case of independent $\Phi_{r}$ and $\Phi_{f}$.

\subsection{Probability density function of $\Phi_{f}$}

Because the configuration is symmetric when the beacon exits the angular window, a similar result yields for $\Phi_{f}$

$$
f_{\Phi_{f}}(\phi)=p_{1} \delta\left(\phi-\phi_{F}\right)+p_{0} U_{\left(\phi_{F}-\phi_{0}, \phi_{F}\right)}(\phi),
$$

for $\phi \in[-\pi, \pi)$. The mean and variance of $\Phi_{f}$ are

$$
\begin{aligned}
\mu_{\Phi_{f}} & =\phi_{F}-p_{0} \frac{\phi_{0}}{2}, \\
\sigma_{\Phi_{f}}^{2} & =p_{0} \frac{\phi_{0}^{2}}{3}-p_{0}^{2} \frac{\phi_{0}^{2}}{4} .
\end{aligned}
$$

The details of the calculus can be found in Section A.2.2.

\subsection{Characteristics of the estimators $\Phi_{r}$ and $\Phi_{f}$}

The PDFs of $\Phi_{r}$ and $\Phi_{f}$ are drawn in Figure 5. The expectations of $\Phi_{r}$ and $\Phi_{f}$ have a bias given by $\pm p_{0} \frac{\phi_{0}}{2}$ respectively (see equations (9) and (12)). The bias is proportional to the OFF angle $\phi_{0}$ and the proportion of 0 's in a code $p_{0}$. The variances of $\Phi_{r}$ and $\Phi_{f}$ are equal, and they are equal to zero if and only if there is no OFF period in the codes ${ }^{2}$.

\section{Characterization of the estimator $\Phi_{b}$}

\subsection{Mean of $\Phi_{b}$}

The aim of the system being to estimate the beacon angular position $\phi_{B}$, we are now interested in finding the mean and variance of $\Phi_{b}$. Generally the mean and variance of a random variable are calculated with the help of the $P D F$. In the case of $\Phi_{b}$, it is not necessary to derive them from the $P D F$ since the estimator is a function of $\Phi_{r}$ and $\Phi_{f}$ (equation (3)), whose PDFs are known. Let us first consider the mean of $\Phi_{b}$. For any random variables $X$ and $Y$, we have $E\{X+Y\}=E\{X\}+E\{Y\}$ (see for example [1, page 152]). Therefore, the mean of $\Phi_{b}$ is given

\footnotetext{
${ }^{2}$ OFF periods correspond to the absence of any signal. The presence of OFF periods is nevertheless necessary because we need to code the signal to identify beacons. If the identifier is not contained inside the signal (inband signaling), then we need an additional signal to obtain a beacon's identifier (out-band signaling). Out-band signaling systems are more complex and have their own problems, not easy to solve for mobile robot positioning. BeAMS uses in-band signaling.
} 
by

$$
\begin{aligned}
\mu_{\Phi_{b}} & =\frac{E\left\{\Phi_{r}\right\}+E\left\{\Phi_{f}\right\}}{2}, \\
& =\frac{\left(\phi_{R}+p_{0} \frac{\phi_{0}}{2}\right)+\left(\phi_{F}-p_{0} \frac{\phi_{0}}{2}\right)}{2}, \\
& =\frac{\phi_{R}+\phi_{F}}{2}=\phi_{B} .
\end{aligned}
$$

As can be seen, the mean of $\Phi_{b}$ is unbiased, despite that both the entering angle $\Phi_{r}$ and leaving angle $\Phi_{f}$ estimators are biased. This justifies the construction of a symmetric receiver and the use of that estimator.

\subsection{Variance of $\Phi_{b}$}

Let us now derive the variance of $\Phi_{b}$. The variance of the sum of two random variables can be expanded as [1]

$$
\sigma_{\Phi_{b}}^{2}=\operatorname{var}\left\{\frac{\Phi_{r}+\Phi_{f}}{2}\right\}=\frac{\operatorname{var}\left\{\Phi_{r}+\Phi_{f}\right\}}{4}=\frac{\sigma_{\Phi_{r}}^{2}+\sigma_{\Phi_{f}}^{2}+2 C\left\{\Phi_{r}, \Phi_{f}\right\}}{4} .
$$

where $C\left\{\Phi_{r}, \Phi_{f}\right\}$ denotes the covariance of $\Phi_{r}$ and $\Phi_{f}$. If $\Phi_{r}$ and $\Phi_{f}$ are uncorrelated, we have that [1, page 155]

$$
\sigma_{\Phi_{b}}^{2}=\frac{\sigma_{\Phi_{r}}^{2}+\sigma_{\Phi_{f}}^{2}}{4}=\frac{\sigma_{\Phi_{r}}^{2}}{2}=\frac{\sigma_{\Phi_{f}}^{2}}{2}
$$

since $\sigma_{\Phi_{r}}^{2}=\sigma_{\Phi_{f}}^{2}$. This could also have been derived from the PDF of $\Phi_{b}$, that is given by, in the case of independent $\Phi_{r}$ and $\Phi_{f}$,

$$
f_{\Phi_{b}}(\phi)=p_{1}^{2} \delta\left(\phi-\phi_{B}\right)+2 p_{1} p_{0} U_{\left(\phi_{B}-\frac{\phi_{0}}{2}, \phi_{B}+\frac{\phi_{0}}{2}\right)}(\phi)+p_{0}^{2} T_{\left(\phi_{B}-\frac{\phi_{0}}{2}, \phi_{B}+\frac{\phi_{0}}{2}\right)}(\phi) .
$$

This result is obtained by convolving the PDFs of $\Phi_{r}$ and $\Phi_{f}$ [1, page 136], using equation (7) and rescaling the result by using these properties [1]: 1) if $Y=\alpha X$, then $f_{Y}(y)=\frac{1}{|\alpha|} f_{X}\left(\frac{y}{\alpha}\right)$, and 2) $\delta(\alpha x)=\frac{1}{|\alpha|} \delta(x)$. This probability density function is also depicted in Figure 5 (center). However, the non correlation or independence of $\Phi_{r}$ and $\Phi_{f}$ are questionable in our case; this is discussed hereafter.

As explained earlier, four situations are possible in one angular window: (1) no error is encountered, (2) an error occurs for $\Phi_{r}$ only, (3) an error occurs for $\Phi_{f}$ only, or (4) an error occurs for both angles. But the codes are deterministic and not random, and the durations between OFF periods are fixed and known. So, depending on the rotating speed and the code, it is not sure that an error is possible on $\Phi_{r}$ and $\Phi_{f}$ simultaneously. These remarks show that the $\Phi_{r}$ and $\Phi_{f}$ variables are not independent, and that the nature of the relationship depends on the angular window and the coding scheme. To establish this relationship, we should analyze, in full details, the four previous cases in function of the angular window and the different codes. However, the mean of $\Phi_{b}$ is always given by equation (14), and despites the relationship between $\Phi_{r}$ and $\Phi_{f}, \Phi_{b}$ remains unbiased. To the contrary, the variance of $\Phi_{b}$ is no longer given by equation (16) when $\Phi_{r}$ and $\Phi_{f}$ are correlated.

Fortunately, it is possible to derive an upper bound for $\sigma_{\Phi_{b}}^{2}$ for a practical use, as we did in [3]. This result is reminded hereafter. Indeed, the square of the covariance is upper bounded [1, page $153]$

$$
C^{2}\left\{\Phi_{r}, \Phi_{f}\right\} \leq \sigma_{\Phi_{r}}^{2} \sigma_{\Phi_{f}}^{2} .
$$

Given that $\sigma_{\Phi_{r}}^{2}=\sigma_{\Phi_{f}}^{2}$, we combine equations (15) and (18) to establish the following limits for $\sigma_{\Phi_{b}}^{2}$ 


$$
0 \leq \sigma_{\Phi_{b}}^{2} \leq \sigma_{\Phi_{r}}^{2} .
$$

The upper bound of $\sigma_{\Phi_{b}}^{2}$ is given by

$$
\sigma_{\Phi_{b}}^{2} \leq \sigma_{\Phi_{r}}^{2}=\sigma_{\Phi_{f}}^{2}=p_{0} \frac{\phi_{0}^{2}}{3}-p_{0}^{2} \frac{\phi_{0}^{2}}{4} .
$$

The upper bound for $C\left\{\Phi_{r}, \Phi_{f}\right\}$ is a universal bound because it does not make any guess about a possible relationship between the random variables. This result is confirmed by the simulations but it seems to over estimate the real upper bound of $\sigma_{\Phi_{b}}^{2}$. Indeed, this first upper bound does not take into account the particular nature of the committed errors. The purpose of the following section is to provide a more accurate result.

\subsection{Computation of the upper bound of $\sigma_{\Phi_{b}}^{2}$}

With respect to the relationship between $\Phi_{r}$ and $\Phi_{f}$, three important points should be noted:

1. the PDFs of $\Phi_{r}$ and $\Phi_{f}$ remain correct, as well as their means and variances.

2. the estimator $\Phi_{b}$ remains unbiased, since the expectation does not depend on the relationship between variables.

3. the upper bound computed in [3] also remains correct, even if it tends to over estimate the variance of $\Phi_{b}$.

In order to understand the link between $\Phi_{r}$ and $\Phi_{f}$, and to find out a more accurate result on the upper bound of $\sigma_{\Phi_{b}}^{2}$, we have to analyze the four previous cases more cautiously in function of the angular window and the different codes. From a mathematical point of view, we need to compute the covariance $C\left\{\Phi_{r}, \Phi_{f}\right\}$ for all possible cases, and put the result back into equation (15). Note that the covariance can be expanded as [1, page 152]

$$
C\left\{\Phi_{r}, \Phi_{f}\right\}=E\left\{\Phi_{r}, \Phi_{f}\right\}-E\left\{\Phi_{r}\right\} E\left\{\Phi_{f}\right\} .
$$

Since $E\left\{\Phi_{r}\right\}$ and $E\left\{\Phi_{f}\right\}$ are known (see equations (9) and (12)), we need to compute the joint expectation $E\left\{\Phi_{r}, \Phi_{f}\right\}$. And to compute the joint expectation, we need to express the joint PDF $f_{\Phi_{r} \Phi_{f}}\left(\phi_{r}, \phi_{f}\right)$.

\subsubsection{Modified random variables}

In order to simplify the calculus, we define the modified random variables $\mathcal{E}_{r}$ and $\mathcal{E}_{f}$ as follows

$$
\begin{aligned}
\mathcal{E}_{r} & =\Phi_{r}-\phi_{R}, \\
\mathcal{E}_{f} & =\Phi_{f}-\phi_{F} .
\end{aligned}
$$

$\mathcal{E}_{r}$ and $\mathcal{E}_{f}$ are shifted versions of $\Phi_{r}$ and $\Phi_{f}$, by an amount equal to $\phi_{R}$, or $\phi_{F}$ respectively, and, as a result, they represent the errors committed on these measurements. The PDFs of these new random variables are

$$
\begin{gathered}
f_{\mathcal{E}_{r}}\left(\epsilon_{r}\right)=p_{1} \delta\left(\epsilon_{r}\right)+p_{0} U_{\left(0, \phi_{0}\right)}\left(\epsilon_{r}\right), \\
f_{\mathcal{E}_{f}}\left(\epsilon_{f}\right)=p_{1} \delta\left(\epsilon_{f}\right)+p_{0} U_{\left(-\phi_{0}, 0\right)}\left(\epsilon_{f}\right),
\end{gathered}
$$

and their expectations are

$$
\begin{aligned}
\mu_{\mathcal{E}_{r}} & =p_{0} \frac{\phi_{0}}{2}, \\
\mu_{\mathcal{E}_{f}} & =-p_{0} \frac{\phi_{0}}{2} .
\end{aligned}
$$


The variances are unaltered since

$$
\sigma_{\mathcal{E}_{r}}^{2}=\operatorname{var}\left\{\mathcal{E}_{r}\right\}=\operatorname{var}\left\{\Phi_{r}-\phi_{R}\right\}=\operatorname{var}\left\{\Phi_{r}\right\}=p_{0} \frac{\phi_{0}^{2}}{3}-p_{0}^{2} \frac{\phi_{0}^{2}}{4}=\sigma_{\mathcal{E}_{f}}^{2} .
$$

Finally, we have

$$
C\left\{\mathcal{E}_{r}, \mathcal{E}_{f}\right\}=C\left\{\Phi_{r}-\phi_{R}, \Phi_{f}-\phi_{F}\right\}=C\left\{\Phi_{r}, \Phi_{f}\right\}
$$

The variance of $\Phi_{b}$, expressed in terms of these new random variables $\mathcal{E}_{r}$ and $\mathcal{E}_{f}$, is then given by

$$
\begin{aligned}
\sigma_{\Phi_{b}}^{2} & =\frac{\sigma_{\Phi_{r}}^{2}+\sigma_{\Phi_{f}}^{2}+2 C\left\{\Phi_{r}, \Phi_{f}\right\}}{4} \\
& =\frac{\sigma_{\mathcal{E}_{r}}^{2}+\sigma_{\mathcal{E}_{f}}^{2}+2 C\left\{\mathcal{E}_{r}, \mathcal{E}_{f}\right\}}{4} \\
& =\frac{2 \sigma_{\mathcal{E}_{r}}^{2}+2 C\left\{\mathcal{E}_{r}, \mathcal{E}_{f}\right\}}{4} \\
& =\frac{\sigma_{\mathcal{E}_{r}}^{2}+C\left\{\mathcal{E}_{r}, \mathcal{E}_{f}\right\}}{2}
\end{aligned}
$$

By definition, the covariance of $\mathcal{E}_{r}$ and $\mathcal{E}_{f}$ is given by

$$
C\left\{\mathcal{E}_{r}, \mathcal{E}_{f}\right\}=E\left\{\mathcal{E}_{r}, \mathcal{E}_{f}\right\}-E\left\{\mathcal{E}_{r}\right\} E\left\{\mathcal{E}_{f}\right\}
$$

Therefore, we obtain

$$
\begin{aligned}
\sigma_{\Phi_{b}}^{2} & =\frac{\sigma_{\mathcal{E}_{r}}^{2}+E\left\{\mathcal{E}_{r}, \mathcal{E}_{f}\right\}-E\left\{\mathcal{E}_{r}\right\} E\left\{\mathcal{E}_{f}\right\}}{2} \\
& =\frac{\sigma_{\mathcal{E}_{r}}^{2}}{2}+\frac{E\left\{\mathcal{E}_{r}, \mathcal{E}_{f}\right\}}{2}-\frac{E\left\{\mathcal{E}_{r}\right\} E\left\{\mathcal{E}_{f}\right\}}{2} \\
& =p_{0} \frac{\phi_{0}^{2}}{6}-p_{0}^{2} \frac{\phi_{0}^{2}}{8}+\frac{E\left\{\mathcal{E}_{r}, \mathcal{E}_{f}\right\}}{2}+p_{0}^{2} \frac{\phi_{0}^{2}}{8} \\
& =p_{0} \frac{\phi_{0}^{2}}{6}+\frac{E\left\{\mathcal{E}_{r}, \mathcal{E}_{f}\right\}}{2} .
\end{aligned}
$$

The challenge is to compute the joint expectation $E\left\{\mathcal{E}_{r}, \mathcal{E}_{f}\right\}$

$$
E\left\{\mathcal{E}_{r}, \mathcal{E}_{f}\right\}=\iint_{-\infty}^{+\infty} \epsilon_{r} \epsilon_{f} f_{\mathcal{E}_{r} \mathcal{E}_{f}}\left(\epsilon_{r}, \epsilon_{f}\right) d \epsilon_{r} d \epsilon_{f}
$$

For this, we need to express $f_{\mathcal{E}_{r} \mathcal{E}_{f}}\left(\epsilon_{r}, \epsilon_{f}\right)$ for the different codes and angular windows.

\subsubsection{Towards a more realistic upper bound on $\sigma_{\Phi_{b}}^{2}$}

In order to compute a more realistic upper bound, we have to take into account the particular nature of the errors $\mathcal{E}_{r}$ and $\mathcal{E}_{f}$, and, more specifically, their bounds. A closer look onto the $\mathcal{E}_{r}$ and $\mathcal{E}_{f}$ definitions, or their PDFs shows that we have

$$
\begin{aligned}
0 & \leq \mathcal{E}_{r} \leq \phi_{0}, \\
-\phi_{0} & \leq \mathcal{E}_{f} \leq 0 .
\end{aligned}
$$

Graphically, it means that the joint $P D F$ of $\mathcal{E}_{r}$ and $\mathcal{E}_{f}$ is not null only inside a square of side $\phi_{0}$, as shown in Figure 6 (left). Moreover, their product is always negative or null

$$
\mathcal{E}_{r} \mathcal{E}_{f} \leq 0
$$



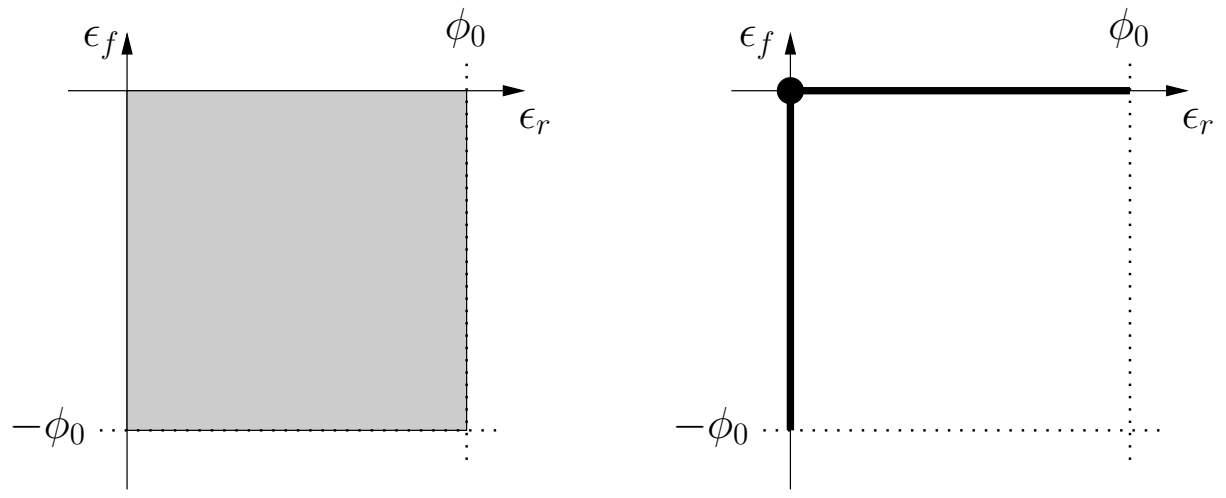

Figure 6: Left hand side: possible support of the joint $P D F$ of $\mathcal{E}_{r}$ and $\mathcal{E}_{f}$ in the general case. Right hand side: support of the joint $P D F$ of $\mathcal{E}_{r}$ and $\mathcal{E}_{f}$ when no error is possible on $\Phi_{r}$ and $\Phi_{f}$ simultaneously.

\begin{tabular}{|c|c|c|}
\hline & bound on $\sigma_{\Phi_{b}}$, as established in [3] & new upper bound on $\sigma_{\Phi_{b}}$ \\
\hline \hline expression & $\sigma_{\Phi_{b}} \leq \phi_{0} \sqrt{\frac{p_{0}}{3}-\frac{p_{0}^{2}}{4}}$ & $\sigma_{\Phi_{b}} \leq \phi_{0} \sqrt{\frac{p_{0}}{6}}$ \\
\hline numerical value & $0.0245 \mathrm{deg}$ & $0.0185 \mathrm{deg}$ \\
\hline
\end{tabular}

Table 1: Comparison of two bounds on $\sigma_{\Phi_{b}}$.

and so must be their joint expectation

$$
E\left\{\mathcal{E}_{r}, \mathcal{E}_{f}\right\} \leq 0
$$

Therefore

$$
\max E\left\{\mathcal{E}_{r}, \mathcal{E}_{f}\right\}=0,
$$

regardless of the relationship between $\mathcal{E}_{r}$ and $\mathcal{E}_{f}$.

We can therefore derive the following upper bound from expression (35),

$$
\sigma_{\Phi_{b}}^{2}=p_{0} \frac{\phi_{0}^{2}}{6}+\frac{E\left\{\mathcal{E}_{r}, \mathcal{E}_{f}\right\}}{2}
$$

Theorem 1 [Upper bound of $\sigma_{\Phi_{b}}^{2}$ ] For all our codes, the variance of $\Phi_{b}$ is bounded by $p_{0} \frac{\phi_{0}^{2}}{6}$

$$
\sigma_{\Phi_{b}}^{2} \leq p_{0} \frac{\phi_{0}^{2}}{6}
$$

Numerical values. In our case, $p_{0}=1 / 6$ and $\phi_{0}=0.111 \mathrm{deg}$, for each code. Previously, according to expression (20), the standard deviation was lower than $0.0245 \mathrm{deg}$. This new upper bound implies that $\sigma_{\Phi_{b}} \leq 0.0185 \mathrm{deg}$; this is a decrease of about $25 \%$. These results are summarized in Table 1.

As expected, the variance is related to the presence of OFF periods in the codes. More precisely, the variance is proportional to the probability of having a zero $p_{0}$, and to the square of the OFF angle $\phi_{0}$. It is equal to zero if and only if there is no OFF period in the codes.

Finally, it is interesting to interpret the condition $E\left\{\mathcal{E}_{r}, \mathcal{E}_{f}\right\}=0$, that is the maximum of the joint expectation. This constraint, combined to constraint (39) means that either $\mathcal{E}_{r}$ or $\mathcal{E}_{f}$ must be null. In other terms, it is impossible to make an error on both $\Phi_{r}$ and $\Phi_{f}$. Intuitively, it is logical that the variance is maximum in that case since an error committed on $\Phi_{r}$ is not balanced by an error on $\Phi_{f}$, and vice versa. Graphically, it means that the support of the joint $P D F$ reduces to 
the axes in that case. The possible support of the joint $P D F$ is represented in Figure 6 (right). In the next section, we show that it can be composed of uniform PDFs along the axes (the horizontal and vertical line segments) and a two dimensional DiRAC PDF (the black dot).

\section{Evolution of the variance of $\Phi_{b}$ with respect to the angular window}

In the previous section, we have computed the upper bound on $\Phi_{b}$. This upper bound is a general result for all codes with the sole assumption that the OFF periods of all codes must have the same duration.

But, if we can provide the upper bound for all codes, the previous analysis does not tell for which angular windows this upper bound is reached. To determine them, we need to consider both the particular code and angular window. More generally, we need to express $f_{\mathcal{E}_{r} \mathcal{E}_{f}}\left(\epsilon_{r}, \epsilon_{f}\right)$ for the different codes and angular windows. The final goal is to compute the variance of $\Phi_{b}$, for any code, and for any angular window. This is essential to estimate the precision of the estimator in order to feed a Kalman filter.

\subsection{Introduction to several situations}

In this section, we establish the evolution of the variance with respect to the angular window. It appears that we have to deal with 6 different situations, corresponding to:

Case 0. This case occurs when the angular window $\phi_{W}$ is lower than $\phi_{0}$, the OFF angle; that is $\phi_{W}=\lambda_{0} \phi_{0}$, with $\lambda_{0}<1$. It does not depend on any particular code. Errors are therefore bounded by the maximal value of the angular window $\left(\phi_{W}=\lambda_{0} \phi_{0}\right)$

$$
\begin{aligned}
0 & \leq \mathcal{E}_{r} \leq \lambda_{0} \phi_{0} \\
-\lambda_{0} \phi_{0} & \leq \mathcal{E}_{f} \leq 0
\end{aligned}
$$

The support of the joint $P D F$ is similar to the one represented in Figure 6 (right), except that the parts along the axes are smaller. The support of the joint $P D F$ is represented in Table 2, that regroups the PDFs of all the cases.

Case A. This case corresponds to the situation leading to a variance that is exactly equal to its upper bound, as explained in Section 3.3.2. It means that it is impossible to have $\mathcal{E}_{r} \neq 0$ and $\mathcal{E}_{f} \neq 0$ simultaneously, when the value of the angular window $\phi_{W}$ falls into given intervals, that depend on the particular code. The support of the joint $P D F$ is represented in Table 2.

Case B. This case is a combination of case A and case D (see below). It appears for some values of the angular window that depend on the code.

Case C. This case is a combination of case A and case E (see below). It appears for some values of the angular window that depend on the code.

Case D. In that case, it is possible to have $\mathcal{E}_{r} \neq 0$ and $\mathcal{E}_{f} \neq 0$ simultaneously, in addition to the three other situations of case 0 and case A (no error, $\mathcal{E}_{r} \neq 0$ only, $\mathcal{E}_{f} \neq 0$ only). But, when this situation occurs, the errors are totally dependent. In particular, their difference is constant along a part of the support of the joint $P D F$. It appears for values of $\phi_{W}$, that depend on the particular code. The support of the joint $P D F$ is represented in Table 2.

Case E. This case is similar to case D, meaning that along a part of the support of the joint $P D F$, the errors are totally dependent. But, unlike case $\mathrm{D}$, this situation occurs in another part of the joint $P D F$. It appears for values of $\phi_{W}$, that depend on the particular code. The support of the joint $P D F$ is represented in Table 2. 
These situations, named "Cases", have different supports for the joint PDF (as established later in this document); these supports are shown in Table 2. It is important to understand that, while the absolute error on $\mathcal{E}_{r}$ or $\mathcal{E}_{f}$ is $\phi_{0}$, the maximum error on $\Phi_{b}$ is not $\phi_{0}$ but $\phi_{0} / 2$ ! In other words, the errors on $\mathcal{E}_{r}$ and $\mathcal{E}_{f}$ do not sum up, but they compensate. This explains the particular shape of the domain of the different PDFs. Note that, while the support of the PDFs are continuous from a theoretical perspective, observed values are generally different for each revolution of the turret, and that successive observations move along the support of the PDFs. We elaborate on these aspects in subsection 4.5.

From a practical point of view, BeAMS operates with the best upper bound for $\Phi_{b}$ all over the plane (regardless of the robot position). But this practice is far from being optimal. Indeed, we show that the real variance evolves with the position of the robot in the plane, and therefore the upper bound overestimates the real variance unnecessarily. It would be better to have the exact variance for each position in the plane, and for each beacon (because the variances are related to the code and to the angular window).

It is essential to note that the variance $\sigma_{\Phi_{b}}^{2}$ is not a function of the distance to a beacon, but to the seen angular window. This might not be very intuitive, but this is coherent and more tractable for an angular measurement system that should not be sensitive to the distance. The major reason is that the relationship between the physical notion of distance and the measures is dependent on parameters, like power thresholds or propagation laws, that are out of control. The fact that $\sigma_{\Phi_{b}}^{2}$ is only dependent on the measured angular window and the beacon's code (which is known) is a huge advantage of BeAMS compared to other angle measuring systems.

In the following, we first establish the probability density function and variance for all the Cases. Then, we summarize them in Section 4.3. Then, we show the simulations results in Section 4.4.

\subsection{Study of the different cases}

Before starting the detailed study, one has to remember the general results obtained so far:

1. the upper bound is always valid, and corresponds to case A.

2. the mean of $\Phi_{b}$ remains unbiased, for all cases.

3. the marginal PDFs of the joint PDF are always given by equations (24) and (25), for all cases, except for the case 0.

In addition to these remarks, we have noted that:

1. the value of the variance of $\Phi_{b}$ is a function of the particular code, and the angular window $\phi_{W}$. The only constraint on $\phi_{W}$ is that it is positive or null by design $\left(\Phi_{f} \geq \Phi_{r}\right)$

$$
\phi_{W} \geq 0 \text {. }
$$

2. as the codes are periodic, the evolution of the variance w.r.t. $\phi_{W}$ for each code must be periodic. But, as a consequence of constraint (45), the function is not periodic in the strict mathematical sense. Indeed, we will show that this function is (partially/semi) periodic, for $\phi_{W} \geq \phi_{0}$ only

$$
\sigma_{\Phi_{b}}^{2}\left(\phi_{W}\right)=\sigma_{\Phi_{b}}^{2}\left(\phi_{W}+P\right) \quad \phi_{W} \geq \phi_{0},
$$

where the period $P$ is related to the length of a code: $P=12 \phi_{0}$.

3. in this study, we think in terms of angles instead of times since the gap time perceived by the system $\left(\phi_{0}=\omega T_{0}\right)$ is a combination of the real zero duration $\left(T_{0}\right)$ and the rotation speed of the turret $(\omega)$. Again, this is suitable for an angle measurement system like BeAMS.

4. the different cases are detailed hereafter in a different order than their logical name. Indeed, we detail them in order of complexity, and the way they are named will become clear later. 
Case 0

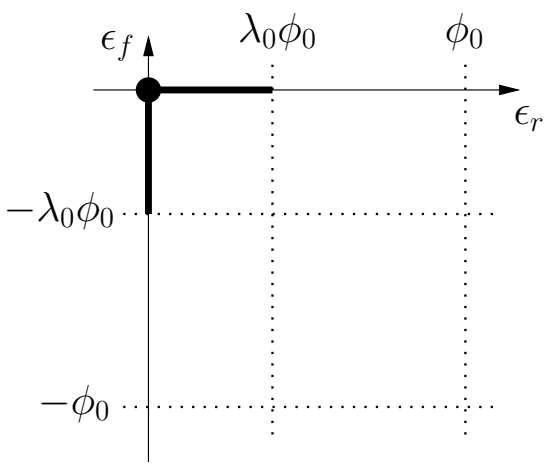

Case D

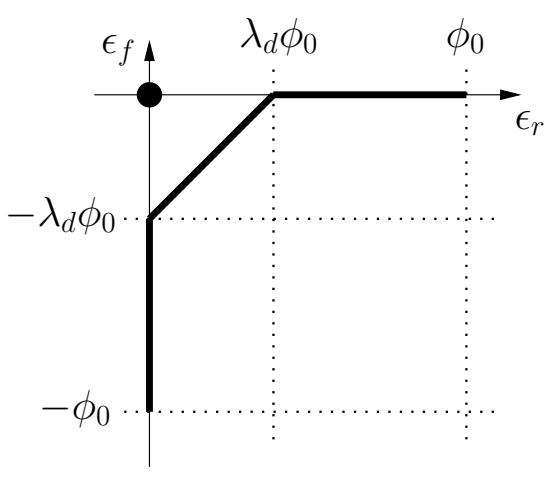

Case A

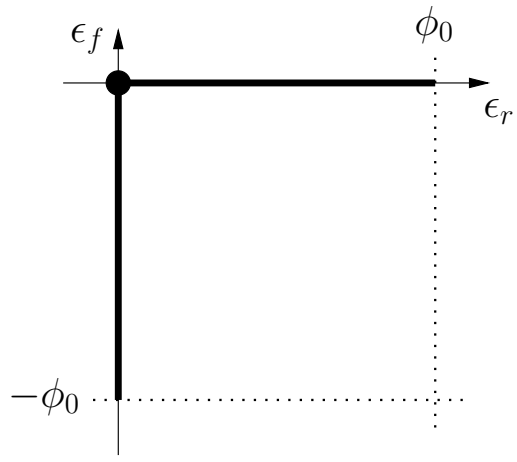

Case E

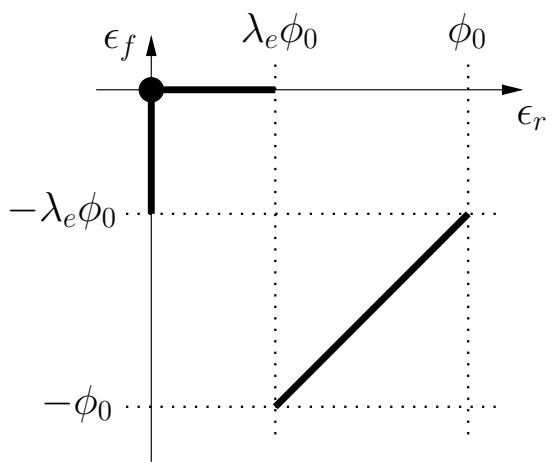

Case B
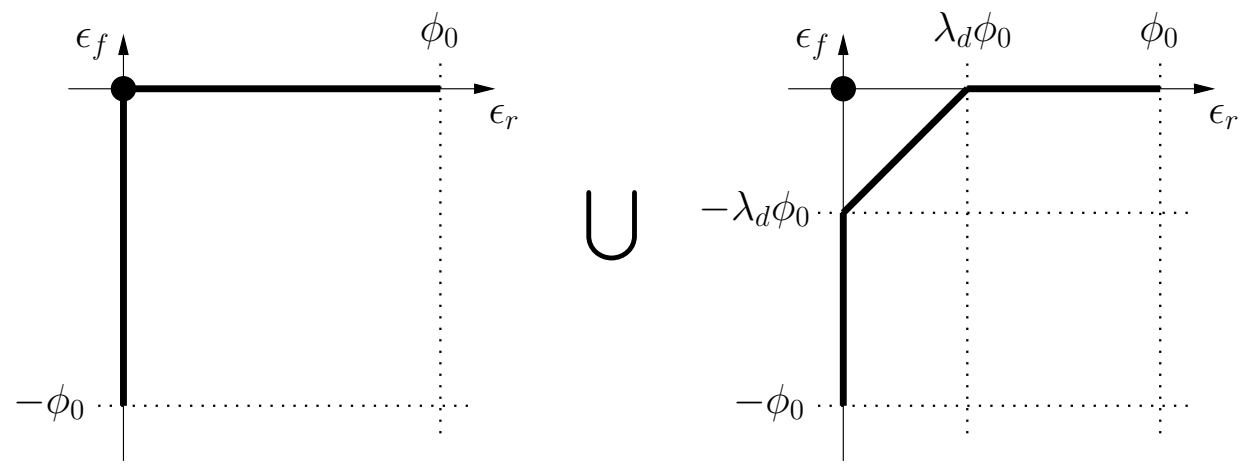

Case C
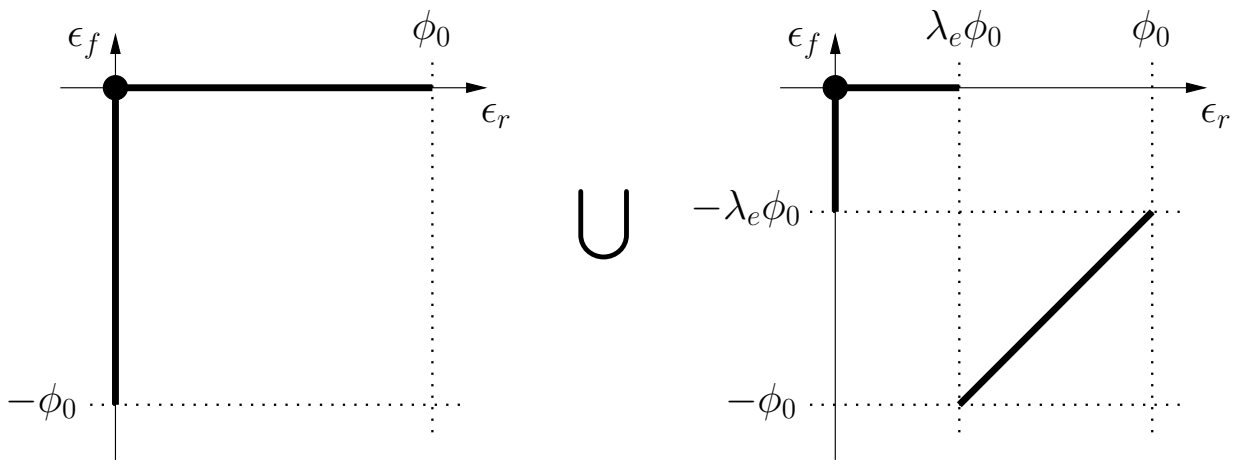

Table 2: Supports of the joint PDFs of $\mathcal{E}_{r}$ and $\mathcal{E}_{f}$ for the 6 different Cases. 


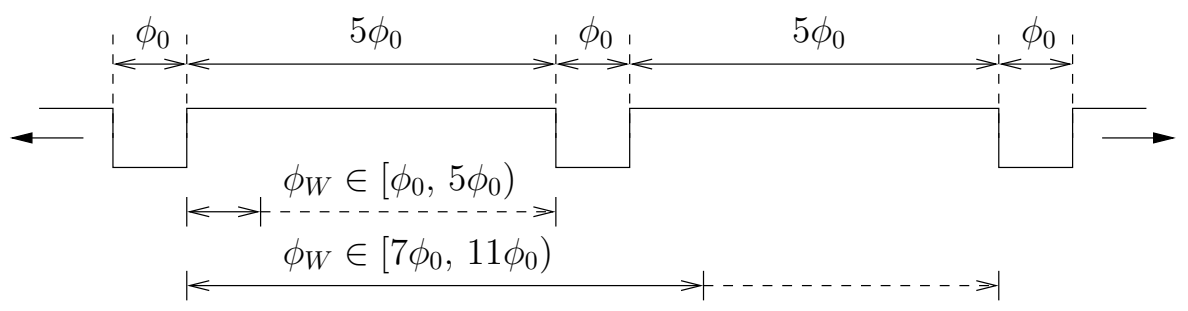

Figure 7: Finding the intervals for the Case A, and for the code 5 .

\begin{tabular}{|c|c|}
\hline & Ranges for $\phi_{W}$ \\
\hline \hline Code 1 & {$\left[3 \phi_{0}, 9 \phi_{0}\right)$} \\
\hline Code 2 & {$\left[\phi_{0}, 2 \phi_{0}\right) \cup\left[4 \phi_{0}, 8 \phi_{0}\right) \cup\left[10 \phi_{0}, 11 \phi_{0}\right)$} \\
\hline Code 3 & {$\left[\phi_{0}, 3 \phi_{0}\right) \cup\left[5 \phi_{0}, 7 \phi_{0}\right) \cup\left[9 \phi_{0}, 11 \phi_{0}\right)$} \\
\hline Code 4 & {$\left[\phi_{0}, 4 \phi_{0}\right) \cup\left[8 \phi_{0}, 11 \phi_{0}\right)$} \\
\hline Code 5 & {$\left[\phi_{0}, 5 \phi_{0}\right) \cup\left[7 \phi_{0}, 11 \phi_{0}\right)$} \\
\hline
\end{tabular}

Table 3: Intervals of the angular window $\phi_{W}$ for the Case A with respect to the codes $(\bigcup$ denotes the union).

\subsubsection{The case $A$}

This case corresponds to the situation leading to the upper bound of the variance, as explained in Section 3.3.2. For each code, it is possible to find values for the angular window for which it is impossible to have $\mathcal{E}_{r} \neq 0$ and $\mathcal{E}_{f} \neq 0$ at the same time. Firstly, we determine the values of the angular window for which this situation occurs. We explain this reasoning with the code 5 since it is easier, and we will generalize the result later for the other codes. To find these values, one has to pick up a fixed value for the angular window, and to move virtually the code from the left to the right, and to check if the entering and leaving angles can both fall into the gaps $\left(\phi_{0}\right)$. Figure 7 illustrates the idea. From this analysis, one can see that, if the angular window $\phi_{W}$ is comprised between $\phi_{0}$ and $5 \phi_{0}$ or between $7 \phi_{0}$ and $11 \phi_{0}$, it is impossible to commit an error on both the entering and leaving angle. These intervals can be generalized for any code $i$, as given in Table 3. The principle is the same, but we have to replace the first 5 by $i$, and the second 5 by $10-i$ (or $N_{1}-i$ ), as a consequence of how to define the different codes

$$
C_{i}=\left[1^{i} 0^{1} 1^{10-i} 0^{1}\right] \quad i=1, \ldots, 5 .
$$

The general case is depicted in Figure 23, and further developed in Section A.3 of the appendix. We can summarize the intervals for the case A, and for all codes in the following compact form

$$
\phi_{W} \bmod \left(12 \phi_{0}\right) \in\left[\phi_{0}, i \phi_{0}\right) \bigcup\left[(i+2) \phi_{0},(10-i) \phi_{0}\right) \bigcup\left[(12-i) \phi_{0}, 11 \phi_{0}\right) \quad i=1, \ldots, 5,
$$

where $\bigcup$ denotes the union.

Now that the intervals have been determined, we are interested in finding the joint $P D F$ of $\mathcal{E}_{r}$ and $\mathcal{E}_{f}$, in order to compute their joint expectation. We can represent the possible joint values of $\mathcal{E}_{r}$ and $\mathcal{E}_{f}$ on a $2 \mathrm{D}$ graphic where the abscissa $\epsilon_{r}$ represents a possible value of $\mathcal{E}_{r}\left(\epsilon_{r} \in\left[0, \phi_{0}\right]\right)$ and the ordinate $\epsilon_{f}$ represents a possible value of $\mathcal{E}_{f}\left(\epsilon_{f} \in\left[-\phi_{0}, 0\right]\right)$. Indeed, this graphic is the support of the joint $P D F$ of $\mathcal{E}_{r}$ and $\mathcal{E}_{f}$. It is represented in Figure 8. The support of this joint $P D F$ is composed of an horizontal line segment from 0 to $\phi_{0}\left(\mathcal{E}_{r} \neq 0\right.$ only), a vertical line segment from $-\phi_{0}$ to $0\left(\mathcal{E}_{f} \neq 0\right.$ only), and a black dot at the origin (no errors). The black dot represents a two dimensional DiRAC $P D F$, and the line segments are uniform $P D F s$, as explained later. There is no part of the support outside the axes which means that is impossible to have $\mathcal{E}_{r} \neq 0$ and $\mathcal{E}_{f} \neq 0$ simultaneously. The line segments are denoted by a black star $(\star)$, and the origin by a black dot $(\bullet)$. 


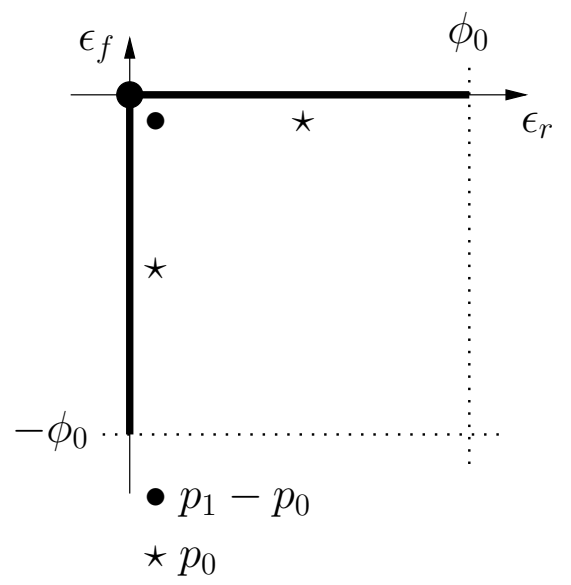

Figure 8: Support of the joint $P D F$ of $\mathcal{E}_{r}$ and $\mathcal{E}_{f}$ for the case A.

Now, we have to associate probabilities and PDFs to these three parts. Assuming time stationarity and as there is no synchronization between the beacons and the receiver, we then assume that the value of $\mathcal{E}_{r}$ is uniformly distributed between 0 and $\phi_{0}$, when $\mathcal{E}_{f}=0$. By symmetry, we assume that the value of $\mathcal{E}_{f}$ is uniformly distributed between $-\phi_{0}$ and 0 , when $\mathcal{E}_{r}=0$. Therefore, the joint $P D F$ of $\mathcal{E}_{r}$ and $\mathcal{E}_{f}$ is given by the following mixture of joint $P D F s$

$$
f_{\mathcal{E}_{r} \mathcal{E}_{f}}\left(\epsilon_{r}, \epsilon_{f}\right)=p \bullet \delta\left(\epsilon_{r}\right) \delta\left(\epsilon_{f}\right)+p^{\star} \delta\left(\epsilon_{r}\right) U_{\left(-\phi_{0}, 0\right)}\left(\epsilon_{f}\right)+p^{\star} \delta\left(\epsilon_{f}\right) U_{\left(0, \phi_{0}\right)}\left(\epsilon_{r}\right),
$$

where:

- $p^{\bullet}$ is the probability to commit no error, and

- $p^{\star}$ is the probability to have $\mathcal{E}_{r} \neq 0$ only. By symmetry, we show that the probability to have $\mathcal{E}_{f} \neq 0$ only is also given by $p^{\star}$, explaining why the notation $p^{\star}$ is the same for both.

The complete explanation about the computation of these probabilities is give in the appendix (Section A.3). Their values are given below

$$
\left\{\begin{array}{l}
p^{\bullet}=p_{1}-p_{0} \\
p^{\star}=p_{0}
\end{array}\right.
$$

We can check that the probabilities for the case A sum up to 1

$$
p^{\bullet}+p^{\star}+p^{\star}=p_{1}-p_{0}+p_{0}+p_{0}=p_{1}+p_{0}=1 .
$$

As another verification, we can also compute the marginal $P D F$ for $\mathcal{E}_{r}$

$$
\begin{aligned}
f_{\mathcal{E}_{r}}\left(\epsilon_{r}\right)= & \int_{-\infty}^{+\infty} f_{\mathcal{E}_{r} \mathcal{E}_{f}}\left(\epsilon_{r}, \epsilon_{f}\right) d \epsilon_{f} \\
= & \left(p_{1}-p_{0}\right) \delta\left(\epsilon_{r}\right) \int_{-\infty}^{+\infty} \delta\left(\epsilon_{f}\right) d \epsilon_{f}+p_{0} \delta\left(\epsilon_{r}\right) \int_{-\infty}^{+\infty} U_{\left(-\phi_{0}, 0\right)}\left(\epsilon_{f}\right) d \epsilon_{f} \\
& +p_{0} U_{\left(0, \phi_{0}\right)}\left(\epsilon_{r}\right) \int_{-\infty}^{+\infty} \delta\left(\epsilon_{f}\right) d \epsilon_{f} \\
= & \left(p_{1}-p_{0}\right) \delta\left(\epsilon_{r}\right)+p_{0} \delta\left(\epsilon_{r}\right)+p_{0} U_{\left(0, \phi_{0}\right)}\left(\epsilon_{r}\right) \\
= & p_{1} \delta\left(\epsilon_{r}\right)+p_{0} U_{\left(0, \phi_{0}\right)}\left(\epsilon_{r}\right)
\end{aligned}
$$


which is the same as equation (24). Likewise, the marginal $P D F$ for the random variable $\mathcal{E}_{f}$ is identical to equation (25). Now that we have the joint $P D F$, we can compute the joint expectation $E\left\{\mathcal{E}_{r}, \mathcal{E}_{f}\right\}$

$$
\begin{aligned}
E\left\{\mathcal{E}_{r}, \mathcal{E}_{f}\right\}= & \iint_{-\infty}^{+\infty} \epsilon_{r} \epsilon_{f} f_{\mathcal{E}_{r} \mathcal{E}_{f}}\left(\epsilon_{r}, \epsilon_{f}\right) d \epsilon_{r} d \epsilon_{f} \\
= & \left(p_{1}-p_{0}\right) \iint_{-\infty}^{+\infty} \epsilon_{r} \epsilon_{f} \delta\left(\epsilon_{r}\right) \delta\left(\epsilon_{f}\right) d \epsilon_{r} d \epsilon_{f} \\
& +p_{0} \iint_{-\infty}^{+\infty} \epsilon_{r} \epsilon_{f} \delta\left(\epsilon_{r}\right) U_{\left(-\phi_{0}, 0\right)}\left(\epsilon_{f}\right) d \epsilon_{r} d \epsilon_{f} \\
& +p_{0} \iint_{-\infty}^{+\infty} \epsilon_{r} \epsilon_{f} \delta\left(\epsilon_{f}\right) U_{\left(0, \phi_{0}\right)}\left(\epsilon_{r}\right) d \epsilon_{r} d \epsilon_{f} \\
= & 0+0+0=0 .
\end{aligned}
$$

The joint expectation is null, meaning that random variables $\mathcal{E}_{r}$ and $\mathcal{E}_{f}$ are orthogonal for the case A. Then, we can compute $C\left\{\Phi_{r}, \Phi_{f}\right\}$ for the case A

$$
C\left\{\Phi_{r}, \Phi_{f}\right\}=C\left\{\mathcal{E}_{r}, \mathcal{E}_{f}\right\}=E\left\{\mathcal{E}_{r}, \mathcal{E}_{f}\right\}-E\left\{\mathcal{E}_{r}\right\} E\left\{\mathcal{E}_{f}\right\}=0-\left(p_{0} \frac{\phi_{0}}{2}\right)\left(-p_{0} \frac{\phi_{0}}{2}\right)=p_{0}^{2} \frac{\phi_{0}^{2}}{4}
$$

and, finally, we can compute the variance of $\Phi_{b}$ for the case A

$$
\begin{aligned}
\sigma_{\Phi_{b}}^{2} & =\frac{\sigma_{\mathcal{E}_{r}}^{2}+C\left\{\mathcal{E}_{r}, \mathcal{E}_{f}\right\}}{2} \\
& =\frac{\left(p_{0} \frac{\phi_{0}^{2}}{3}-p_{0}^{2} \frac{\phi_{0}^{2}}{4}\right)+\left(p_{0}^{2} \frac{\phi_{0}^{2}}{4}\right)}{2} \\
& =p_{0} \frac{\phi_{0}^{2}}{6} .
\end{aligned}
$$

Note that this value corresponds to the upper bound defined in Section 3.3.2. As long as the angular window $\phi_{W}$ belongs to admissible ranges for the case $A$, the variance of $\Phi_{b}$ does not depend on the angular window.

\subsubsection{The case 0}

The case 0 arises when the angular window $\phi_{W}$ is comprised between 0 and $\phi_{0}$, for all codes. This case is similar to the case $\mathrm{A}$ in that it is impossible to have $\mathcal{E}_{r} \neq 0$ and $\mathcal{E}_{f} \neq 0$ simultaneously. But, unlike the case $\mathrm{A}$, the intervals for the committed errors range from 0 to $\phi_{W}$ in absolute value

$$
\begin{aligned}
0 & \leq \mathcal{E}_{r} \leq \phi_{W}, \\
-\phi_{W} & \leq \mathcal{E}_{f} \leq 0 .
\end{aligned}
$$

Furthermore, there are parts where there is no detection at all, when the angular window is entirely comprised in a zero symbol duration. These comments show that the probabilities depend on the angular window. Since we have $\phi_{W} \in\left[0, \phi_{0}\right)$ for the case 0 , let us introduce a parameter to express $\phi_{W}$ in function of $\phi_{0}$

$$
\phi_{W}=\lambda_{0} \phi_{0}, \quad \lambda_{0} \in[0,1) .
$$

The support of the joint $P D F$ is represented in Figure 9. The support of this joint $P D F$ is composed of an horizontal line segment from 0 to $\lambda_{0} \phi_{0}\left(\mathcal{E}_{r} \neq 0\right.$ only), a vertical line segment from 0 to $-\lambda_{0} \phi_{0}\left(\mathcal{E}_{f} \neq 0\right.$ only), and a black dot at the origin (no errors). The black dot represents a two dimensional DIRAC PDF, and the line segments are uniform PDFs, as explained hereafter. 


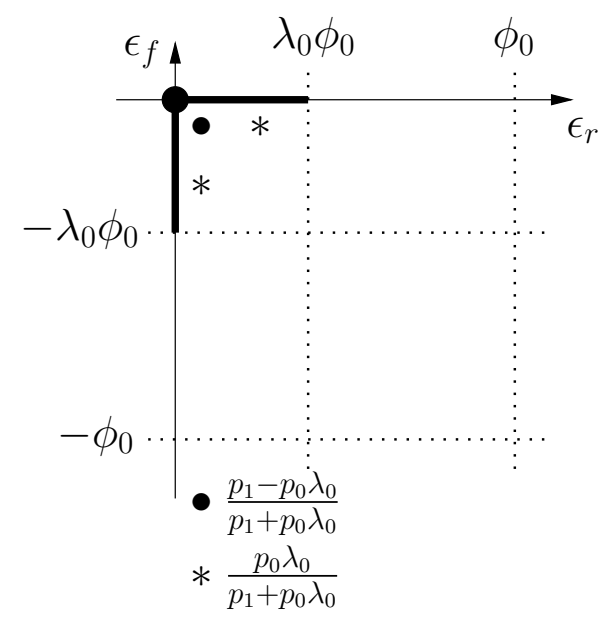

Figure 9: Support of the joint $P D F$ of $\mathcal{E}_{r}$ and $\mathcal{E}_{f}$ for the case 0.

There is no part of the support outside the axes which means that is impossible to have $\mathcal{E}_{r} \neq 0$ and $\mathcal{E}_{f} \neq 0$ simultaneously. The line segments are denoted by an asterisk (*), and the origin by a black dot $(\bullet)$.

Now, we have to associate probabilities and PDFs to these three parts. Assuming time stationarity and, as there is no synchronization between the beacons and the receiver, we then assume that the value of $\mathcal{E}_{r}$ is uniformly distributed between 0 and $\lambda_{0} \phi_{0}$, when $\mathcal{E}_{f}=0$. By symmetry, we assume that the value of $\mathcal{E}_{f}$ is uniformly distributed between $-\lambda_{0} \phi_{0}$ and 0 , when $\mathcal{E}_{r}=0$. Therefore, the joint $P D F$ of $\mathcal{E}_{r}$ and $\mathcal{E}_{f}$ is given by the following mixture of joint $P D F s$

$$
f_{\mathcal{E}_{r} \mathcal{E}_{f}}\left(\epsilon_{r}, \epsilon_{f}\right)=p^{\bullet} \delta\left(\epsilon_{r}\right) \delta\left(\epsilon_{f}\right)+p^{*} \delta\left(\epsilon_{r}\right) U_{\left(-\lambda_{0} \phi_{0}, 0\right)}\left(\epsilon_{f}\right)+p^{*} \delta\left(\epsilon_{f}\right) U_{\left(0, \lambda_{0} \phi_{0}\right)}\left(\epsilon_{r}\right)
$$

where:

- $p^{\bullet}$ is the probability to commit no error, and

- $p^{*}$ is the probability to have $\mathcal{E}_{r} \neq 0$ only. By symmetry, we show that the probability to have $\mathcal{E}_{f} \neq 0$ only is also given by $p^{*}$, explaining why the notation $p^{*}$ is the same for both.

The complete explanation about the computation of these probabilities is given in the appendix (Section A.4). Their values are

$$
\left\{\begin{array}{l}
p^{\bullet}=\frac{p_{1}-p_{0} \lambda_{0}}{p_{1}+p_{0} \lambda_{0}} \\
p^{*}=\frac{p_{0} \lambda_{0}}{p_{1}+p_{0} \lambda_{0}}
\end{array}\right.
$$

We can check that the probabilities for the case 0 sum up to 1

$$
p^{\bullet}+p^{*}+p^{*}=\frac{p_{1}-p_{0} \lambda_{0}}{p_{1}+p_{0} \lambda_{0}}+2 \frac{p_{0} \lambda_{0}}{p_{1}+p_{0} \lambda_{0}}=\frac{p_{1}+p_{0} \lambda_{0}}{p_{1}+p_{0} \lambda_{0}}=1
$$

Like for the case A, the joint $P D F$ of $\mathcal{E}_{r}$ and $\mathcal{E}_{f}$, as well as the support of the joint $P D F$ (Figure 9), show that $\mathcal{E}_{r}$ and $\mathcal{E}_{f}$ are orthogonal (see appendix A.4 for the details)

$$
E\left\{\mathcal{E}_{r}, \mathcal{E}_{f}\right\}=\iint_{-\infty}^{+\infty} \epsilon_{r} \epsilon_{f} f_{\mathcal{E}_{r} \mathcal{E}_{f}}\left(\epsilon_{r}, \epsilon_{f}\right) d \epsilon_{r} d \epsilon_{f}=0
$$

But, unlike the case A (and all other cases), the marginal $P D F$ of $\mathcal{E}_{r}$ and $\mathcal{E}_{f}$ have changed, as well as their expectations and variances. The marginal $P D F$ of $\mathcal{E}_{r}$ is given by (see appendix A.4 for 
more details)

$$
\begin{aligned}
f_{\mathcal{E}_{r}}\left(\epsilon_{r}\right) & =\int_{-\infty}^{+\infty} f_{\mathcal{E}_{r} \mathcal{E}_{f}}\left(\epsilon_{r}, \epsilon_{f}\right) d \epsilon_{f} \\
& =\frac{p_{1}}{p_{1}+p_{0} \lambda_{0}} \delta\left(\epsilon_{r}\right)+\frac{p_{0} \lambda_{0}}{p_{1}+p_{0} \lambda_{0}} U_{\left(0, \lambda_{0} \phi_{0}\right)}\left(\epsilon_{r}\right)
\end{aligned}
$$

The marginal $P D F$ of $\mathcal{E}_{f}$ is given by

$$
f_{\mathcal{E}_{f}}\left(\epsilon_{f}\right)=\frac{p_{1}}{p_{1}+p_{0} \lambda_{0}} \delta\left(\epsilon_{f}\right)+\frac{p_{0} \lambda_{0}}{p_{1}+p_{0} \lambda_{0}} U_{\left(-\lambda_{0} \phi_{0}, 0\right)}\left(\epsilon_{f}\right) .
$$

Their expectations are given by, using result 123,

$$
E\left\{\mathcal{E}_{r}\right\}=\frac{p_{0} \lambda_{0}}{\left(p_{1}+p_{0} \lambda_{0}\right)} \frac{\lambda_{0} \phi_{0}}{2}=-E\left\{\mathcal{E}_{f}\right\}
$$

and their variances are given by, using result 125 ,

$$
\operatorname{var}\left\{\mathcal{E}_{r}\right\}=\operatorname{var}\left\{\mathcal{E}_{f}\right\}=\frac{\left(\lambda_{0} \phi_{0}\right)^{2}}{12} \frac{p_{0} \lambda_{0}\left(4 p_{1}+p_{0} \lambda_{0}\right)}{\left(p_{1}+p_{0} \lambda_{0}\right)^{2}} .
$$

Then, we can compute $C\left\{\mathcal{E}_{r}, \mathcal{E}_{f}\right\}$ for the case 0

$$
C\left\{\mathcal{E}_{r}, \mathcal{E}_{f}\right\}=E\left\{\mathcal{E}_{r}, \mathcal{E}_{f}\right\}-E\left\{\mathcal{E}_{r}\right\} E\left\{\mathcal{E}_{f}\right\}=\left(\frac{p_{0} \lambda_{0}}{\left(p_{1}+p_{0} \lambda_{0}\right)} \frac{\lambda_{0} \phi_{0}}{2}\right)^{2}=\frac{\left(p_{0} \lambda_{0}\right)^{2}}{\left(p_{1}+p_{0} \lambda_{0}\right)^{2}} \frac{\left(\lambda_{0} \phi_{0}\right)^{2}}{4}
$$

and, finally, we can obtain the variance of $\Phi_{b}$ for the case 0

$$
\begin{aligned}
\sigma_{\Phi_{b}}^{2} & =\frac{\sigma_{\mathcal{E}_{r}}^{2}+C\left\{\mathcal{E}_{r}, \mathcal{E}_{f}\right\}}{2} \\
& =p_{0} \frac{\phi_{0}^{2}}{6} \frac{\lambda_{0}^{3}}{\left(p_{1}+p_{0} \lambda_{0}\right)} \\
& =p_{0} \frac{\phi_{0}^{2}}{6} P_{0}\left(\lambda_{0}\right),
\end{aligned}
$$

where the first part is the same as for the case A, and the second part is a function (polynomial ratio) of the parameter $\lambda_{0}$

$$
P_{0}\left(\lambda_{0}\right)=\frac{\lambda_{0}^{3}}{p_{1}+p_{0} \lambda_{0}}
$$

Note that $P_{0}\left(\lambda_{0}\right)$ satisfies

$$
0 \leq P_{0}\left(\lambda_{0}\right)<1 \quad \text { for } \lambda_{0} \in[0,1)
$$

This function is equal to 0 when $\lambda_{0}=0$ (angular window reduced to 0 ), monotonically increases with $\lambda_{0}$, and is equal to 1 when $\lambda_{0}=1$. Note that the case 0 represents a particular situation. Indeed, the decoded signal is composed of one rising edge, and one falling edge only. It means that there is not enough information to compute the beacon's ID, even if an angle could be computed for this signal. The hardware then discards this information, so that the high level positioning algorithm does not even need to deal with this situation. This case appears when the received power is sufficiently low, or equivalently when the distance is high enough. Therefore, this case could seem uninteresting, but we study this case in order to be comprehensive in our study of the variance w.r.t. the angular window. Moreover, we could consider sending this angle to the high level positioning algorithm if it can deal with unidentified beacons. 


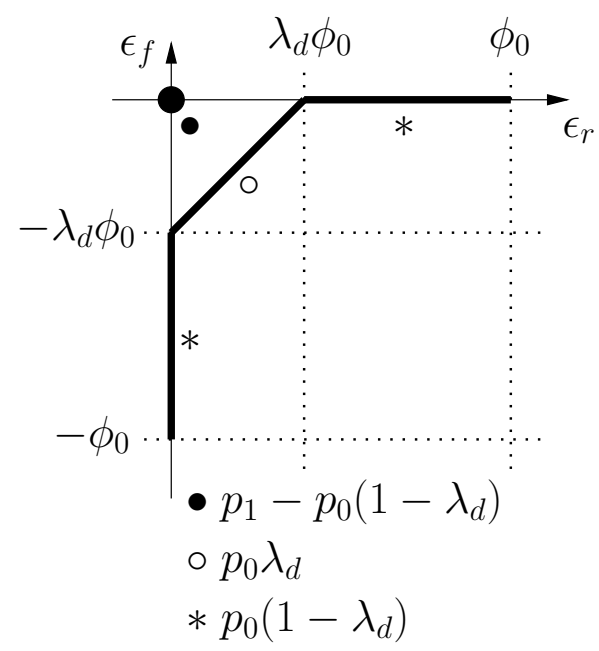

Figure 10: Support of the joint $P D F$ of $\mathcal{E}_{r}$ and $\mathcal{E}_{f}$ for the case D.

\subsubsection{The case $D$}

In that case, it is possible to have $\mathcal{E}_{r} \neq 0$ and $\mathcal{E}_{f} \neq 0$ simultaneously. When this occurs, it can be shown that their difference is constant (see Figure 25, lines 4 and 8, or red parts, in the appendix A.5). This situation appears for values of the angular window such that

$$
\phi_{W} \bmod \left(12 \phi_{0}\right) \in\left[11 \phi_{0}, 12 \phi_{0}\right) .
$$

However, this constant difference depends on the particular value of the angular window $\left(\epsilon_{r}-\epsilon_{f}=\right.$ $\lambda_{d} \phi_{0}$, where $\lambda_{d}$ is defined hereafter). Therefore, the support of the joint $P D F$ depends on the value of the angular window, and as a consequence, we introduce a parameter to express $\phi_{W}$ in that interval

$$
\phi_{W} \bmod \left(12 \phi_{0}\right)=11 \phi_{0}+\lambda_{d} \phi_{0}, \quad \lambda_{d} \in[0,1) .
$$

The support of the joint $P D F$ is represented in Figure 10. It is composed of an horizontal line segment from $\lambda_{d} \phi_{0}$ to $\phi_{0}\left(\mathcal{E}_{r} \neq 0\right.$ only), a vertical line segment from $-\phi_{0}$ to $-\lambda_{d} \phi_{0}\left(\mathcal{E}_{f} \neq 0\right.$ only), a slanted segment $\left(\mathcal{E}_{r} \neq 0\right.$ and $\left.\mathcal{E}_{f} \neq 0\right)$ joining the previous ones, and a black dot at the origin (no errors). The black dot represents a two dimensional DiRAC PDF, and the line segments are uniform PDFs, as explained hereafter. The horizontal and vertical line segments are denoted by an asterisk $(*)$, the slanted line segment is denoted by a circle (o) and the origin by a black dot $(\bullet)$.

Now, we have to associate probabilities and PDFs to these four parts. Again, we assume the time stationarity, and we assume that the pairs of errors are uniformly distributed along the line segments. Therefore, the joint $P D F$ of $\mathcal{E}_{r}$ and $\mathcal{E}_{f}$ is given by the following mixture of joint PDFs

$$
\begin{aligned}
f_{\mathcal{E}_{r} \mathcal{E}_{f}}\left(\epsilon_{r}, \epsilon_{f}\right) & =p^{\bullet} \delta\left(\epsilon_{r}\right) \delta\left(\epsilon_{f}\right) \\
& +p^{*} \delta\left(\epsilon_{r}\right) U_{\left(-\phi_{0}, \lambda_{d} \phi_{0}\right)}\left(\epsilon_{f}\right) \\
& +p^{*} \delta\left(\epsilon_{f}\right) U_{\left(\lambda_{d} \phi_{0}, \phi_{0}\right)}\left(\epsilon_{r}\right) \\
& +p^{\circ} 2 \delta\left(\left(\epsilon_{r}-\epsilon_{f}\right)-\lambda_{d} \phi_{0}\right) U_{\left(-\lambda_{d} \phi_{0}, \lambda_{d} \phi_{0}\right)}\left(\epsilon_{r}+\epsilon_{f}\right)
\end{aligned}
$$

where:

- $p^{\bullet}$ is the probability to commit no error,

- $p^{*}$ is the probability to have $\mathcal{E}_{r} \neq 0$ only. By symmetry, we show that the probability to have $\mathcal{E}_{f} \neq 0$ only is also given by $p^{*}$, explaining why the notation $p^{*}$ is the same for both, and 
- $p^{\circ}$ is the probability to have $\mathcal{E}_{r} \neq 0$ and $\mathcal{E}_{f} \neq 0$.

The complete explanation about the computation of these probabilities is provided in Section A.5 of the appendix. Their values are given below

$$
\left\{\begin{array}{l}
p^{\bullet}=p_{1}-p_{0}\left(1-\lambda_{d}\right) \\
p^{\circ}=p_{0} \lambda_{d} \\
p^{*}=p_{0}\left(1-\lambda_{d}\right) .
\end{array}\right.
$$

We can check that the probabilities for the case D sum up to 1

$$
p^{\bullet}+p^{\circ}+2 p^{*}=p_{1}-p_{0}\left(1-\lambda_{d}\right)+p_{0} \lambda_{d}+2 p_{0}\left(1-\lambda_{d}\right)=p_{1}+p_{0}=1 .
$$

The joint expectation is (see appendix A.5)

$$
E\left\{\mathcal{E}_{r}, \mathcal{E}_{f}\right\}=-p_{0} \lambda_{d}^{3} \frac{\phi_{0}^{2}}{6} .
$$

Then, we can compute $C\left\{\mathcal{E}_{r}, \mathcal{E}_{f}\right\}$ for the case $\mathrm{D}$

$$
C\left\{\mathcal{E}_{r}, \mathcal{E}_{f}\right\}=E\left\{\mathcal{E}_{r}, \mathcal{E}_{f}\right\}-E\left\{\mathcal{E}_{r}\right\} E\left\{\mathcal{E}_{f}\right\}=-p_{0} \lambda_{d}^{3} \frac{\phi_{0}^{2}}{6}+p_{0}^{2} \frac{\phi_{0}^{2}}{4},
$$

and, finally, we can obtain the variance of $\Phi_{b}$ for the case D

$$
\begin{aligned}
\sigma_{\Phi_{b}}^{2} & =\frac{\sigma_{\mathcal{E}_{r}}^{2}+C\left\{\mathcal{E}_{r}, \mathcal{E}_{f}\right\}}{2} \\
& =p_{0} \frac{\phi_{0}^{2}}{6} P_{D}\left(\lambda_{d}\right),
\end{aligned}
$$

where the first part is the same as for the case A, and the second part is a polynomial function of the parameter $\lambda_{d}$

$$
P_{D}\left(\lambda_{d}\right)=1-\frac{\lambda_{d}^{3}}{2}
$$

Note that, again, $P_{D}\left(\lambda_{d}\right)$ is inferior to 1.

\subsubsection{The case $\mathrm{E}$}

In that case, it is also possible to have $\mathcal{E}_{r} \neq 0$ and $\mathcal{E}_{f} \neq 0$ simultaneously. When it happens, it can be shown that their difference is constant (see Figure 26, lines 3 and 7 , or red parts, in the appendix A.6). It appears for values of the angular window such that

$$
\phi_{W} \bmod \left(12 \phi_{0}\right) \in\left[0, \phi_{0}\right), \quad \phi_{W} \geq 12 \phi_{0} .
$$

However, this constant difference depends on the particular value of the angular window $\left(\epsilon_{r}-\epsilon_{f}=\right.$ $\left(1+\lambda_{e}\right) \phi_{0}$, where $\lambda_{e}$ is defined hereafter). Therefore, the support of the joint PDF depends on the value of the angular window, and as a consequence, we introduce a parameter to express $\phi_{W}$ in that interval

$$
\phi_{W} \bmod \left(12 \phi_{0}\right)=12 \phi_{0}+\lambda_{e} \phi_{0}, \quad \lambda_{e} \in[0,1) .
$$

The support of the joint $P D F$ is represented in Figure 11. It is composed of an horizontal line segment from 0 to $\lambda_{e} \phi_{0}\left(\mathcal{E}_{r} \neq 0\right.$ only), a vertical line segment from $-\lambda_{e} \phi_{0}$ to 0 ( $\mathcal{E}_{f} \neq 0$ only), a slanted segment $\left(\mathcal{E}_{r} \neq 0\right.$ and $\left.\mathcal{E}_{f} \neq 0\right)$, and a black dot at the origin (no errors). The black dot represents a two dimensional DIRAC PDF, and the line segments are uniform PDFs, as explained hereafter. The horizontal and vertical line segments are denoted by an asterisk $(*)$, the slanted line segment is denoted by a circle (०) and the origin by a black dot $(\bullet)$. 


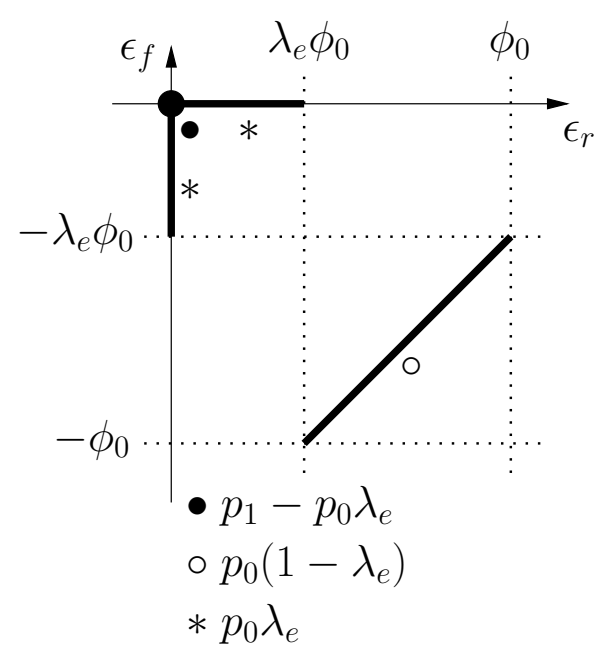

Figure 11: Support of the joint $P D F$ of $\mathcal{E}_{r}$ and $\mathcal{E}_{f}$ for the case E.

Now, we have to associate probabilities and PDFs to these four parts. Again, we assume the time stationarity, and we assume that the pairs of errors are uniformly distributed along the line segments. Therefore, the joint $P D F$ of $\mathcal{E}_{r}$ and $\mathcal{E}_{f}$ is given by the following mixture of joint PDFs

$$
\begin{aligned}
f_{\mathcal{E}_{r} \mathcal{E}_{f}}\left(\epsilon_{r}, \epsilon_{f}\right) & =p^{\bullet} \delta\left(\epsilon_{r}\right) \delta\left(\epsilon_{f}\right) \\
& +p^{*} \delta\left(\epsilon_{r}\right) U_{\left(-\lambda_{e} \phi_{0}, 0\right)}\left(\epsilon_{f}\right) \\
& +p^{*} \delta\left(\epsilon_{f}\right) U_{\left(0, \lambda_{e} \phi_{0}\right)}\left(\epsilon_{r}\right) \\
& +p^{\circ} 2 \delta\left(\left(\epsilon_{r}-\epsilon_{f}\right)-\left(1+\lambda_{e}\right) \phi_{0}\right) U_{\left(-\left(1-\lambda_{e}\right) \phi_{0},\left(1-\lambda_{e}\right) \phi_{0}\right)}\left(\epsilon_{r}+\epsilon_{f}\right),
\end{aligned}
$$

where:

- $p^{\bullet}$ is the probability to commit no error,

- $p^{*}$ is the probability to have $\mathcal{E}_{r} \neq 0$ only. By symmetry, we show that the probability to have $\mathcal{E}_{f} \neq 0$ only is also given by $p^{*}$, explaining why the notation $p^{*}$ is the same for both, and

- $p^{\circ}$ is the probability to have $\mathcal{E}_{r} \neq 0$ and $\mathcal{E}_{f} \neq 0$.

The complete explanation about the computation of these probabilities is given in the appendix (Section A.6). Their values are

$$
\left\{\begin{array}{l}
p^{\bullet}=p_{1}-p_{0} \lambda_{e} \\
p^{\circ}=p_{0}\left(1-\lambda_{e}\right) \\
p^{*}=p_{0} \lambda_{e} .
\end{array}\right.
$$

We can check that the probabilities for the case E sum up to 1

$$
p^{\bullet}+p^{\circ}+2 p^{*}=p_{1}-p_{0} \lambda_{e}+p_{0}\left(1-\lambda_{e}\right)+2 p_{0} \lambda_{e}=p_{1}+p_{0}=1 .
$$

The computation of the joint expectation gives (see appendix A.6)

$$
E\left\{\mathcal{E}_{r}, \mathcal{E}_{f}\right\}=-p_{0} \frac{\phi_{0}^{2}}{6}\left(1-\lambda_{e}\right)\left(1+4 \lambda_{e}+\lambda_{e}^{2}\right) .
$$

Then, we can compute $C\left\{\mathcal{E}_{r}, \mathcal{E}_{f}\right\}$ for the case $\mathrm{E}$

$$
C\left\{\mathcal{E}_{r}, \mathcal{E}_{f}\right\}=E\left\{\mathcal{E}_{r}, \mathcal{E}_{f}\right\}-E\left\{\mathcal{E}_{r}\right\} E\left\{\mathcal{E}_{f}\right\}=-p_{0} \frac{\phi_{0}^{2}}{6}\left(1-\lambda_{e}\right)\left(1+4 \lambda_{e}+\lambda_{e}^{2}\right)+p_{0}^{2} \frac{\phi_{0}^{2}}{4},
$$



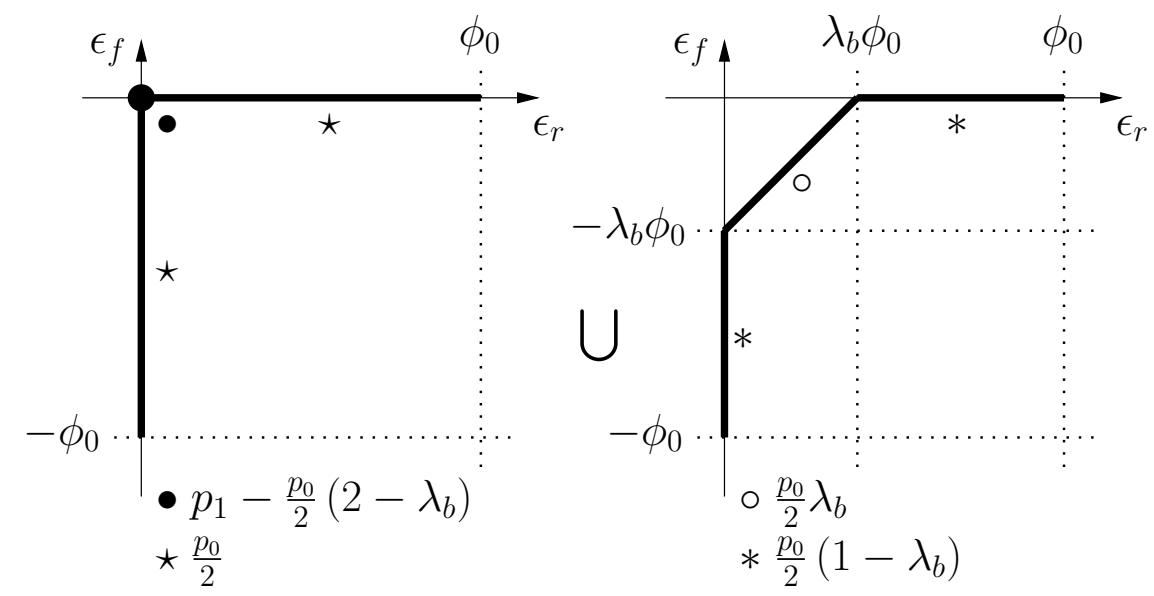

Figure 12: Support of the joint $P D F$ of $\mathcal{E}_{r}$ and $\mathcal{E}_{f}$ for the case B.

and, finally, we can obtain the variance of $\Phi_{b}$ for the case $E$

$$
\begin{aligned}
\sigma_{\Phi_{b}}^{2} & =\frac{\sigma_{\mathcal{E}_{r}}^{2}+C\left\{\mathcal{E}_{r}, \mathcal{E}_{f}\right\}}{2} \\
& =p_{0} \frac{\phi_{0}^{2}}{6} P_{E}\left(\lambda_{e}\right)
\end{aligned}
$$

where the first part is the same as for the case A, and the second part is a polynomial function of the parameter $\lambda_{e}$

$$
P_{E}\left(\lambda_{e}\right)=\frac{1-3 \lambda_{e}+3 \lambda_{e}^{2}+\lambda_{e}^{3}}{2}
$$

\subsubsection{The case $B$}

When analyzing the possible pairs of values $\left(\mathcal{E}_{r}, \mathcal{E}_{f}\right)$ (see Figure 27 in the appendix A.7), it appears that the case $\mathrm{B}$ is a combination of the case $\mathrm{A}$ and the case $\mathrm{D}$. This case occurs for values of the angular window such that

$$
\phi_{W} \bmod \left(12 \phi_{0}\right) \in\left[i \phi_{0},(i+1) \phi_{0}\right) \bigcup\left[(10-i) \phi_{0},(11-i) \phi_{0}\right), \quad i=1,2,3,4 .
$$

Note that this case does not occur for the code 5, as a consequence of its particular shape. Like for the case $\mathrm{D}$, the support of the joint $P D F$ depends on the value of the angular window, and as a consequence, we introduce a parameter $\lambda_{b}$ to have an analytical expression of $\phi_{W}$

$$
\phi_{W} \bmod \left(12 \phi_{0}\right)=i \phi_{0}+\lambda_{b} \phi_{0}, \quad \lambda_{b} \in[0,1), i=1,2,3,4
$$

in the first part of the interval, or

$$
\phi_{W} \bmod \left(12 \phi_{0}\right)=(10-i) \phi_{0}+\lambda_{b} \phi_{0}, \quad \lambda_{b} \in[0,1), i=1,2,3,4,
$$

in the second part of the interval. The support of the joint PDF is represented in Figure 12. Indeed, the support of the joint $P D F$ is obtained by the superimposition of both parts. However, we chose to represent this support with separate parts for two reasons: 1) we want that each line segment represents a uniform $P D F$, and 2) we can use all the previous results about the cases that we have already studied. The joint $P D F$ of $\mathcal{E}_{r}$ and $\mathcal{E}_{f}$ is given by the following mixture of 
joint PDFs

$$
\begin{aligned}
f_{\mathcal{E}_{r} \mathcal{E}_{f}}\left(\epsilon_{r}, \epsilon_{f}\right) & =p^{\bullet} \delta\left(\epsilon_{r}\right) \delta\left(\epsilon_{f}\right) \\
& +p^{\star} \delta\left(\epsilon_{r}\right) U_{\left(-\phi_{0}, 0\right)}\left(\epsilon_{f}\right) \\
& +p^{\star} \delta\left(\epsilon_{f}\right) U_{\left(0, \phi_{0}\right)}\left(\epsilon_{r}\right) \\
& +p^{*} \delta\left(\epsilon_{r}\right) U_{\left(-\phi_{0}, \lambda_{b} \phi_{0}\right)}\left(\epsilon_{f}\right) \\
& +p^{*} \delta\left(\epsilon_{f}\right) U_{\left(\lambda_{b} \phi_{0}, \phi_{0}\right)}\left(\epsilon_{r}\right) \\
& +p^{\circ} 2 \delta\left(\left(\epsilon_{r}-\epsilon_{f}\right)-\lambda_{b} \phi_{0}\right) U_{\left(-\lambda_{b} \phi_{0}, \lambda_{b} \phi_{0}\right)}\left(\epsilon_{r}+\epsilon_{f}\right)
\end{aligned}
$$

where the different probabilities have the same meaning as previously defined. The complete explanation about the computation of these probabilities is given in the appendix (Section A.7). Their values are

$$
\left\{\begin{array}{l}
p^{\bullet}=p_{1}-\frac{p_{0}}{2}\left(2-\lambda_{b}\right) \\
p^{\circ}=\frac{p_{0}}{2} \lambda_{b} \\
p^{\star}=\frac{p_{0}}{2} \\
p^{*}=\frac{p_{0}}{2}\left(1-\lambda_{b}\right) .
\end{array}\right.
$$

We can check that the probabilities for the case B sum up to 1

$$
p^{\bullet}+p^{\circ}+2 p^{\star}+2 p^{*}=p_{1}-\frac{p_{0}}{2}\left(2-\lambda_{b}\right)+\frac{p_{0}}{2} \lambda_{b}+2 \frac{p_{0}}{2}+2 \frac{p_{0}}{2}\left(1-\lambda_{b}\right)=p_{1}+p_{0}=1 .
$$

The computation of the joint expectation gives (see appendix A.7)

$$
E\left\{\mathcal{E}_{r}, \mathcal{E}_{f}\right\}=-p_{0} \lambda_{b}^{3} \frac{\phi_{0}^{2}}{12} .
$$

Then, we can compute $C\left\{\mathcal{E}_{r}, \mathcal{E}_{f}\right\}$ for the case $\mathrm{B}$

$$
C\left\{\mathcal{E}_{r}, \mathcal{E}_{f}\right\}=E\left\{\mathcal{E}_{r}, \mathcal{E}_{f}\right\}-E\left\{\mathcal{E}_{r}\right\} E\left\{\mathcal{E}_{f}\right\}=-p_{0} \lambda_{b}^{3} \frac{\phi_{0}^{2}}{12}+p_{0}^{2} \frac{\phi_{0}^{2}}{4},
$$

and, finally, we can obtain the variance of $\Phi_{b}$ for the case B

$$
\begin{aligned}
\sigma_{\Phi_{b}}^{2} & =\frac{\sigma_{\mathcal{E}_{r}}^{2}+C\left\{\mathcal{E}_{r}, \mathcal{E}_{f}\right\}}{2} \\
& =p_{0} \frac{\phi_{0}^{2}}{6} P_{B}\left(\lambda_{b}\right),
\end{aligned}
$$

where the first part is the same as for the case A, and the second part is a polynomial function of the parameter $\lambda_{b}$

$$
P_{B}\left(\lambda_{b}\right)=1-\frac{\lambda_{b}^{3}}{4}
$$

\subsubsection{The case $\mathrm{C}$}

When analyzing the possible pairs of values for $\left(\mathcal{E}_{r}, \mathcal{E}_{f}\right)$ (see Figure 28 in the appendix A.8), it appears that the case $\mathrm{C}$ is a combination of the case $\mathrm{A}$ and the case $\mathrm{E}$. This happens for values of the angular window such that

$$
\phi_{W} \bmod \left(12 \phi_{0}\right) \in\left[(i+1) \phi_{0},(i+2) \phi_{0}\right) \bigcup\left[(11-i) \phi_{0},(12-i) \phi_{0}\right), \quad i=1,2,3,4 .
$$

Note that this case does not occur for the code 5 , as a consequence of its particular shape. Like for the case E, the support of the joint $P D F$ depends on the value of the angular window, and as a consequence, we introduce a parameter to express $\phi_{W}$ in terms of $\phi_{0}$

$$
\phi_{W} \bmod \left(12 \phi_{0}\right)=(i+1) \phi_{0}+\lambda_{c} \phi_{0}, \quad \lambda_{c} \in[0,1), i=1,2,3,4,
$$



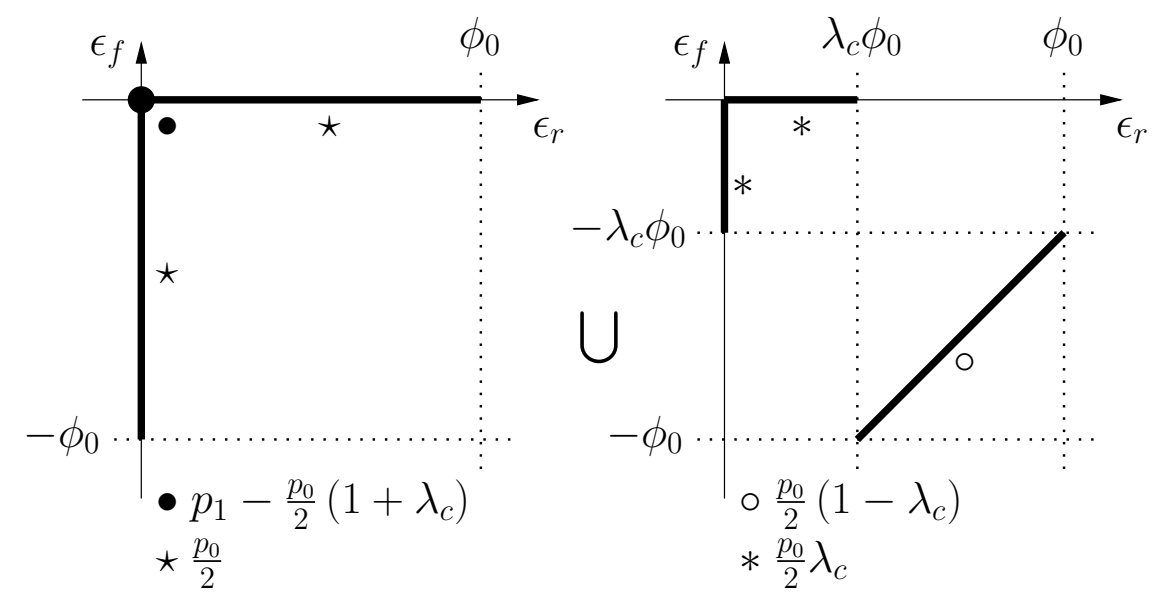

Figure 13: Support of the joint $P D F$ of $\mathcal{E}_{r}$ and $\mathcal{E}_{f}$ for the case C.

in the first part of the interval, or

$$
\phi_{W} \bmod \left(12 \phi_{0}\right)=(11-i) \phi_{0}+\lambda_{c} \phi_{0}, \quad \lambda_{c} \in[0,1), i=1,2,3,4,
$$

in the second part of the interval. The support of the joint $P D F$ is represented in Figure 13. Indeed, the support of the joint $P D F$ is obtained by the superimposition of both parts. However, we chose to represent this support with separate parts for the same reasons as for the case $\mathrm{B}$. The joint $P D F$ of $\mathcal{E}_{r}$ and $\mathcal{E}_{f}$ is given by the following mixture of joint $P D F s$

$$
\begin{aligned}
f_{\mathcal{E}_{r} \mathcal{E}_{f}}\left(\epsilon_{r}, \epsilon_{f}\right) & =p^{\bullet} \delta\left(\epsilon_{r}\right) \delta\left(\epsilon_{f}\right) \\
& +p^{\star} \delta\left(\epsilon_{r}\right) U_{\left(-\phi_{0}, 0\right)}\left(\epsilon_{f}\right) \\
& +p^{\star} \delta\left(\epsilon_{f}\right) U_{\left(0, \phi_{0}\right)}\left(\epsilon_{r}\right) \\
& +p^{*} \delta\left(\epsilon_{r}\right) U_{\left(-\lambda_{c} \phi_{0}, 0\right)}\left(\epsilon_{f}\right) \\
& +p^{*} \delta\left(\epsilon_{f}\right) U_{\left(0, \lambda_{c} \phi_{0}\right)}\left(\epsilon_{r}\right) \\
& +p^{\circ} 2 \delta\left(\left(\epsilon_{r}-\epsilon_{f}\right)-\left(1+\lambda_{c}\right) \phi_{0}\right) U_{\left(-\left(1-\lambda_{c}\right) \phi_{0},\left(1-\lambda_{c}\right) \phi_{0}\right)}\left(\epsilon_{r}+\epsilon_{f}\right) .
\end{aligned}
$$

where the different probabilities have the same meaning as previously defined. The complete explanation about the computation of these probabilities is given in the appendix (Section A.8). Their values are

$$
\left\{\begin{array}{l}
p^{\bullet}=p_{1}-\frac{p_{0}}{2}\left(1+\lambda_{c}\right) \\
p^{\circ}=\frac{p_{0}}{2}\left(1-\lambda_{c}\right) \\
p^{\star}=\frac{p_{0}}{2} \\
p^{*}=\frac{p_{0}}{2} \lambda_{c} .
\end{array}\right.
$$

We can check that the probabilities for the case $\mathrm{C}$ sum up to 1

$$
p^{\bullet}+p^{\circ}+2 p^{\star}+2 p^{*}=p_{1}-\frac{p_{0}}{2}\left(1+\lambda_{c}\right)+\frac{p_{0}}{2}\left(1-\lambda_{c}\right)+2 \frac{p_{0}}{2}+2 \frac{p_{0}}{2} \lambda_{c}=p_{1}+p_{0}=1 .
$$

The computation of the joint expectation gives (see appendix A.8)

$$
E\left\{\mathcal{E}_{r}, \mathcal{E}_{f}\right\}=-p_{0} \frac{\phi_{0}^{2}}{6} \frac{\left(1-\lambda_{c}\right)\left(1+4 \lambda_{c}+\lambda_{c}^{2}\right)}{2} .
$$

Then, we can compute $C\left\{\mathcal{E}_{r}, \mathcal{E}_{f}\right\}$ for the case $\mathrm{C}$

$$
C\left\{\mathcal{E}_{r}, \mathcal{E}_{f}\right\}=E\left\{\mathcal{E}_{r}, \mathcal{E}_{f}\right\}-E\left\{\mathcal{E}_{r}\right\} E\left\{\mathcal{E}_{f}\right\}=-p_{0} \frac{\phi_{0}^{2}}{6} \frac{\left(1-\lambda_{c}\right)\left(1+4 \lambda_{c}+\lambda_{c}^{2}\right)}{2}+p_{0}^{2} \frac{\phi_{0}^{2}}{4}
$$


and, finally, we can obtain the variance of $\Phi_{b}$ for the case C

$$
\begin{aligned}
\sigma_{\Phi_{b}}^{2} & =\frac{\sigma_{\mathcal{E}_{r}}^{2}+C\left\{\mathcal{E}_{r}, \mathcal{E}_{f}\right\}}{2} \\
& =p_{0} \frac{\phi_{0}^{2}}{6} P_{C}\left(\lambda_{c}\right),
\end{aligned}
$$

where the first part is the same as for the case A, and the second part is a polynomial function of the parameter $\lambda_{c}$

$$
P_{C}\left(\lambda_{c}\right)=\frac{3-3 \lambda_{c}+3 \lambda_{c}^{2}+\lambda_{c}^{3}}{4} .
$$

\subsection{Summary of the variance value for all the cases}

In the previous section, we have establish the value of $\sigma_{\Phi_{b}}^{2}$ for all the cases. It appears that $\sigma_{\Phi_{b}}^{2}$ is always equal to a fraction of $p_{0} \frac{\phi_{0}^{2}}{6}$. The fraction is a polynomial function of a parameter $\lambda$, which represents a fraction of $\phi_{0}: \lambda \phi_{0}, \lambda \in[0,1)$. If we define an appropriate $\lambda$ for each case (see the previous section), then we can summarize the polynomial functions as follows

$$
\begin{aligned}
P_{0}\left(\lambda_{0}\right) & =\frac{\lambda_{0}^{3}}{p_{1}+p_{0} \lambda_{0}} \\
P_{A}\left(\lambda_{a}\right) & =1 \\
P_{B}\left(\lambda_{b}\right) & =1-\frac{\lambda_{b}^{3}}{4} \\
P_{C}\left(\lambda_{c}\right) & =\frac{3-3 \lambda_{c}+3 \lambda_{c}^{2}+\lambda_{c}^{3}}{4} \\
P_{D}\left(\lambda_{d}\right) & =1-\frac{\lambda_{d}^{3}}{2} \\
P_{E}\left(\lambda_{e}\right) & =\frac{1-3 \lambda_{e}+3 \lambda_{e}^{2}+\lambda_{e}^{3}}{2} .
\end{aligned}
$$

For example, for the case $\mathrm{D}, \Phi_{b}$ is expressed as the product of the theoretical bound $\left(p_{0} \frac{\phi_{0}^{2}}{6}\right)$, and $\left(1-\frac{\lambda_{d}^{3}}{2}\right)$, which is smaller than 1 , for the admissible values of its parameter. To be general, we have introduced $P_{A}\left(\lambda_{a}\right)$, which is the constant function 1 . Also, note that the values of these functions at the transition points (from one case to the next) are consistent

$$
\begin{aligned}
& P_{0}(1)=P_{A}(0)=1 \\
& P_{A}(1)=P_{B}(0)=1 \\
& P_{B}(1)=P_{C}(0)=3 / 4 \\
& P_{C}(1)=P_{D}(0)=1 \\
& P_{D}(1)=P_{E}(0)=1 / 2 \\
& P_{E}(1)=P_{A}(0)=1 .
\end{aligned}
$$


Finally, we can express the evolution of the variance w.r.t. $\phi_{W}$, and for each code (except the code 5)

$$
C_{i}, i=1,2,3,4 \quad \sigma_{\Phi_{b}}^{2}\left(\phi_{W}\right)= \begin{cases}p_{0} \frac{\phi_{0}^{2}}{6} P_{0}\left(\lambda_{0}\right) & 0 \leq \phi_{W}<\phi_{0} \\ p_{0} \frac{\phi_{0}^{2}}{6} & \phi_{0} \leq \phi_{W} \bmod \left(12 \phi_{0}\right)<i \phi_{0} \\ p_{0} \frac{\phi_{0}^{2}}{6} P_{B}\left(\lambda_{b}\right) & i \phi_{0} \leq \phi_{W} \bmod \left(12 \phi_{0}\right)<(i+1) \phi_{0} \\ p_{0} \frac{\phi_{0}^{2}}{6} P_{C}\left(\lambda_{c}\right) & (i+1) \phi_{0} \leq \phi_{W} \bmod \left(12 \phi_{0}\right)<(i+2) \phi_{0} \\ p_{0} \frac{\phi_{0}^{2}}{6} & (i+2) \phi_{0} \leq \phi_{W} \bmod \left(12 \phi_{0}\right)<(10-i) \phi_{0} \\ p_{0} \frac{\phi_{0}^{2}}{6} P_{B}\left(\lambda_{b}\right) & (10-i) \phi_{0} \leq \phi_{W} \bmod \left(12 \phi_{0}\right)<(11-i) \phi_{0} \\ p_{0} \frac{\phi_{0}^{2}}{6} P_{C}\left(\lambda_{c}\right) & (11-i) \phi_{0} \leq \phi_{W} \bmod \left(12 \phi_{0}\right)<(12-i) \phi_{0} \\ p_{0} \frac{\phi_{0}^{2}}{6} & (12-i) \phi_{0} \leq \phi_{W} \bmod \left(12 \phi_{0}\right)<11 \phi_{0} \\ p_{0} \frac{\phi_{0}^{2}}{6} P_{D}\left(\lambda_{d}\right) & 11 \phi_{0} \leq \phi_{W} \bmod \left(12 \phi_{0}\right)<12 \phi_{0} \\ p_{0} \frac{\phi_{0}^{2}}{6} P_{E}\left(\lambda_{e}\right) & 0 \leq \phi_{W} \bmod \left(12 \phi_{0}\right)<\phi_{0}, \quad \phi_{W} \geq 12 \phi_{0} .\end{cases}
$$

Note that some intervals associated to the case A may be reduced to zero (for $i=1$, and $i=4$ ) in the previous expression. Because of the particular shape of the code 5 , the period of its variance w.r.t. $\phi_{W}$ is reduced to $6 \phi_{0}$ instead of $12 \phi_{0}$. Also, it does not contain the cases B and C. Therefore, the evolution of the variance w.r.t. $\phi_{W}$ for the code 5 is expressed in a separate, but similar way

$$
C_{5}: \quad \sigma_{\Phi_{b}}^{2}\left(\phi_{W}\right)= \begin{cases}p_{0} \frac{\phi_{0}^{2}}{6} P_{0}\left(\lambda_{0}\right) & 0 \leq \phi_{W}<\phi_{0} \\ p_{0} \frac{\phi_{0}^{2}}{6} & \phi_{0} \leq \phi_{W} \bmod \left(6 \phi_{0}\right)<5 \phi_{0} \\ p_{0} \frac{\phi_{0}^{2}}{6} P_{D}\left(\lambda_{d}\right) & 5 \phi_{0} \leq \phi_{W} \bmod \left(6 \phi_{0}\right)<6 \phi_{0} \\ p_{0} \frac{\phi_{0}^{2}}{6} P_{E}\left(\lambda_{e}\right) & 0 \leq \phi_{W} \bmod \left(6 \phi_{0}\right)<\phi_{0}, \quad \phi_{W} \geq 6 \phi_{0} .\end{cases}
$$

\subsection{Simulations}

In order to validate our theory about the code statistics, we developed a simulator. The four parameters considered by the simulator are:

1. the angular window $\phi_{W}$,

2. the code (symbols and durations),

3. the turret period, and

4. the number of turret turns.

The simulations have been performed for the five codes used by BeAMS, and for values of $\phi_{W}$ ranging from 0 to $13 \phi_{0}$, in order to observe the case $0\left(\phi_{W} \in\left[0, \phi_{0}\right)\right)$, followed by a full period of the variance evolution $\left(\phi_{W} \in\left[\phi_{0}, 13 \phi_{0}\right)\right)$. The values used for the turret period and the number of turret turns are discussed in the next section.

Simulation results are presented in Figure 14 to Figure 18. From these figures, one can see that the simulations perfectly match the theory. So, in order to distinguish both curves, the theoretical variance is represented by a continuous line, and the simulated variance is represented by small unconnected circles. For convenience, we have reported the encountered case in function of the normalized angular window on the bottom of the graphs. Obviously, for all the codes, the case 0 only appears on the right, when $\phi_{W}<\phi_{0}$. For other angular window values, the relevant case is one of the possible A, B, C, D, or E cases.

In complement to the variance graphs, we have reported the pairs of observations of $\mathcal{E}_{r}$ and $\mathcal{E}_{f}$ for the different cases of the code $1^{3}$, in order to visualize the simulated supports of the

\footnotetext{
${ }^{3}$ Note that we could have use another code to observe all the different cases, except the code 5 , since it does not contain the cases $\mathrm{B}$ and $\mathrm{C}$.
} 


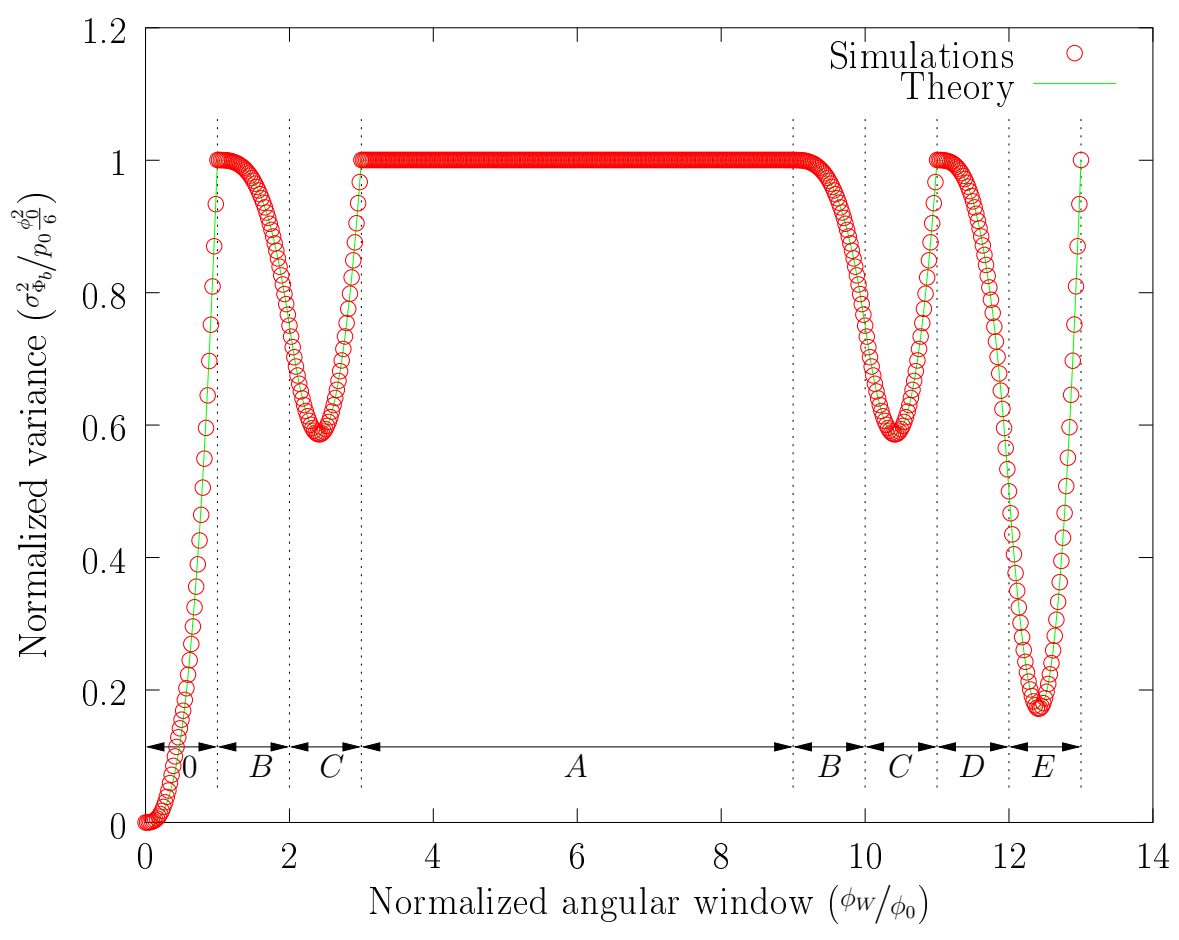

Figure 14: Variance of $\Phi_{b}$ in function of $\phi_{W}$ for the code 1: simulations versus theory.

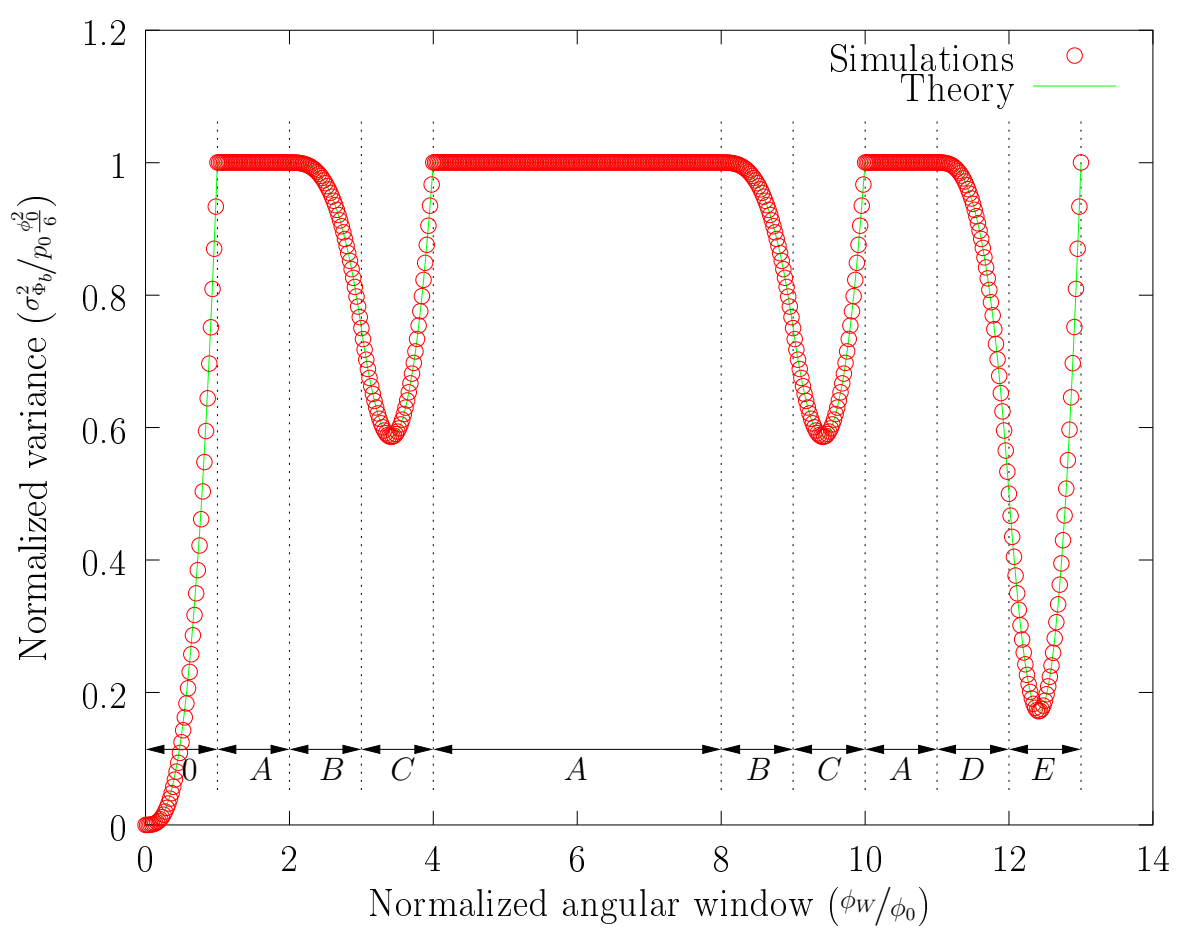

Figure 15: Variance of $\Phi_{b}$ in function of $\phi_{W}$ for the code 2: simulations versus theory. 


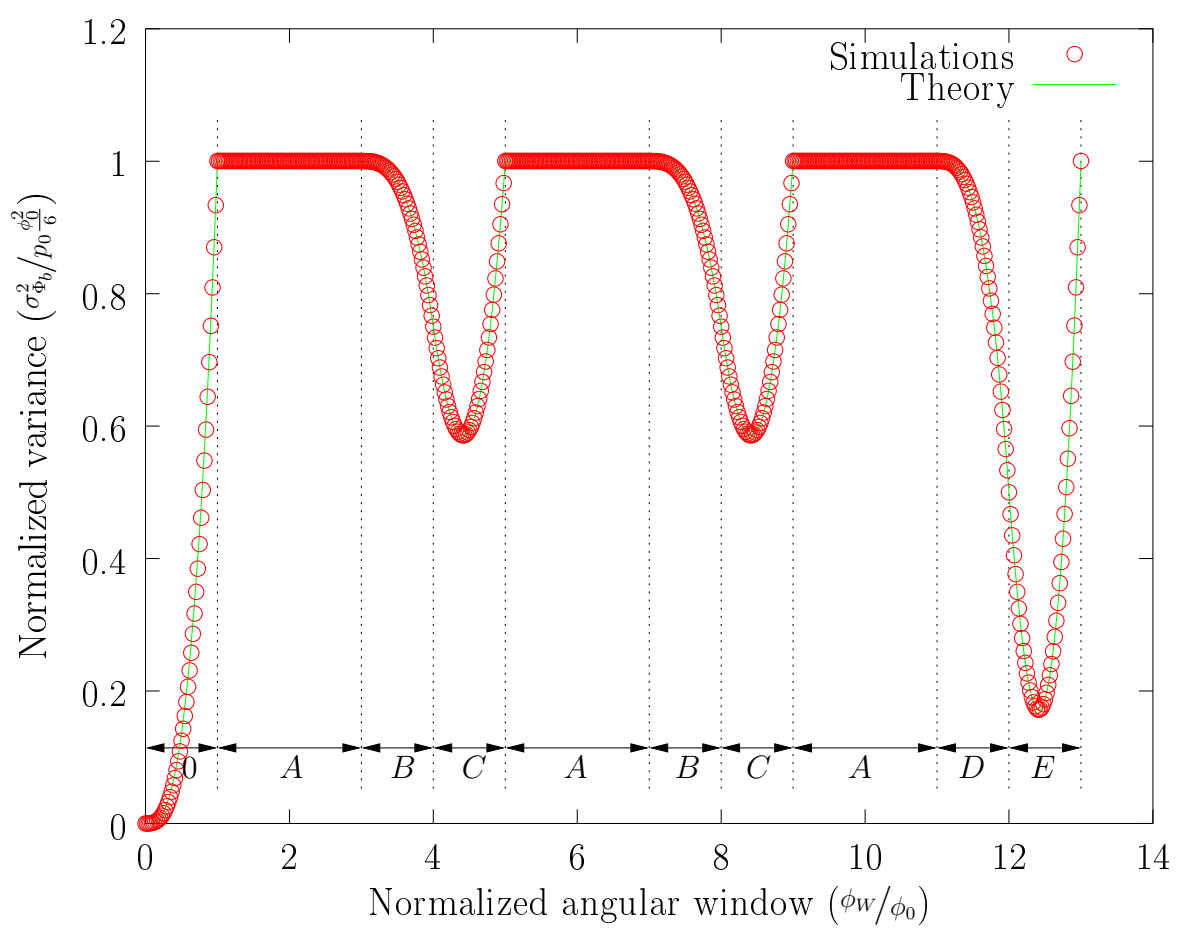

Figure 16: Variance of $\Phi_{b}$ in function of $\phi_{W}$ for the code 3: simulations versus theory.

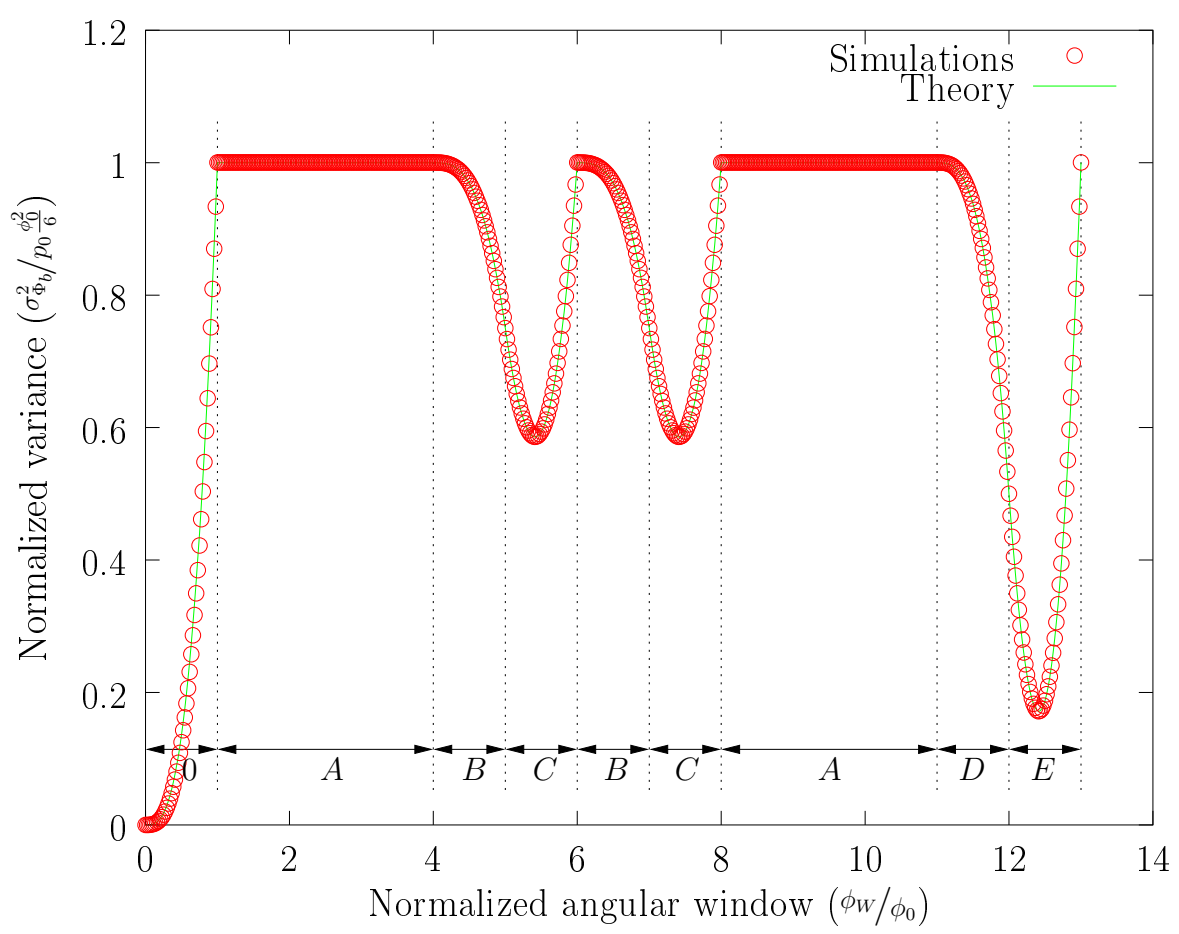

Figure 17: Variance of $\Phi_{b}$ in function of $\phi_{W}$ for the code 4: simulations versus theory. 


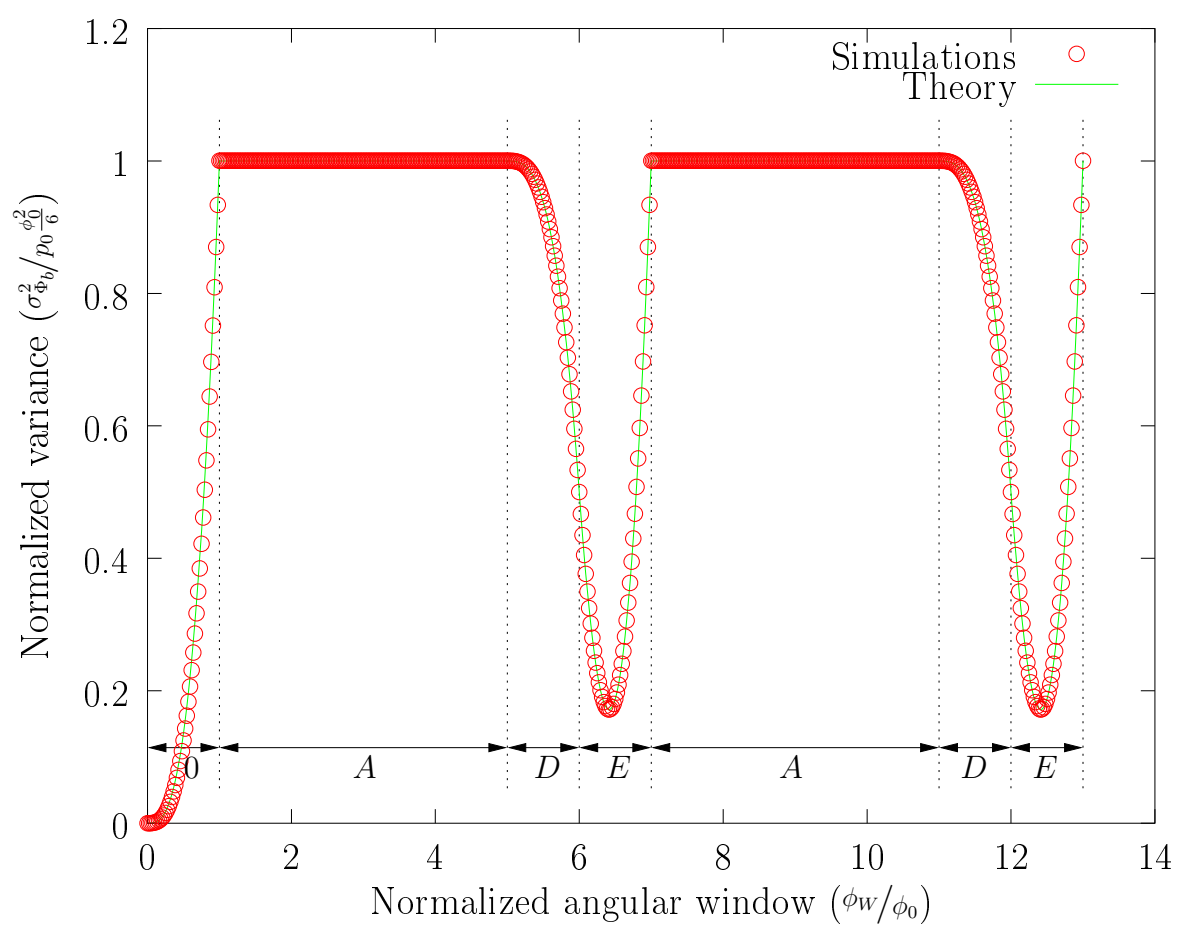

Figure 18: Variance of $\Phi_{b}$ in function of $\phi_{W}$ for the code 5: simulations versus theory.

joint $P D F s$ of $\mathcal{E}_{r}$ and $\mathcal{E}_{f}$. These supports obtained by simulation are presented in Table 4 (for all the cases, except for the case A, we have chosen values for the angular window, such that $\left.\lambda_{0}=\lambda_{b}=\lambda_{c}=\lambda_{d}=\lambda_{e}=0.5\right)$. Because the pairs of $\mathcal{E}_{r}$ and $\mathcal{E}_{f}$ values are superimposed on these graphs, it is impossible to distinguish the 2D Dirac PDF (except for the case D), and the separate parts of the cases B and C, but it appears that simulated supports also match the theory.

\subsection{Note about the time stationarity hypothesis}

To establish all the theoretical results presented in this document, we have assumed the time stationarity. In other words, these theoretical results are consistent if we can observe all the possible values for the pairs $\left(\mathcal{E}_{r}, \mathcal{E}_{f}\right)$, for all angular windows and codes. This implies that we can observe all the possible shifted versions of a code w.r.t. the angular window (like we did in the appendix to compute all the probabilities associated to the different cases).

So, in order to produce the previous figures showing the variance evolution w.r.t. the angular window, and to confirm the adequacy between theory and simulations, we had to "simulate" the time stationarity as explained hereafter. As mentioned, the simulator requires four parameters: the angular window, the code (symbols and durations), the turret period, and the number of turret turns. The two first parameters are variables of the study. However, the values of the turret period and the number of turret turns can be adjusted ${ }^{4}$ to "simulate" the time stationarity. To do so, one has to choose a value for the turret period such that the code duration and the turret period are relatively prime (or coprime). As a result, the code will shift from one unit w.r.t. the angular window, for each new observation, or turret turn. Finally, if the number of turret turns is equal to the code duration, we will observe all the possible shifted versions of the code, and the time stationarity hypothesis will be met.

\footnotetext{
${ }^{4}$ It means that we can set these parameters to values that are different from the real system.
} 
Case 0

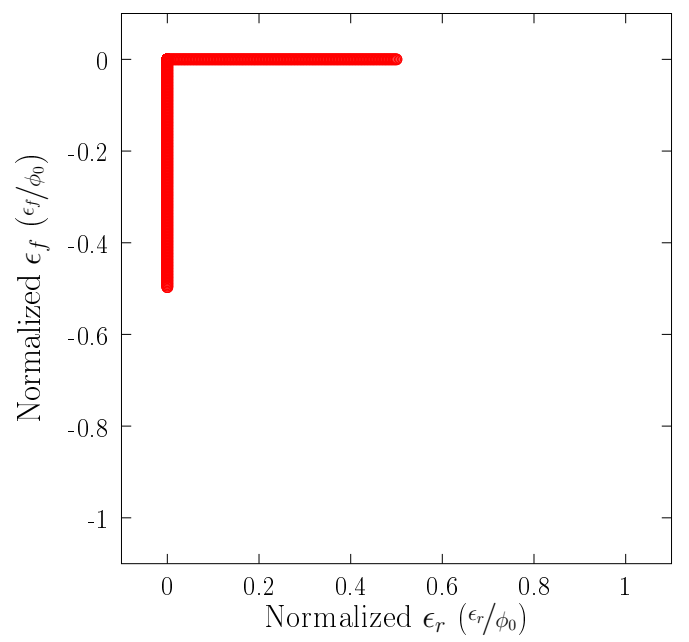

Case D

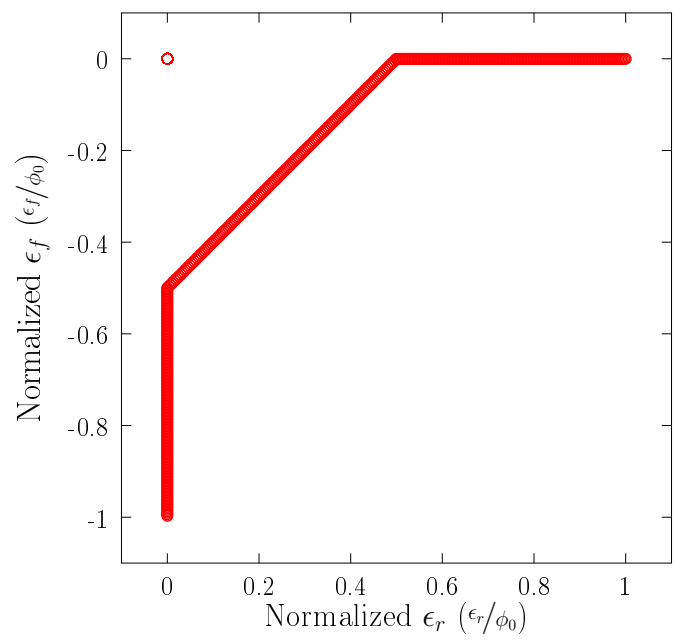

Case B

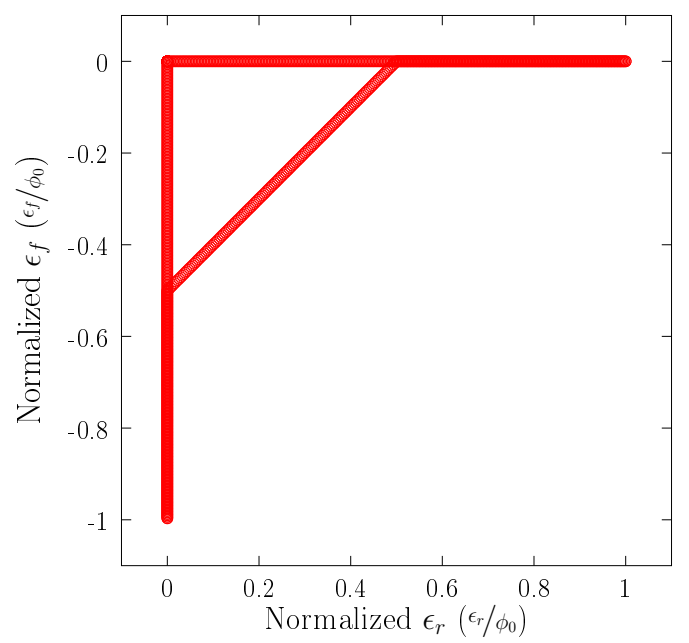

Case A

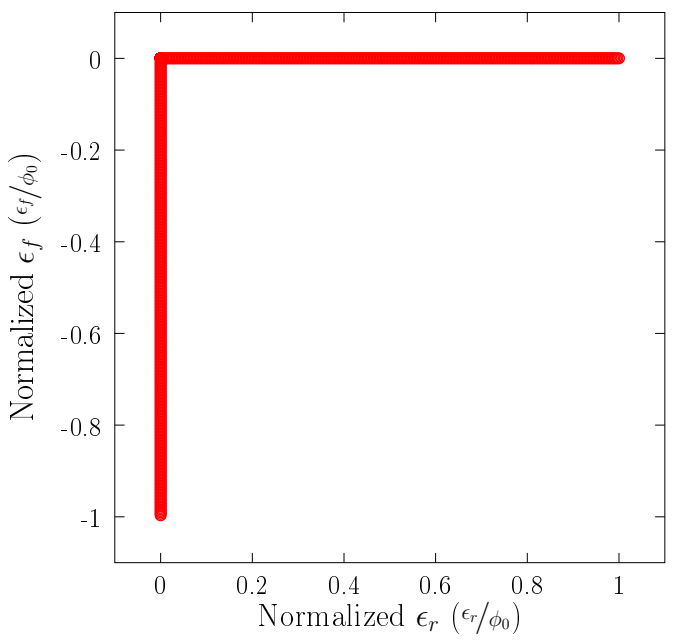

Case E

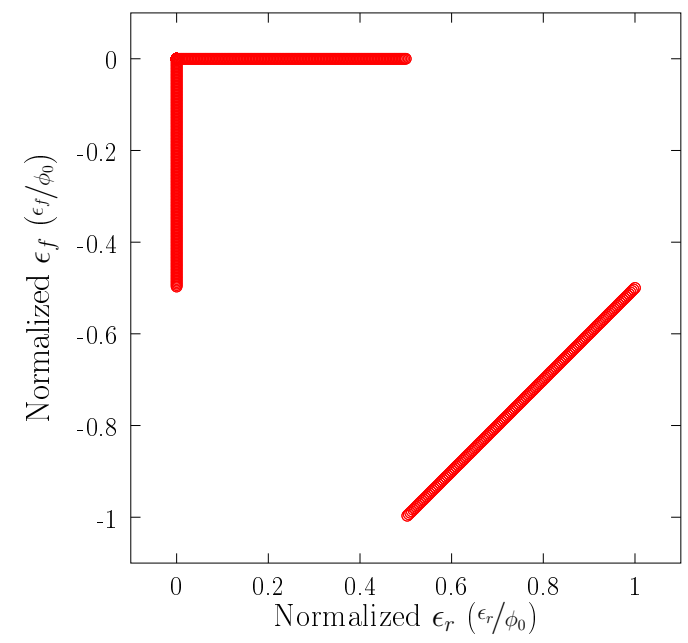

Case C

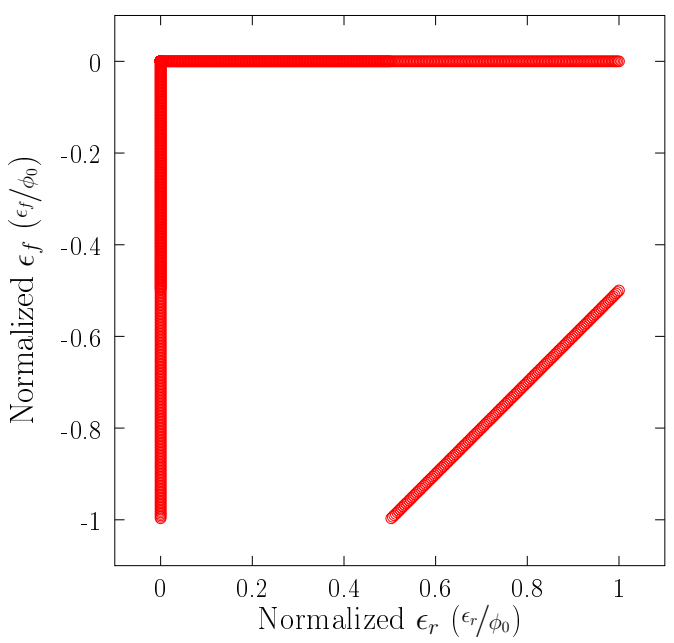

Table 4: Simulations for the supports of the joint PDFs of $\mathcal{E}_{r}$ and $\mathcal{E}_{f}$ for the 6 different Cases $\left(\lambda_{0}=\lambda_{b}=\lambda_{c}=\lambda_{d}=\lambda_{e}=0.5\right)$. 


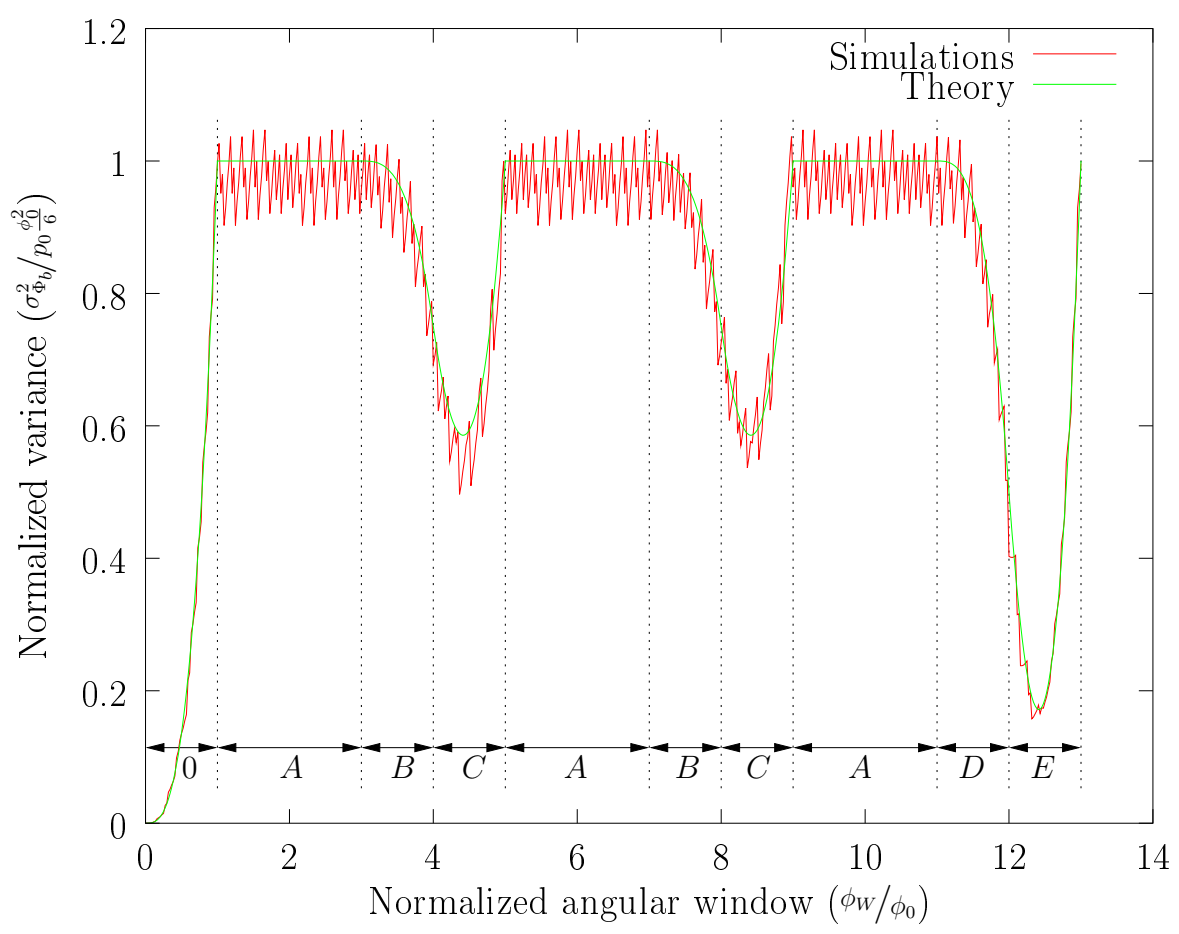

Figure 19: Variance of $\Phi_{b}$ in function of $\phi_{W}$ for the code 3: simulations versus theory (turret period $=998400$, number of turret turns $=77$ ).

In the simulator, as well as in the real system, the bit durations and the turret period are represented by integers, whose one unit is equal to $100 \mathrm{~ns}$. Therefore, the bit duration is represented by $308(30.8 \mu \mathrm{s})$, and the code duration is equal to $12 \times 308=3696$. The turret period is equal to $998400(0.09984 \mathrm{~s})$. With these values, the code duration and the turret period are not relatively prime, and this would induce some artifacts on the data. So, we changed the value of the turret period to $998401^{5}$, which is relatively prime with 3696 , in order to meet the time stationarity hypothesis, and to generate the previous graphics.

However, the turret period of BeAMS does not correspond to the one used to generate the previous graphics. The real turret period and the code duration are not relatively prime, and therefore, the time stationarity hypothesis is not met. It means that, in practice, we do not observe all the possible shifted versions of a code w.r.t. the angular window. In other terms, the simulator, and the real system, act as a "bad" pseudo random number generator. As a consequence, the pairs of observed values $\left(\mathcal{E}_{r}, \mathcal{E}_{f}\right)$ are not well balanced, and finally the computed variance of $\Phi_{b}$ can oscillate (slightly) around its theoretical value. To enlighten this phenomenon, we have performed additional simulations, with four different turret periods (998400, 999000, 999200, and 999300 respectively), all not relatively prime with the code duration. The results are presented in Figure 19 to Figure 22. From these figures, one can observe the oscillations of the variance of $\Phi_{b}$. As a consequence, the variance may exceed the theoretical bound established in this document. Unfortunately, it is difficult to formalize how the variance can exceed the theoretical bound, since it depends on the code symbols, the code duration, and the turret period. However the simulator can always help in finding all these characteristics. In the case of BeAMS, this variance can exceed the theoretical bound by up to $14 \%$ for the code 2 and 4 . Finally, note that the number of turret turns has been chosen such that we cover a complete period of observations of the code through

\footnotetext{
${ }^{5}$ Note that we chose the closest relatively prime integer, but we could have chosen any other relatively prime integer.
} 


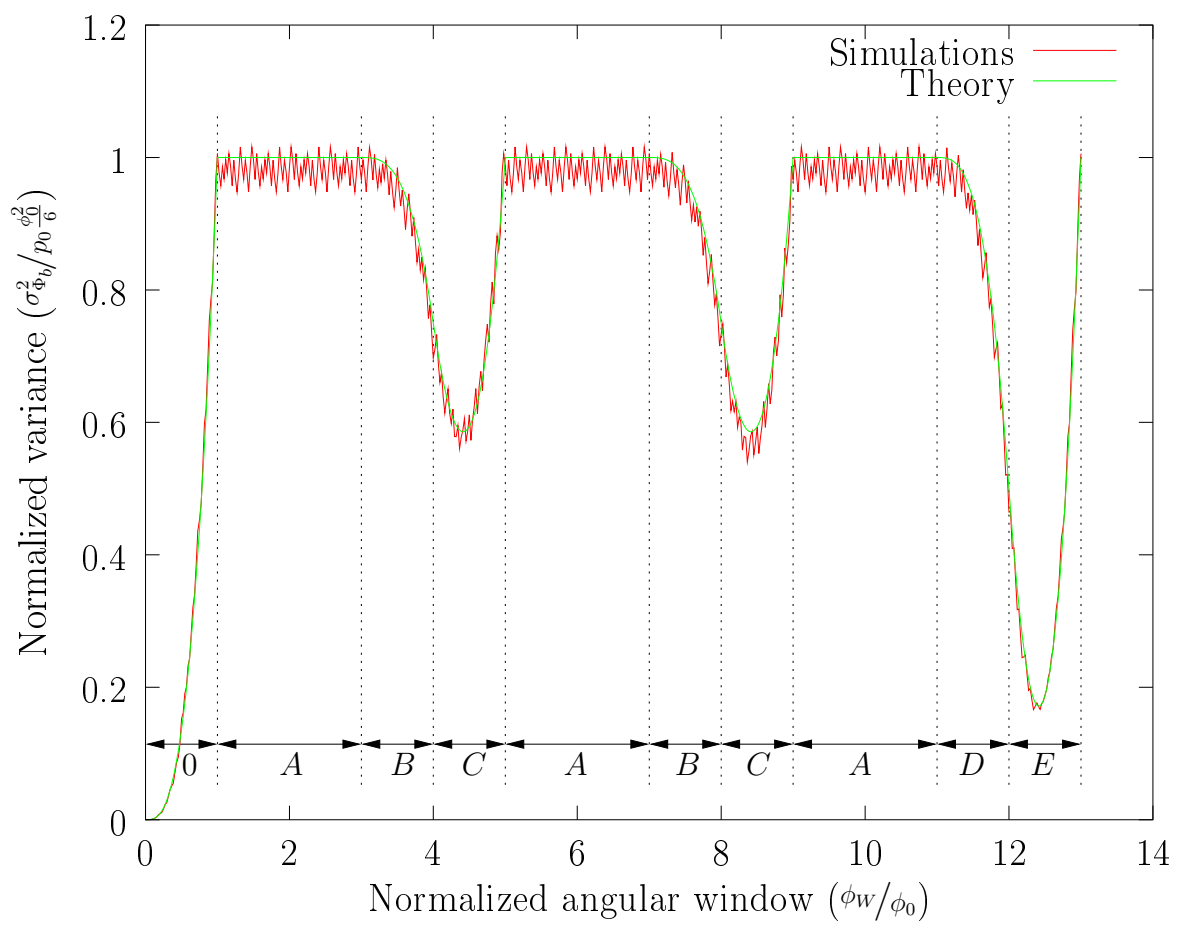

Figure 20: Variance of $\Phi_{b}$ in function of $\phi_{W}$ for the code 3: simulations versus theory (turret period $=999000$, number of turret turns $=154$ ).

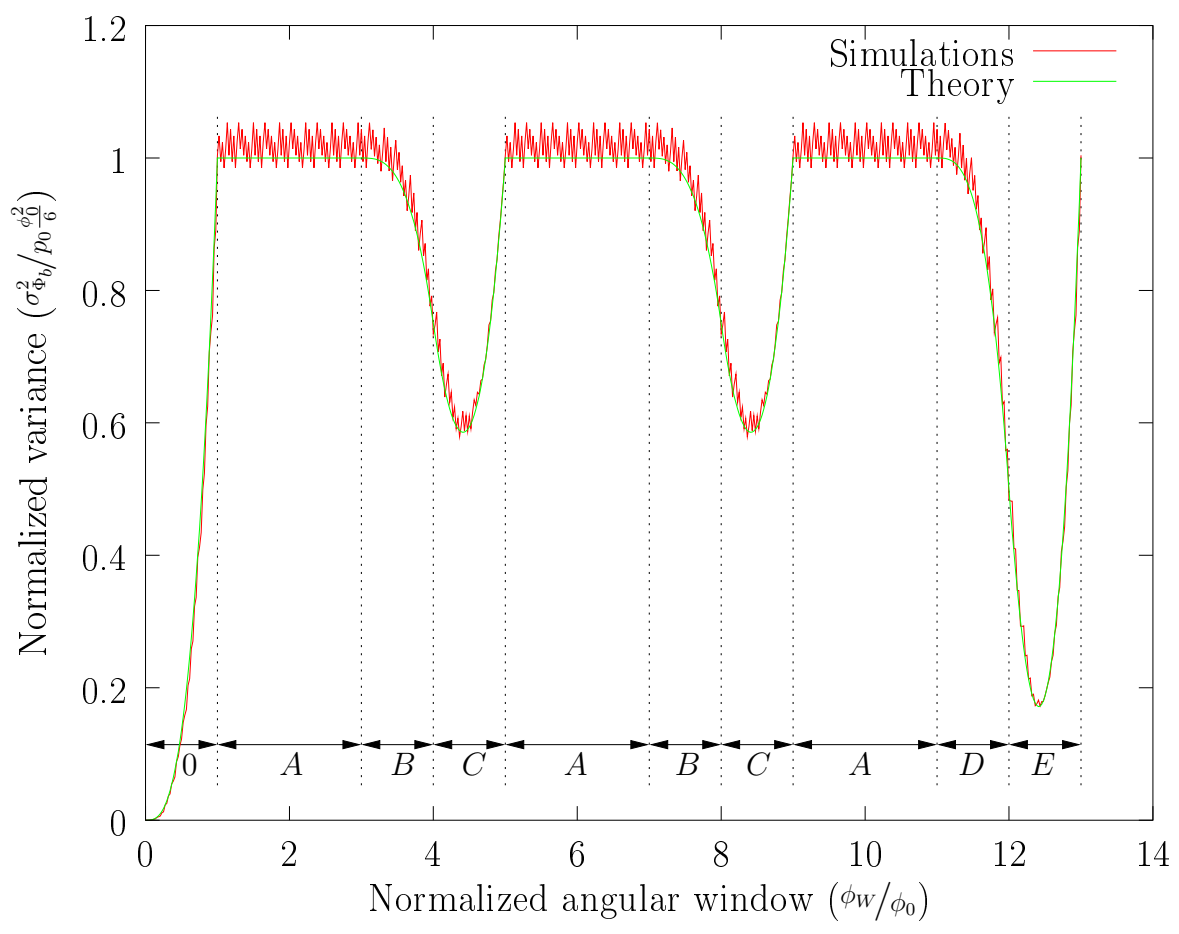

Figure 21: Variance of $\Phi_{b}$ in function of $\phi_{W}$ for the code 3: simulations versus theory (turret period $=999200$, number of turret turns $=231$ ). 


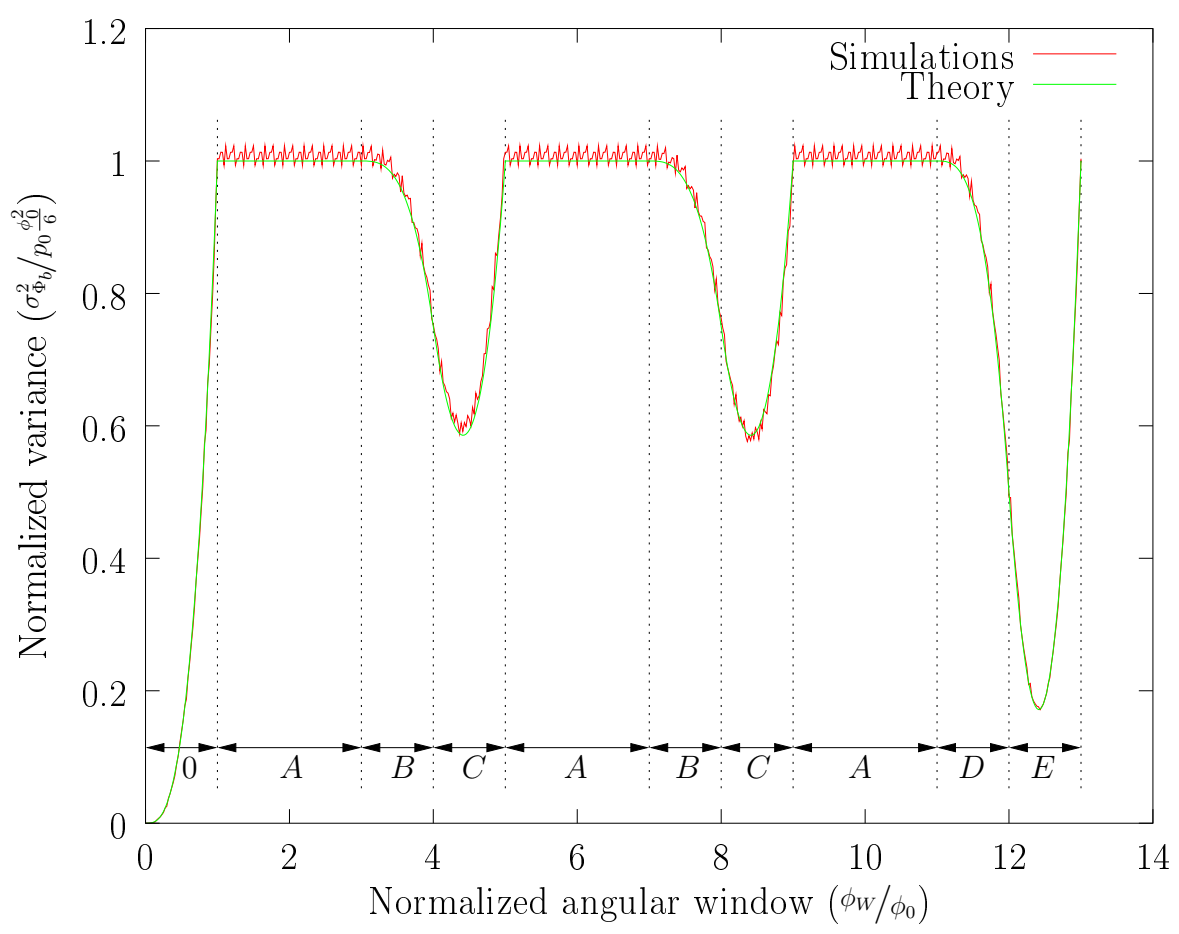

Figure 22: Variance of $\Phi_{b}$ in function of $\phi_{W}$ for the code 3: simulations versus theory (turret period $=999300$, number of turret turns $=308)$.

the angular window

$$
\text { number of turret turns }=\frac{\text { codeduration }}{\operatorname{gcd}(\text { codeduration, } \text { turret period })},
$$

where $\operatorname{gcd}(a, b)$ is the greatest common divisor of $a$ and $b$. Also, one can observe from these figures, that the oscillations in the simulated variances decrease if the number of turret turns require to cover a complete period of observations increases. The theoretical time stationarity condition is reached when $\operatorname{gcd}($ codeduration, turret period) is equal to 1 , that is, when the code duration and the turret period are relatively prime.

\section{Conclusions}

In [4], we have proposed a new angle measurement system, named BeAMS, that is the key element of an algorithm for mobile robot positioning. BeAMS introduces a new mechanism to measure angles: it detects a beacon when it enters and leaves an angular window. One of the major challenges of such a system is to be able to model its behavior from a statistical point of view in order to both have an adequate measure of its efficiency and appropriate values to feed a tracking system (a Kalman filter for instance).

A theoretical framework for a thorough performance analysis of BeAMS has been provided in [4] to establish the upper bound of the variance, and to validate this bound through experiments and simulations. It has been shown that the estimator derived from the center of this angular window provides an unbiased estimate of the beacon angle.

This document complements our paper by going into further details related to the code statistics of modulated signals in general, with an emphasis on BeAMS. In particular, the probability density function of the measured angle has been previously established with the assumption that there is no 
correlation between the times a beacon enters the angular window or leaves it. This assumption is questionable and, in this document, we reconsider this assumption and establish the exact probability density function of the angle estimated by BeAMS (without this assumption).

The conclusion of this study is that the real variance of the estimator provided by BeAMS was slightly underestimated in our previous work. In addition to this specific result, we also provide a new and extensive theoretical approach that can be used to analyze the statistics of any angle measurement method with beacons whose signal has been modulated.

In this document, we have developed four major ideas:

1. it is possible to establish the exact probability density function of the angle estimator of BeAMS.

2. a practical upper bound of the variance of this estimator has been established, which is of practical interest for calibration and tracking (see Table 1, on page 13, for a summary),

3. there are many particular cases for analyzing BeAMS or, more generally, any angle measurement based on the emission of modulated (coded) signals. Therefore, we have to develop a new theoretical approach to evaluate the performance of systems, and to clarify all possible cases.

4. it is shown that the variance evolves as a function of the angular window (while remaining below the upper bound). This is essential for any system that measures angles. Often, authors consider that the variance evolves with the distance. However, our analysis is preferable, because the angular window is measurable by the system even if the robot position is unknown. Our analysis has a direct usage for any practical situation.

Because the developments given in this document are very complete, it is possible to really understand the behavior of the system and to improve the design of BeAMS. Further extensions concern improved calibration procedures, better estimate, and an enhanced tracking of the robot position over time.

To conclude this document, we believe it is important to mention that, although this study was carried on to understand and improve BeAMS, the theoretical framework is larger than that of BeAMS. It is applicable to any estimator that is built like that of BeAMS. In particular, it is applicable to any measurement system that estimates a value by taking the mean of a previous event and a later event, based on the reception of an On-Off Keying modulated signal. For example, the study of the different "Cases" is general and extensible to any code. Likewise, if measures on the events are symmetric, then the mean is always unbiased. 


\section{A Some details}

\section{A.1 Mean and variance of a random variable whose $P D F$ is expressed as a weighted sum (mixture) of PDFs}

Let $X$ be a random variable with a probability density function $(P D F) f_{X}(x)$

$$
X \sim f_{X}(x) .
$$

Suppose that $f_{X}(x)$ can be written as a weighted sum (mixture) of functions $f_{i}(x)$

$$
f_{X}(x)=\sum_{i} k_{i} f_{i}(x),
$$

whose coefficients $k_{i}$ sum up to 1

$$
\sum_{i} k_{i}=1
$$

and that the area of each $f_{i}(x)$ is equal to 1

$$
\int_{-\infty}^{+\infty} f_{i}(x) d x=1
$$

so that we have

$$
\int_{-\infty}^{+\infty} f_{X}(x) d x=\int_{-\infty}^{+\infty} \sum_{i} k_{i} f_{X_{i}}(x) d x=\sum_{i} k_{i} \int_{-\infty}^{+\infty} f_{X_{i}}(x) d x=1,
$$

The existence theorem (see [1, page 73]) states the following.

Theorem 2 If $f_{i}(x)$ is nonnegative, if its area is equal to 1 , if its integral $F_{i}(x)$ is continuous from the right, and if, as $x$ increases from $-\infty$ to $\infty, F_{i}(x)$ increases monotonically from 0 to 1 , then we can define a random variable $X_{i}$ whose $\mathrm{PDF}$ is $f_{i}(x)$

$$
X_{i} \sim f_{i}(x)=f_{X_{i}}(x) .
$$

Then the expectation of $X$ is given by

$$
E\{X\}=\sum_{i} k_{i} E\left\{X_{i}\right\}
$$

and the variance of $X$ is given either by

$$
\operatorname{var}\{X\}=\sum_{i} k_{i}\left[\operatorname{var}\left\{X_{i}\right\}+E\left\{X_{i}\right\}^{2}\right]-(E\{X\})^{2},
$$

or by

$$
\operatorname{var}\{X\}=\sum_{i} k_{i} \operatorname{var}\left\{X_{i}\right\}+\sum_{i<j} k_{i} k_{j}\left(E\left\{X_{i}\right\}-E\left\{X_{j}\right\}\right)^{2}
$$

Proof For the mean, we have

$$
\begin{aligned}
E\{X\} & =\int_{-\infty}^{+\infty} x f_{X}(x) d x \\
& =\int_{-\infty}^{+\infty} x \sum_{i} k_{i} f_{X_{i}}(x) d x \\
& =\sum_{i} k_{i} \int_{-\infty}^{+\infty} x f_{X_{i}}(x) d x \\
& =\sum_{i} k_{i} E\left\{X_{i}\right\}
\end{aligned}
$$


The first expression of the variance is obtained as follows

$$
\begin{aligned}
\operatorname{var}\{X\} & =\int_{-\infty}^{+\infty}(x-E\{X\})^{2} f_{X}(x) d x \\
& =\int_{-\infty}^{+\infty} x^{2} f_{X}(x) d x-(E\{X\})^{2} \\
& =\int_{-\infty}^{+\infty} x^{2} \sum_{i} k_{i} f_{X_{i}}(x) d x-(E\{X\})^{2} \\
& =\sum_{i} k_{i} \int_{-\infty}^{+\infty} x^{2} f_{X_{i}}(x) d x-(E\{X\})^{2} \\
& =\sum_{i} k_{i}\left[\operatorname{var}\left\{X_{i}\right\}+E\left\{X_{i}\right\}^{2}\right]-(E\{X\})^{2},
\end{aligned}
$$

and the second expression of the variance is obtained by starting from the previous result

$$
\begin{aligned}
& \operatorname{var}\{X\}=\sum_{i} k_{i}\left[\operatorname{var}\left\{X_{i}\right\}+E\left\{X_{i}\right\}^{2}\right]-(E\{X\})^{2} \\
& =\sum_{i} k_{i}\left[\operatorname{var}\left\{X_{i}\right\}+E\left\{X_{i}\right\}^{2}\right]-\left(\sum_{i} k_{i} E\left\{X_{i}\right\}\right)^{2} \\
& =\sum_{i} k_{i}\left[\operatorname{var}\left\{X_{i}\right\}+E\left\{X_{i}\right\}^{2}\right]-\left[\sum_{i} k_{i}^{2} E\left\{X_{i}\right\}^{2}+\sum_{i<j} 2 k_{i} k_{j} E\left\{X_{i}\right\} E\left\{X_{j}\right\}\right] \\
& =\sum_{i} k_{i} \operatorname{var}\left\{X_{i}\right\}+\left[\sum_{i} k_{i} E\left\{X_{i}\right\}^{2}-\sum_{i} k_{i}^{2} E\left\{X_{i}\right\}^{2}\right]-\sum_{i<j} 2 k_{i} k_{j} E\left\{X_{i}\right\} E\left\{X_{j}\right\} \\
& =\sum_{i} k_{i} \operatorname{var}\left\{X_{i}\right\}+\sum_{i} k_{i}\left(1-k_{i}\right) E\left\{X_{i}\right\}^{2}-\sum_{i<j} 2 k_{i} k_{j} E\left\{X_{i}\right\} E\left\{X_{j}\right\} \\
& =\sum_{i} k_{i} \operatorname{var}\left\{X_{i}\right\}+\sum_{i} k_{i} \sum_{j, j \neq i} k_{j} E\left\{X_{i}\right\}^{2}-\sum_{i<j} 2 k_{i} k_{j} E\left\{X_{i}\right\} E\left\{X_{j}\right\} \\
& =\sum_{i} k_{i} \operatorname{var}\left\{X_{i}\right\}+\sum_{j \neq i} k_{i} k_{j} E\left\{X_{i}\right\}^{2}-\sum_{i<j} 2 k_{i} k_{j} E\left\{X_{i}\right\} E\left\{X_{j}\right\} \\
& =\sum_{i} k_{i} \operatorname{var}\left\{X_{i}\right\}+\sum_{i<j} k_{i} k_{j} E\left\{X_{i}\right\}^{2}+\sum_{j<i} k_{i} k_{j} E\left\{X_{i}\right\}^{2}-\sum_{i<j} 2 k_{i} k_{j} E\left\{X_{i}\right\} E\left\{X_{j}\right\} \\
& =\sum_{i} k_{i} \operatorname{var}\left\{X_{i}\right\}+\sum_{i<j} k_{i} k_{j} E\left\{X_{i}\right\}^{2}+\sum_{i<j} k_{j} k_{i} E\left\{X_{j}\right\}^{2}-\sum_{i<j} 2 k_{i} k_{j} E\left\{X_{i}\right\} E\left\{X_{j}\right\} \\
& =\sum_{i} k_{i} \operatorname{var}\left\{X_{i}\right\}+\sum_{i<j} k_{i} k_{j}\left(E\left\{X_{i}\right\}^{2}+E\left\{X_{j}\right\}^{2}-2 E\left\{X_{i}\right\} E\left\{X_{j}\right\}\right) \\
& =\sum_{i} k_{i} \operatorname{var}\left\{X_{i}\right\}+\sum_{i<j} k_{i} k_{j}\left(E\left\{X_{i}\right\}-E\left\{X_{j}\right\}\right)^{2}
\end{aligned}
$$

\section{A.2 Means and variances of $\Phi_{r}$ and $\Phi_{f}$}

\section{A.2.1 Mean and variance of $\Phi_{r}$}

The $P D F$ of $\Phi_{r}$ is given by

$$
f_{\Phi_{r}}(\phi)=p_{1} \delta\left(\phi-\phi_{R}\right)+p_{0} U_{\left(\phi_{R}, \phi_{R}+\phi_{0}\right)}(\phi) .
$$


By using (123), we can compute the mean of $\Phi_{r}$

$$
\begin{aligned}
\mu_{\Phi_{r}} & =p_{1} \phi_{R}+p_{0}\left(\phi_{R}+\frac{\phi_{0}}{2}\right) \\
& =\phi_{R}+p_{0} \frac{\phi_{0}}{2} .
\end{aligned}
$$

The variance of $\Phi_{r}$ is computed after (125)

$$
\begin{aligned}
\sigma_{\Phi_{r}}^{2} & =p_{1} 0+p_{0} \frac{\phi_{0}^{2}}{12}+p_{1} p_{0}\left(\phi_{R}-\left(\phi_{R}+\frac{\phi_{0}}{2}\right)\right)^{2} \\
& =p_{0} \frac{\phi_{0}^{2}}{12}+\left(1-p_{0}\right) p_{0}\left(\frac{\phi_{0}}{2}\right)^{2} \\
& =p_{0} \frac{\phi_{0}^{2}}{12}+p_{0} \frac{\phi_{0}^{2}}{4}-p_{0}^{2} \frac{\phi_{0}^{2}}{4} \\
& =p_{0} \frac{\phi_{0}^{2}}{3}-p_{0}^{2} \frac{\phi_{0}^{2}}{4}
\end{aligned}
$$

\section{A.2.2 Mean and variance of $\Phi_{f}$}

The $P D F$ of $\Phi_{f}$ is given by

$$
f_{\Phi_{f}}(\phi)=p_{1} \delta\left(\phi-\phi_{F}\right)+p_{0} U_{\left(\phi_{F}-\phi_{0}, \phi_{F}\right)}(\phi) .
$$

By using (123), we can compute the mean of $\Phi_{f}$

$$
\begin{aligned}
\mu_{\Phi_{f}} & =p_{1} \phi_{F}+p_{0}\left(\phi_{F}-\frac{\phi_{0}}{2}\right) \\
& =\phi_{F}-p_{0} \frac{\phi_{0}}{2} .
\end{aligned}
$$

The variance of $\Phi_{f}$ is computed after (125)

$$
\begin{aligned}
\sigma_{\Phi_{f}}^{2} & =p_{1} 0+p_{0} \frac{\phi_{0}^{2}}{12}+p_{1} p_{0}\left(\phi_{F}-\left(\phi_{F}-\frac{\phi_{0}}{2}\right)\right)^{2} \\
& =p_{0} \frac{\phi_{0}^{2}}{12}+\left(1-p_{0}\right) p_{0}\left(\frac{\phi_{0}}{2}\right)^{2} \\
& =p_{0} \frac{\phi_{0}^{2}}{12}+p_{0} \frac{\phi_{0}^{2}}{4}-p_{0}^{2} \frac{\phi_{0}^{2}}{4} \\
& =p_{0} \frac{\phi_{0}^{2}}{3}-p_{0}^{2} \frac{\phi_{0}^{2}}{4}
\end{aligned}
$$

\section{A.3 Details for the case A}

The purpose of this section is to establish the $p^{\bullet}$ and $p^{\star}$ probabilities.

To compute these probabilities, we have to measure the total duration for which the different events occur over a code period, and then divide by the code period (we can do this since the codes are periodic). The situation is depicted in Figure 23. The scheme represents the generalized case, for any code $i$. We have to shift the code from left to right (for a fixed value of the angular window), and check when the different events occur, until we have analyzed the complete period of the code. We can distinguish between 8 different scenarios $(1 \rightarrow 8)$ depending on the angular shift: 


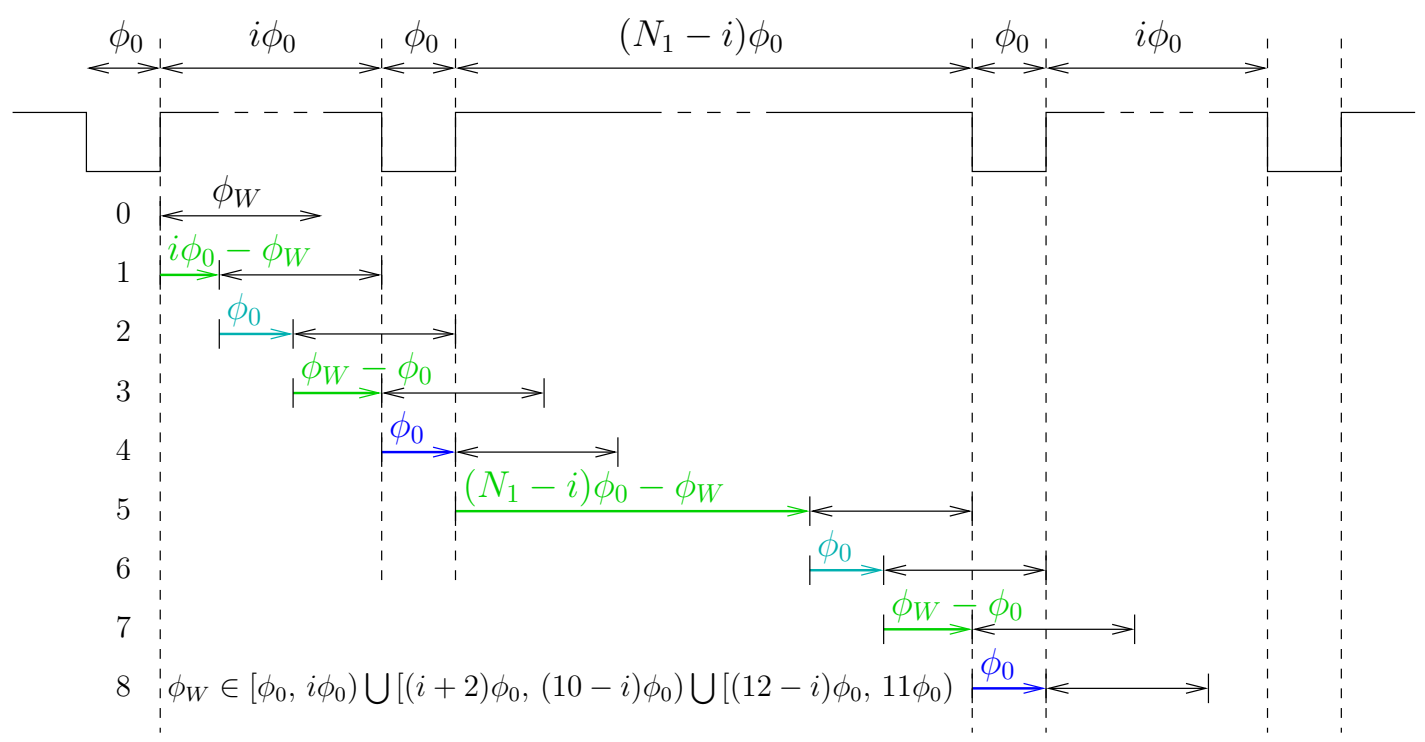

Figure 23: Computation of the probabilities and intervals associated with the case A, and for all codes ( $\phi_{W}$ is supposed to be constant).

- 0: initial condition: the left extremity of $\phi_{W}$ coincides with the start of a code period (no shift),

- 1: we can shift $\phi_{W}$ for $i \phi_{0}-\phi_{W}$ with no error,

- 2: for the next $\phi_{0}$ shift, there is an error on $\Phi_{f}$,

- 3: we can shift $\phi_{W}$ for $\phi_{W}-\phi_{0}$ with no error,

- 4: for the next $\phi_{0}$ shift, there is an error on $\Phi_{r}$,

- 5: we can shift $\phi_{W}$ for $\left(N_{1}-i\right) \phi_{0}-\phi_{W}$ with no error,

- 6: for the next $\phi_{0}$ shift, there is an error on $\Phi_{f}$,

- 7: we can shift $\phi_{W}$ for $\phi_{W}-\phi_{0}$ with no error,

- 8: for the next $\phi_{0}$ shift, there is an error on $\Phi_{r}$.

Note that in Figure 23, as for all other cases (Figures 24, 25, 26, 27, 28), we use the following color convention:

- green: no error,

- cyan: $\mathcal{E}_{r}=0$ and $\mathcal{E}_{f} \neq 0$,

- blue: $\mathcal{E}_{r} \neq 0$ and $\mathcal{E}_{f}=0$,

- red: $\mathcal{E}_{r} \neq 0$ and $\mathcal{E}_{f} \neq 0$ (for the cases B, C, D, and E only),

- brown: no edge detection at the receiver (for the case 0 only).

Now we add the favorable parts (lines $1,3,5$, and 7 , or green parts of the drawing) to find the duration of the event associated to $p^{\bullet}$

$$
\phi^{\bullet}=\left(i \phi_{0}-\phi_{W}\right)+\left(\phi_{W}-\phi_{0}\right)+\left(\left(N_{1}-i\right) \phi_{0}-\phi_{W}\right)+\left(\phi_{W}-\phi_{0}\right)=N_{1} \phi_{0}-N_{0} \phi_{0} .
$$




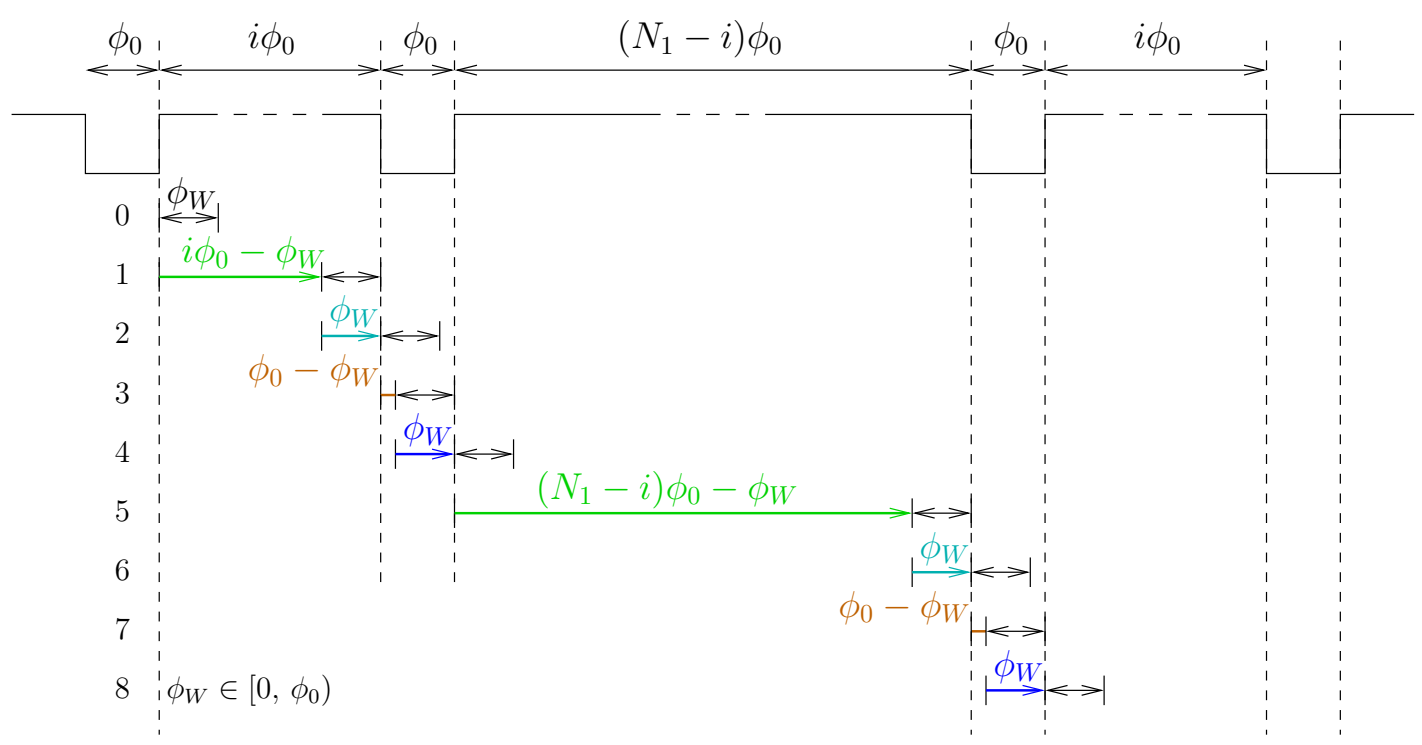

Figure 24: Computation of the probabilities and intervals associated with the case 0 , and for all codes $\left(\lambda_{0}=0.8\right.$ in the scheme).

To compute $p^{\bullet}$, we have to divide this duration by the period duration of a code $N_{b} \phi_{0}=\left(N_{1}+\right.$ $\left.N_{0}\right) \phi_{0}$

$$
p^{\bullet}=\frac{N_{1} \phi_{0}-N_{0} \phi_{0}}{N_{b} \phi_{0}}=\frac{N_{1}}{N_{b}}-\frac{N_{0}}{N_{b}}=p_{1}-p_{0} .
$$

Firstly, we can notice that this probability does not depend on the angular window $\phi_{W}$ (if the value belongs to the intervals defined for the case A). Secondly, we notice that this probability does not depend on the code. Of course the case A for the other codes does not occur for the same intervals, but the probabilities, and the PDFs are the same.

Now we have to compute $p^{\star}$. Let us consider the errors on $\Phi_{f}$ first. We can use the same reasoning as before and use Figure 23 to count favorable and unfavorable situations. In this case, the favorable parts are the cyan parts of lines 2 and 6 . The counting gives

$$
\phi^{\star}=2 \phi_{0}=N_{0} \phi_{0},
$$

and the probability $p^{\star}$ to commit an error on $\Phi_{f}$ is obtained by dividing it by the period duration

$$
p^{\star}=\frac{N_{0} \phi_{0}}{N_{b} \phi_{0}}=\frac{N_{0}}{N_{b}}=p_{0} .
$$

The same reasoning also applies to $\Phi_{r}$, but for the blue parts of line 4 and 8 . The probability to commit an error on $\Phi_{r}$ is the same as for $\Phi_{f}$, explaining why the notation $p^{\star}$ is the same for both. We can check that the probabilities for the case A sum up to 1

$$
p^{\bullet}+p^{\star}+p^{\star}=p_{1}-p_{0}+p_{0}+p_{0}=p_{1}+p_{0}=1 .
$$

\section{A.4 Details for the case 0}

The probabilities $p^{\bullet}$ and $p^{*}$ are computed as follows. To compute these probabilities, we have to measure the total duration for which the different events occur over a code period, and then divide by the code period (we can do this since the codes are periodic). The situation is depicted in Figure 24. 
The scheme represents the generalized case, for any code $i$. One has to move virtually the code from left to right (for a fixed value of the angular window) and check when the different events occur, until we reach the period of the code. We can distinguish between 8 different scenarios depending on the angular shift:

- 0: initial condition: the left extremity of $\phi_{W}$ coincides with the start of a code period (no shift),

- 1: we can shift $\phi_{W}$ for $i \phi_{0}-\phi_{W}$ with no error,

- 2: then we can shift $\phi_{W}$ for a duration $\phi_{W}$, with an error on $\Phi_{f}$ only,

- 3: for the next $\phi_{0}-\phi_{W}$ move, there is no edge detection at the receiver since there is no 1 symbol between the rising and falling edge,

- 4: then we can shift $\phi_{W}$ for a duration $\phi_{W}$, with an error on $\Phi_{r}$ only,

- 5: we can shift $\phi_{W}$ for $\left(N_{1}-i\right) \phi_{0}-\phi_{W}$ with no error,

- 6: same as line 2,

- 7: same as line 3,

- 8: same as line 4 .

This is very similar to the case A, except that there are two parts (lines 3 and 7 , or brown parts of the drawing) where there is no edge detection at all. To compute $p^{\bullet}$, we first add the favorable parts (lines 1 , and 5 , or green parts of the drawing)

$$
\phi^{\bullet}=\left(i \phi_{0}-\phi_{W}\right)+\left(\left(N_{1}-i\right) \phi_{0}-\phi_{W}\right)=N_{1} \phi_{0}-2 \phi_{W}=N_{1} \phi_{0}-N_{0} \phi_{W}=N_{1} \phi_{0}-N_{0} \phi_{0} \lambda_{0} .
$$

But unlike the case A (and all other cases), we cannot divide this duration by the code duration. Indeed, we have to remove from the code duration the parts where there is no edge detection (lines 3 and 7 , or brown parts of the drawing)

$$
\begin{aligned}
\phi_{\text {duration-case } 0} & =\left(N_{1}+N_{0}\right) \phi_{0}-2\left(\phi_{0}-\phi_{W}\right) \\
& =\left(N_{1}+N_{0}\right) \phi_{0}-N_{0}\left(\phi_{0}-\phi_{W}\right) \\
& =\left(N_{1}+N_{0}\right) \phi_{0}-N_{0} \phi_{0}\left(1-\lambda_{0}\right) \\
& =N_{1} \phi_{0}+N_{0} \phi_{0} \lambda_{0} .
\end{aligned}
$$

Now we can compute $p^{\bullet}$

$$
p^{\bullet}=\frac{\phi^{\bullet}}{\phi_{\text {duration-case } 0}}=\frac{N_{1} \phi_{0}-N_{0} \phi_{0} \lambda_{0}}{N_{1} \phi_{0}+N_{0} \phi_{0} \lambda_{0}}=\frac{p_{1}-p_{0} \lambda_{0}}{p_{1}+p_{0} \lambda_{0}} .
$$

Now, it remains to calculate $p^{*}$. Let us consider the errors on $\Phi_{f}$ first. We can use the same reasoning as before and use Figure 24 to do the counting. But in this case, the favorable parts are the cyan parts of lines 2 and 6 . The counting gives

$$
\phi^{*}=2 \phi_{W}=N_{0} \phi_{W}=N_{0} \phi_{0} \lambda_{0},
$$

and the probability $p^{*}$ to commit an error on $\Phi_{f}$ is obtained by dividing $\phi^{*}$ by the reduced period duration

$$
p^{*}=\frac{\phi^{*}}{\phi_{\text {duration-case } 0}}=\frac{N_{0} \phi_{0} \lambda_{0}}{N_{1} \phi_{0}+N_{0} \phi_{0} \lambda_{0}}=\frac{p_{0} \lambda_{0}}{p_{1}+p_{0} \lambda_{0}} .
$$

The same reasoning also applies to $\Phi_{r}$, but for the blue parts of line 4 and 8 . The probability to commit an error on $\Phi_{r}$ is the same as for $\Phi_{f}$, explaining why the notation $p^{*}$ is the same for both. Again, we can check that the probabilities for the case 0 sum up to 1

$$
p^{\bullet}+p^{*}+p^{*}=\frac{p_{1}-p_{0} \lambda_{0}}{p_{1}+p_{0} \lambda_{0}}+2 \frac{p_{0} \lambda_{0}}{p_{1}+p_{0} \lambda_{0}}=\frac{p_{1}+p_{0} \lambda_{0}}{p_{1}+p_{0} \lambda_{0}}=1 .
$$


The computation of the joint expectation is detailed hereafter

$$
\begin{aligned}
E\left\{\mathcal{E}_{r}, \mathcal{E}_{f}\right\}= & \iint_{-\infty}^{+\infty} \epsilon_{r} \epsilon_{f} f_{\mathcal{E}_{r} \mathcal{E}_{f}}\left(\epsilon_{r}, \epsilon_{f}\right) d \epsilon_{r} d \epsilon_{f} \\
= & \frac{p_{1}-p_{0} \lambda_{0}}{p_{1}+p_{0} \lambda_{0}} \iint_{-\infty}^{+\infty} \epsilon_{r} \epsilon_{f} \delta\left(\epsilon_{r}\right) \delta\left(\epsilon_{f}\right) d \epsilon_{r} d \epsilon_{f} \\
& +\frac{p_{0} \lambda_{0}}{p_{1}+p_{0} \lambda_{0}} \iint_{-\infty}^{+\infty} \epsilon_{r} \epsilon_{f} \delta\left(\epsilon_{r}\right) U_{\left(-\phi_{0}, 0\right)}\left(\epsilon_{f}\right) d \epsilon_{r} d \epsilon_{f} \\
& +\frac{p_{0} \lambda_{0}}{p_{1}+p_{0} \lambda_{0}} \iint_{-\infty}^{+\infty} \epsilon_{r} \epsilon_{f} \delta\left(\epsilon_{f}\right) U_{\left(0, \phi_{0}\right)}\left(\epsilon_{r}\right) d \epsilon_{r} d \epsilon_{f} \\
= & 0+0+0=0
\end{aligned}
$$

The marginal PDF of $\mathcal{E}_{r}$ and $\mathcal{E}_{f}$ have changed (unlike the case A and all other cases), as well as their expectations and variances. The marginal $P D F$ of $\mathcal{E}_{r}$ is given by

$$
\begin{aligned}
f_{\mathcal{E}_{r}}\left(\epsilon_{r}\right) & =\int_{-\infty}^{+\infty} f_{\mathcal{E}_{r} \mathcal{E}_{f}}\left(\epsilon_{r}, \epsilon_{f}\right) d \epsilon_{f} \\
& =p^{\bullet} \delta\left(\epsilon_{r}\right) \int_{-\infty}^{+\infty} \delta\left(\epsilon_{f}\right) d \epsilon_{f}+p^{*} \delta\left(\epsilon_{r}\right) \int_{-\infty}^{+\infty} U_{\left(-\lambda_{0} \phi_{0}, 0\right)}\left(\epsilon_{f}\right) d \epsilon_{f}+p^{*} U_{\left(0, \lambda_{0} \phi_{0}\right)}\left(\epsilon_{r}\right) \int_{-\infty}^{+\infty} \delta\left(\epsilon_{f}\right) d \epsilon_{f} \\
& =\frac{p_{1}-p_{0} \lambda_{0}}{p_{1}+p_{0} \lambda_{0}} \delta\left(\epsilon_{r}\right)+\frac{p_{0} \lambda_{0}}{p_{1}+p_{0} \lambda_{0}} \delta\left(\epsilon_{r}\right)+\frac{p_{0} \lambda_{0}}{p_{1}+p_{0} \lambda_{0}} U_{\left(0, \lambda_{0} \phi_{0}\right)}\left(\epsilon_{r}\right) \\
& =\frac{p_{1}}{p_{1}+p_{0} \lambda_{0}} \delta\left(\epsilon_{r}\right)+\frac{p_{0} \lambda_{0}}{p_{1}+p_{0} \lambda_{0}} U_{\left(0, \lambda_{0} \phi_{0}\right)}\left(\epsilon_{r}\right)
\end{aligned}
$$

Likewise, the marginal $P D F$ of $\mathcal{E}_{f}$ is given by

$$
f_{\mathcal{E}_{f}}\left(\epsilon_{f}\right)=\frac{p_{1}}{p_{1}+p_{0} \lambda_{0}} \delta\left(\epsilon_{f}\right)+\frac{p_{0} \lambda_{0}}{p_{1}+p_{0} \lambda_{0}} U_{\left(-\lambda_{0} \phi_{0}, 0\right)}\left(\epsilon_{f}\right) .
$$

Their expectations are given by (using result 123)

$$
E\left\{\mathcal{E}_{r}\right\}=\frac{p_{1}}{\left(p_{1}+p_{0} \lambda_{0}\right)} 0+\frac{p_{0} \lambda_{0}}{\left(p_{1}+p_{0} \lambda_{0}\right)} \frac{\lambda_{0} \phi_{0}}{2}=\frac{p_{0} \lambda_{0}}{\left(p_{1}+p_{0} \lambda_{0}\right)} \frac{\lambda_{0} \phi_{0}}{2}=-E\left\{\mathcal{E}_{f}\right\}
$$

and their variances are given by (using result 125)

$$
\begin{aligned}
\operatorname{var}\left\{\mathcal{E}_{r}\right\} & =\frac{p_{1}}{\left(p_{1}+p_{0} \lambda_{0}\right)} 0+\frac{p_{0} \lambda_{0}}{\left(p_{1}+p_{0} \lambda_{0}\right)} \frac{\left(\lambda_{0} \phi_{0}\right)^{2}}{12}+\frac{p_{1}}{\left(p_{1}+p_{0} \lambda_{0}\right)} \frac{p_{0} \lambda_{0}}{\left(p_{1}+p_{0} \lambda_{0}\right)}\left(0-\frac{\lambda_{0} \phi_{0}}{2}\right)^{2} \\
& =\frac{p_{0} \lambda_{0}}{\left(p_{1}+p_{0} \lambda_{0}\right)} \frac{\left(\lambda_{0} \phi_{0}\right)^{2}}{12}+\frac{p_{1} p_{0} \lambda_{0}}{\left(p_{1}+p_{0} \lambda_{0}\right)^{2}} \frac{\left(\lambda_{0} \phi_{0}\right)^{2}}{4} \\
& =\frac{\left(\lambda_{0} \phi_{0}\right)^{2}}{12} \frac{p_{0} \lambda_{0}\left(p_{1}+p_{0} \lambda_{0}\right)+3 p_{1} p_{0} \lambda_{0}}{\left(p_{1}+p_{0} \lambda_{0}\right)^{2}} \\
& =\frac{\left(\lambda_{0} \phi_{0}\right)^{2}}{12} \frac{p_{0} \lambda_{0}\left(4 p_{1}+p_{0} \lambda_{0}\right)}{\left(p_{1}+p_{0} \lambda_{0}\right)^{2}}=\operatorname{var}\left\{\mathcal{E}_{f}\right\}
\end{aligned}
$$

Then, we can compute $C\left\{\mathcal{E}_{r}, \mathcal{E}_{f}\right\}$ for the case 0

$$
C\left\{\mathcal{E}_{r}, \mathcal{E}_{f}\right\}=E\left\{\mathcal{E}_{r}, \mathcal{E}_{f}\right\}-E\left\{\mathcal{E}_{r}\right\} E\left\{\mathcal{E}_{f}\right\}=\left(\frac{p_{0} \lambda_{0}}{\left(p_{1}+p_{0} \lambda_{0}\right)} \frac{\lambda_{0} \phi_{0}}{2}\right)^{2}=\frac{\left(p_{0} \lambda_{0}\right)^{2}}{\left(p_{1}+p_{0} \lambda_{0}\right)^{2}} \frac{\left(\lambda_{0} \phi_{0}\right)^{2}}{4}
$$




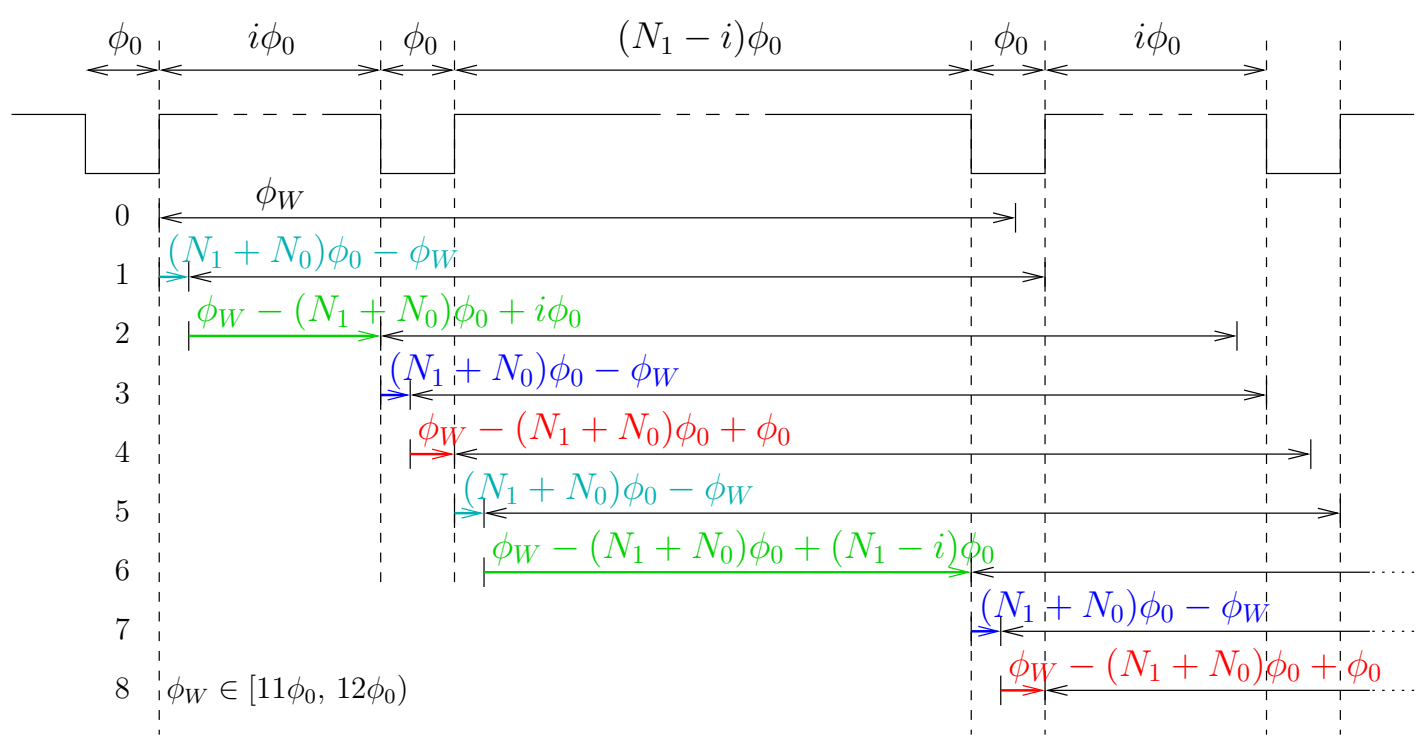

Figure 25: Computation of the probabilities and intervals associated with the case D, and for all codes $\left(\lambda_{d}=0.6\right.$ in the scheme).

and, finally, we obtain the variance of $\Phi_{b}$ for the case 0

$$
\begin{aligned}
\sigma_{\Phi_{b}}^{2} & =\frac{\sigma_{\mathcal{E}_{r}}^{2}+C\left\{\mathcal{E}_{r}, \mathcal{E}_{f}\right\}}{2} \\
& =\frac{\left(\frac{\left(\lambda_{0} \phi_{0}\right)^{2}}{12} \frac{p_{0} \lambda_{0}\left(4 p_{1}+p_{0} \lambda_{0}\right)}{\left(p_{1}+p_{0} \lambda_{0}\right)^{2}}\right)+\left(\frac{\left(p_{0} \lambda_{0}\right)^{2}}{\left(p_{1}+p_{0} \lambda_{0}\right)^{2}} \frac{\left(\lambda_{0} \phi_{0}\right)^{2}}{4}\right)}{2} \\
& =\frac{\left(\lambda_{0} \phi_{0}\right)^{2}}{24}\left[\frac{p_{0} \lambda_{0}\left(4 p_{1}+p_{0} \lambda_{0}\right)+3\left(p_{0} \lambda_{0}\right)^{2}}{\left(p_{1}+p_{0} \lambda_{0}\right)^{2}}\right] \\
& =\frac{\left(\lambda_{0} \phi_{0}\right)^{2}}{24} \frac{4 p_{0} \lambda_{0}}{\left(p_{1}+p_{0} \lambda_{0}\right)} \\
& =p_{0} \frac{\phi_{0}^{2}}{6} \frac{\lambda_{0}^{3}}{\left(p_{1}+p_{0} \lambda_{0}\right)} .
\end{aligned}
$$

\section{A.5 Details for the case D}

The different probabilities are computed as follows: we have to measure the total duration for which the different events occur over a code period, and then divide by the code period. The situation is depicted in Figure 25. The computation of these probabilities is detailed hereafter:

$$
\begin{aligned}
& \phi^{\bullet}=2 \phi_{W}-2\left(N_{1}+N_{0}\right) \phi_{0}+N_{1} \phi_{0} \\
& =2\left[\left(N_{1}+1\right) \phi_{0}+\lambda_{d} \phi_{0}\right]-N_{1} \phi_{0}-2 N_{0} \phi_{0} \\
& =N_{1} \phi_{0}-N_{0} \phi_{0}+N_{0} \lambda_{d} \phi_{0} \\
& =N_{1} \phi_{0}-N_{0} \phi_{0}\left(1-\lambda_{d}\right) \text {. } \\
& p^{\bullet}=\frac{\phi^{\bullet}}{\phi_{\text {duration }}}=\frac{N_{1} \phi_{0}-N_{0} \phi_{0}\left(1-\lambda_{d}\right)}{\left(N_{1}+N_{0}\right) \phi_{0}}=p_{1}-p_{0}\left(1-\lambda_{d}\right) .
\end{aligned}
$$




$$
\begin{aligned}
\phi^{\circ} & =2 \phi_{W}-2\left(N_{1}+N_{0}\right) \phi_{0}+2 \phi_{0} \\
& =2\left[\left(N_{1}+1\right) \phi_{0}+\lambda_{d} \phi_{0}\right]-2 N_{1} \phi_{0}-N_{0} \phi_{0} \\
& =2 N_{1} \phi_{0}+N_{0} \phi_{0}+N_{0} \lambda_{d} \phi_{0}-2 N_{1} \phi_{0}-N_{0} \phi_{0} \\
& =N_{0} \phi_{0} \lambda_{d} . \\
& p^{\circ}=\frac{\phi^{\circ}}{\phi_{\text {duration }}}=\frac{N_{0} \phi_{0} \lambda_{d}}{\left(N_{1}+N_{0}\right) \phi_{0}}=p_{0} \lambda_{d} . \\
\phi^{*} & =2\left(N_{1}+N_{0}\right) \phi_{0}-2 \phi_{W} \\
& =2\left(N_{1}+N_{0}\right) \phi_{0}-2\left[\left(N_{1}+1\right) \phi_{0}+\lambda_{d} \phi_{0}\right] \\
& =N_{0} \phi_{0}-N_{0} \lambda_{d} \phi_{0} \\
& =N_{0} \phi_{0}\left(1-\lambda_{d}\right) . \\
p^{*} & =\frac{\phi^{*}}{\phi_{\text {duration }}}=\frac{N_{0} \phi_{0}\left(1-\lambda_{d}\right)}{\left(N_{1}+N_{0}\right) \phi_{0}}=p_{0}\left(1-\lambda_{d}\right) .
\end{aligned}
$$

We can check that the probabilities sum up to 1

$$
p^{\bullet}+p^{\circ}+2 p^{*}=p_{1}-p_{0}\left(1-\lambda_{d}\right)+p_{0} \lambda_{d}+2 p_{0}\left(1-\lambda_{d}\right)=p_{1}+p_{0}=1 .
$$

The joint expectation is derived as follows

$$
\begin{aligned}
E\left\{\mathcal{E}_{r}, \mathcal{E}_{f}\right\}= & \iint_{-\infty}^{+\infty} \epsilon_{r} \epsilon_{f} f_{\mathcal{E}_{r} \mathcal{E}_{f}}\left(\epsilon_{r}, \epsilon_{f}\right) d \epsilon_{r} d \epsilon_{f} \\
= & p^{\bullet} \iint_{-\infty}^{+\infty} \epsilon_{r} \epsilon_{f} \delta\left(\epsilon_{r}\right) \delta\left(\epsilon_{f}\right) d \epsilon_{r} d \epsilon_{f} \\
& +p^{*} \iint_{-\infty}^{+\infty} \epsilon_{r} \epsilon_{f} \delta\left(\epsilon_{r}\right) U_{\left(-\phi_{0}, \lambda_{d} \phi_{0}\right)}\left(\epsilon_{f}\right) d \epsilon_{r} d \epsilon_{f} \\
& +p^{*} \iint_{-\infty}^{+\infty} \epsilon_{r} \epsilon_{f} \delta\left(\epsilon_{f}\right) U_{\left(\lambda_{d} \phi_{0}, \phi_{0}\right)}\left(\epsilon_{r}\right) d \epsilon_{r} d \epsilon_{f} \\
& +p^{\circ} \iint_{-\infty}^{+\infty} \epsilon_{r} \epsilon_{f} 2 \delta\left(\left(\epsilon_{r}-\epsilon_{f}\right)-\lambda_{d} \phi_{0}\right) U_{\left(-\lambda_{d} \phi_{0}, \lambda_{d} \phi_{0}\right)}\left(\epsilon_{r}+\epsilon_{f}\right) d \epsilon_{r} d \epsilon_{f} \\
= & 0+0+0+p^{\circ}\left(-\frac{\left(\lambda_{d} \phi_{0}\right)^{2}}{6}\right)=-p_{0} \lambda_{d} \frac{\left(\lambda_{d} \phi_{0}\right)^{2}}{6}=-p_{0} \lambda_{d}^{3} \frac{\phi_{0}^{2}}{6} .
\end{aligned}
$$

The covariance is then given by

$$
C\left\{\mathcal{E}_{r}, \mathcal{E}_{f}\right\}=E\left\{\mathcal{E}_{r}, \mathcal{E}_{f}\right\}-E\left\{\mathcal{E}_{r}\right\} E\left\{\mathcal{E}_{f}\right\}=-p_{0} \lambda_{d}^{3} \frac{\phi_{0}^{2}}{6}+p_{0}^{2} \frac{\phi_{0}^{2}}{4} .
$$

So that we can establish the value of the variance as

$$
\begin{aligned}
\sigma_{\Phi_{b}}^{2} & =\frac{\sigma_{\mathcal{E}_{r}}^{2}+C\left\{\mathcal{E}_{r}, \mathcal{E}_{f}\right\}}{2} \\
& =\frac{\left(p_{0} \frac{\phi_{0}^{2}}{3}-p_{0}^{2} \frac{\phi_{0}^{2}}{4}\right)+\left(-p_{0} \lambda_{d}^{3} \frac{\phi_{0}^{2}}{6}+p_{0}^{2} \frac{\phi_{0}^{2}}{4}\right)}{2} \\
& =\frac{p_{0} \frac{\phi_{0}^{2}}{3}-p_{0} \lambda_{d}^{3} \frac{\phi_{0}^{2}}{6}}{2} \\
& =p_{0} \frac{\phi_{0}^{2}}{6} \frac{2-\lambda_{d}^{3}}{2} \\
& =p_{0} \frac{\phi_{0}^{2}}{6}\left(1-\frac{\lambda_{d}^{3}}{2}\right) .
\end{aligned}
$$


The presence of the factor 2 in the fourth term of $f_{\mathcal{E}_{r} \mathcal{E}_{f}}\left(\epsilon_{r}, \epsilon_{f}\right)$ is explained hereafter. This factor is necessary so that the joint $P D F$ integrates to 1 , as explain below

$$
I=\iint_{-\infty}^{+\infty} k \delta\left(\left(\epsilon_{r}-\epsilon_{f}\right)-\lambda_{d} \phi_{0}\right) U_{\left(-\lambda_{d} \phi_{0}, \lambda_{d} \phi_{0}\right)}\left(\epsilon_{r}+\epsilon_{f}\right) d \epsilon_{r} d \epsilon_{f}=1 .
$$

To compute this, we apply this change of variables

$$
\left\{\begin{array}{l}
a=\epsilon_{r}-\epsilon_{f} \\
b=\epsilon_{r}+\epsilon_{f}
\end{array}\right.
$$

or, equivalently,

$$
\left\{\begin{array}{l}
\epsilon_{r}=\frac{a+b}{2} \\
\epsilon_{f}=\frac{b-a}{2}
\end{array}\right.
$$

and the associated Jacobian is equal to

$$
J=\left|\begin{array}{cc}
\frac{\partial \epsilon_{r}}{\partial a} & \frac{\partial \epsilon_{r}}{\partial b} \\
\frac{\partial \epsilon_{f}}{\partial a} & \frac{\partial \epsilon_{f}}{\partial b}
\end{array}\right|=\left|\begin{array}{rr}
\frac{1}{2} & \frac{1}{2} \\
-\frac{1}{2} & \frac{1}{2}
\end{array}\right|=\frac{1}{4}-\left(-\frac{1}{4}\right)=\frac{1}{2} .
$$

So, we have

$$
\begin{aligned}
I & =\iint_{-\infty}^{+\infty} k \delta\left(a-\lambda_{d} \phi_{0}\right) U_{\left(-\lambda_{d} \phi_{0}, \lambda_{d} \phi_{0}\right)}(b)|J| d a d b \\
& =k|J| \int_{-\infty}^{+\infty} \delta\left(a-\lambda_{d} \phi_{0}\right) d a \int_{-\infty}^{+\infty} U_{\left(-\lambda_{d} \phi_{0}, \lambda_{d} \phi_{0}\right)}(b) d b \\
& =k \times \frac{1}{2} \times 1 \times 1=\frac{k}{2} \\
& =1 \Rightarrow k=2 .
\end{aligned}
$$

The computation of the fourth term of $E\left\{\mathcal{E}_{r}, \mathcal{E}_{f}\right\}$ is detailed hereafter

$$
K=\iint_{-\infty}^{+\infty} \epsilon_{r} \epsilon_{f} 2 \delta\left(\left(\epsilon_{r}-\epsilon_{f}\right)-\lambda_{d} \phi_{0}\right) U_{\left(-\lambda_{d} \phi_{0}, \lambda_{d} \phi_{0}\right)}\left(\epsilon_{r}+\epsilon_{f}\right) d \epsilon_{r} d \epsilon_{f} .
$$

By using the same change of variables, we have

$$
\begin{aligned}
K= & \iint_{-\infty}^{+\infty}\left(\frac{a+b}{2}\right)\left(\frac{b-a}{2}\right) 2 \delta\left(a-\lambda_{d} \phi_{0}\right) U_{\left(-\lambda_{d} \phi_{0}, \lambda_{d} \phi_{0}\right)}(b)|J| d a d b \\
& =\iint_{-\infty}^{+\infty}\left(\frac{b^{2}-a^{2}}{4}\right) \delta\left(a-\lambda_{d} \phi_{0}\right) U_{\left(-\lambda_{d} \phi_{0}, \lambda_{d} \phi_{0}\right)}(b) d a d b \\
& =\frac{1}{4} \iint_{-\infty}^{+\infty} b^{2} \delta\left(a-\lambda_{d} \phi_{0}\right) U_{\left(-\lambda_{d} \phi_{0}, \lambda_{d} \phi_{0}\right)}(b) d a d b \\
& -\frac{1}{4} \iint_{-\infty}^{+\infty} a^{2} \delta\left(a-\lambda_{d} \phi_{0}\right) U_{\left(-\lambda_{d} \phi_{0}, \lambda_{d} \phi_{0}\right)}(b) d a d b \\
= & K_{1}-K_{2} .
\end{aligned}
$$

The first term $K_{1}$ is equal to

$$
\begin{aligned}
K_{1} & =\frac{1}{4}\left(\int_{-\infty}^{+\infty} \delta\left(a-\lambda_{d} \phi_{0}\right) d a\right)\left(\int_{-\infty}^{+\infty} b^{2} U_{\left(-\lambda_{d} \phi_{0}, \lambda_{d} \phi_{0}\right)}(b) d b\right) \\
& =\frac{1}{4}(1)\left(\frac{\left(2 \lambda_{d} \phi_{0}\right)^{2}}{12}\right) \\
& =\frac{\left(\lambda_{d} \phi_{0}\right)^{2}}{12} .
\end{aligned}
$$




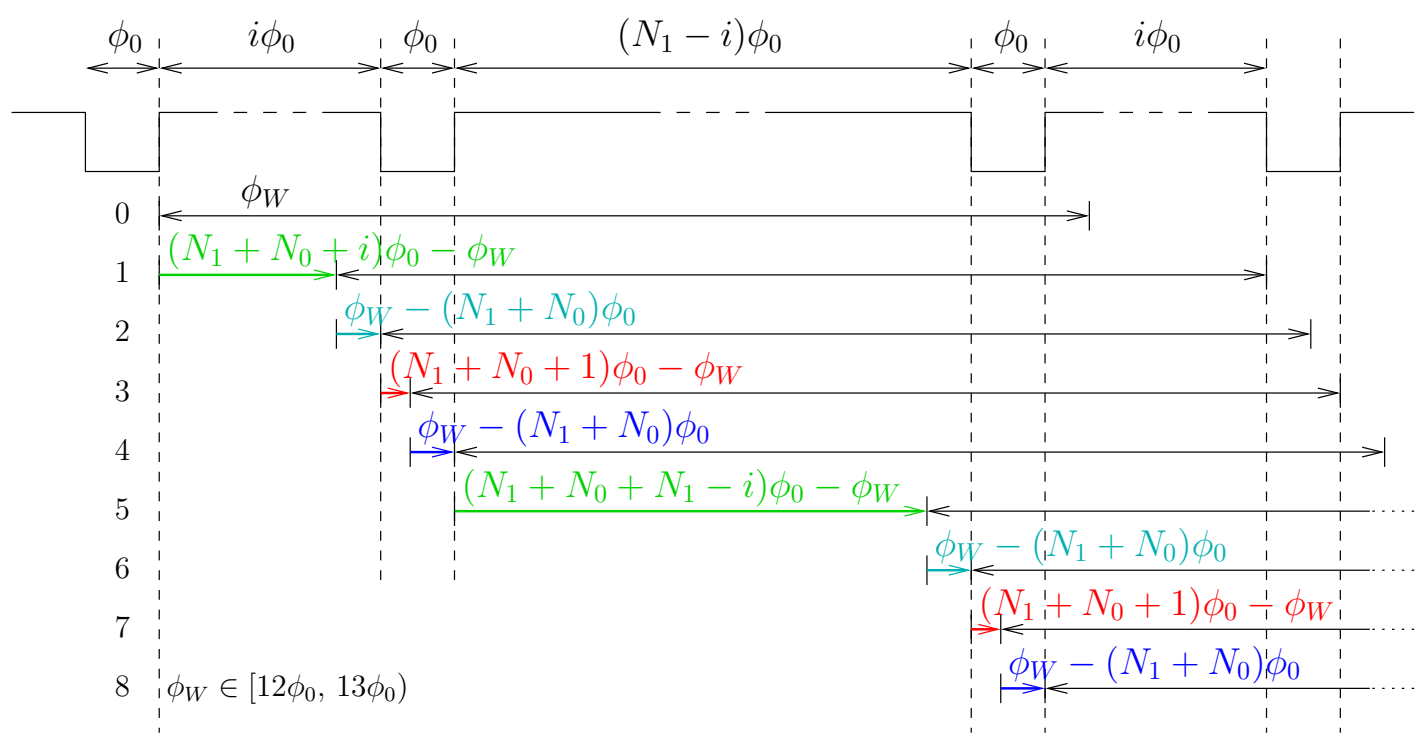

Figure 26: Computation of the probabilities and intervals associated with the case E, and for all codes $\left(\lambda_{e}=0.6\right.$ in the scheme).

The second term $K_{2}$ is equal to

$$
\begin{aligned}
K_{2} & =\frac{1}{4}\left(\int_{-\infty}^{+\infty} a^{2} \delta\left(a-\lambda_{d} \phi_{0}\right) d a\right)\left(\int_{-\infty}^{+\infty} U_{\left(-\lambda_{d} \phi_{0}, \lambda_{d} \phi_{0}\right)}(b) d b\right) \\
& =\frac{1}{4}\left(\lambda_{d} \phi_{0}\right)^{2}(1) \\
& =\frac{\left(\lambda_{d} \phi_{0}\right)^{2}}{4} .
\end{aligned}
$$

And, finally we have

$$
K=K_{1}-K_{2}=\frac{\left(\lambda_{d} \phi_{0}\right)^{2}}{12}-\frac{\left(\lambda_{d} \phi_{0}\right)^{2}}{4}=-\frac{\left(\lambda_{d} \phi_{0}\right)^{2}}{6}=-\frac{\phi_{0}^{2}}{6} \lambda_{d}^{2} .
$$

\section{A.6 Details for the case $\mathrm{E}$}

The different probabilities are computed as follows: we have to measure the total duration for which the different events occur over a code period, and then divide by the code period. The situation is depicted in Figure 26. The computation of these probabilities is detailed hereafter:

$$
\begin{aligned}
\phi^{\bullet} & =\left(2 N_{1}+2 N_{0}+N_{1}\right) \phi_{0}-2 \phi_{W} \\
& =3 N_{1} \phi_{0}+2 N_{0} \phi_{0}-2\left[\left(N_{1}+N_{0}\right) \phi_{0}+\lambda_{e} \phi_{0}\right] \\
& =3 N_{1} \phi_{0}+2 N_{0} \phi_{0}-2 N_{1} \phi_{0}-2 N_{0} \phi_{0}-2 \lambda_{e} \phi_{0} \\
& =N_{1} \phi_{0}-N_{0} \phi_{0} \lambda_{e} . \\
p^{\bullet} & =\frac{\phi^{\bullet}}{\phi_{\text {duration }}}=\frac{N_{1} \phi_{0}-N_{0} \phi_{0} \lambda_{e}}{\left(N_{1}+N_{0}\right) \phi_{0}}=p_{1}-p_{0} \lambda_{e} . \\
\phi^{\circ} & =\left(2 N_{1}+2 N_{0}+2\right) \phi_{0}-2 \phi_{W} \\
& =2 N_{1} \phi_{0}+3 N_{0} \phi_{0}-2\left[\left(N_{1}+N_{0}\right) \phi_{0}+\lambda_{e} \phi_{0}\right] \\
& =N_{0} \phi_{0}-N_{0} \lambda_{e} \phi_{0} \\
& =N_{0} \phi_{0}\left(1-\lambda_{e}\right) .
\end{aligned}
$$




$$
\begin{aligned}
p^{\circ} & =\frac{\phi^{\circ}}{\phi_{\text {duration }}}=\frac{N_{0} \phi_{0}\left(1-\lambda_{e}\right)}{\left(N_{1}+N_{0}\right) \phi_{0}}=p_{0}\left(1-\lambda_{e}\right) . \\
\phi^{*} & =2 \phi_{W}-2\left(N_{1}+N_{0}\right) \phi_{0} \\
& =2\left[\left(N_{1}+N_{0}\right) \phi_{0}+\lambda_{e} \phi_{0}\right]-2\left(N_{1}+N_{0}\right) \phi_{0} \\
& =2 \lambda_{e} \phi_{0} \\
& =N_{0} \phi_{0} \lambda_{e} . \\
& p^{*}=\frac{\phi^{*}}{\phi_{\text {duration }}}=\frac{N_{0} \phi_{0} \lambda_{e}}{\left(N_{1}+N_{0}\right) \phi_{0}}=p_{0} \lambda_{e} .
\end{aligned}
$$

We can check that the probabilities sum up to 1

$$
p^{\bullet}+p^{\circ}+2 p^{*}=p_{1}-p_{0} \lambda_{e}+p_{0}\left(1-\lambda_{e}\right)+2 p_{0} \lambda_{e}=p_{1}+p_{0}=1 .
$$

The joint expectation is derived as follows

$$
\begin{aligned}
E\left\{\mathcal{E}_{r}, \mathcal{E}_{f}\right\}= & \iint_{-\infty}^{+\infty} \epsilon_{r} \epsilon_{f} f_{\mathcal{E}_{r} \mathcal{E}_{f}}\left(\epsilon_{r}, \epsilon_{f}\right) d \epsilon_{r} d \epsilon_{f} \\
= & p^{\bullet} \iint_{-\infty}^{+\infty} \epsilon_{r} \epsilon_{f} \delta\left(\epsilon_{r}\right) \delta\left(\epsilon_{f}\right) d \epsilon_{r} d \epsilon_{f} \\
& +p^{*} \iint_{-\infty}^{+\infty} \epsilon_{r} \epsilon_{f} \delta\left(\epsilon_{r}\right) U_{\left(-\lambda_{e} \phi_{0}, 0\right)}\left(\epsilon_{f}\right) d \epsilon_{r} d \epsilon_{f} \\
& +p^{*} \iint_{-\infty}^{+\infty} \epsilon_{r} \epsilon_{f} \delta\left(\epsilon_{f}\right) U_{\left(0, \lambda_{e} \phi_{0}\right)}\left(\epsilon_{r}\right) d \epsilon_{r} d \epsilon_{f} \\
& +p^{\circ} \iint_{-\infty}^{+\infty} \epsilon_{r} \epsilon_{f} 2 \delta\left(\left(\epsilon_{r}-\epsilon_{f}\right)-\left(1+\lambda_{e}\right) \phi_{0}\right) U_{\left(-\left(1-\lambda_{e}\right) \phi_{0},\left(1-\lambda_{e}\right) \phi_{0}\right)}\left(\epsilon_{r}+\epsilon_{f}\right) d \epsilon_{r} d \epsilon_{f} \\
= & 0+0+0+p^{\circ}\left(-\frac{\phi_{0}^{2}}{6}\left(1+4 \lambda_{e}+\lambda_{e}^{2}\right)\right)=-p_{0} \frac{\phi_{0}^{2}}{6}\left(1-\lambda_{e}\right)\left(1+4 \lambda_{e}+\lambda_{e}^{2}\right) .
\end{aligned}
$$

This leads to the expression of the covariance

$$
C\left\{\mathcal{E}_{r}, \mathcal{E}_{f}\right\}=E\left\{\mathcal{E}_{r}, \mathcal{E}_{f}\right\}-E\left\{\mathcal{E}_{r}\right\} E\left\{\mathcal{E}_{f}\right\}=-p_{0} \frac{\phi_{0}^{2}}{6}\left(1-\lambda_{e}\right)\left(1+4 \lambda_{e}+\lambda_{e}^{2}\right)+p_{0}^{2} \frac{\phi_{0}^{2}}{4} .
$$

The variance is then given by

$$
\begin{aligned}
\sigma_{\Phi_{b}}^{2} & =\frac{\sigma_{\mathcal{E}_{r}}^{2}+C\left\{\mathcal{E}_{r}, \mathcal{E}_{f}\right\}}{2} \\
& =\frac{\left(p_{0} \frac{\phi_{0}^{2}}{3}-p_{0}^{2} \frac{\phi_{0}^{2}}{4}\right)+\left(-p_{0} \frac{\phi_{0}^{2}}{6}\left(1-\lambda_{e}\right)\left(1+4 \lambda_{e}+\lambda_{e}^{2}\right)+p_{0}^{2} \frac{\phi_{0}^{2}}{4}\right)}{2} \\
& =\frac{p_{0} \frac{\phi_{0}^{2}}{3}-p_{0} \frac{\phi_{0}^{2}}{6}\left(1-\lambda_{e}\right)\left(1+4 \lambda_{e}+\lambda_{e}^{2}\right)}{2} \\
& =p_{0} \frac{\phi_{0}^{2}}{6}\left[\frac{2-\left(1-\lambda_{e}\right)\left(1+4 \lambda_{e}+\lambda_{e}^{2}\right)}{2}\right] \\
& =p_{0} \frac{\phi_{0}^{2}}{6}\left(\frac{1-3 \lambda_{e}+3 \lambda_{e}^{2}+\lambda_{e}^{3}}{2}\right) .
\end{aligned}
$$

The explanation about the presence of the factor 2 in the fourth term of $f_{\mathcal{E}_{r} \mathcal{E}_{f}}\left(\epsilon_{r}, \epsilon_{f}\right)$ is the same as for the case D. The computation of the fourth term of $E\left\{\mathcal{E}_{r}, \mathcal{E}_{f}\right\}$ gives

$$
K=\iint_{-\infty}^{+\infty} \epsilon_{r} \epsilon_{f} 2 \delta\left(\left(\epsilon_{r}-\epsilon_{f}\right)-\left(1+\lambda_{e}\right) \phi_{0}\right) U_{\left(-\left(1-\lambda_{e}\right) \phi_{0},\left(1-\lambda_{e}\right) \phi_{0}\right)}\left(\epsilon_{r}+\epsilon_{f}\right) d \epsilon_{r} d \epsilon_{f}
$$


By using the same change of variables (equation (173)), we have

$$
\begin{aligned}
K= & \iint_{-\infty}^{+\infty}\left(\frac{a+b}{2}\right)\left(\frac{b-a}{2}\right) 2 \delta\left(a-\left(1+\lambda_{e}\right) \phi_{0}\right) U_{\left(-\left(1-\lambda_{e}\right) \phi_{0},\left(1-\lambda_{e}\right) \phi_{0}\right)}(b)|J| d a d b \\
= & \iint_{-\infty}^{+\infty}\left(\frac{b^{2}-a^{2}}{4}\right) \delta\left(a-\left(1+\lambda_{e}\right) \phi_{0}\right) U_{\left(-\left(1-\lambda_{e}\right) \phi_{0},\left(1-\lambda_{e}\right) \phi_{0}\right)}(b) d a d b \\
= & \frac{1}{4} \iint_{-\infty}^{+\infty} b^{2} \delta\left(a-\left(1+\lambda_{e}\right) \phi_{0}\right) U_{\left(-\left(1-\lambda_{e}\right) \phi_{0},\left(1-\lambda_{e}\right) \phi_{0}\right)}(b) d a d b \\
& -\frac{1}{4} \iint_{-\infty}^{+\infty} a^{2} \delta\left(a-\left(1+\lambda_{e}\right) \phi_{0}\right) U_{\left(-\left(1-\lambda_{e}\right) \phi_{0},\left(1-\lambda_{e}\right) \phi_{0}\right)}(b) d a d b \\
= & K_{1}-K_{2} .
\end{aligned}
$$

The first term $K_{1}$ is equal to

$$
\begin{aligned}
K_{1} & =\frac{1}{4}\left(\int_{-\infty}^{+\infty} \delta\left(a-\left(1+\lambda_{e}\right) \phi_{0}\right) d a\right)\left(\int_{-\infty}^{+\infty} b^{2} U_{\left(-\left(1-\lambda_{e}\right) \phi_{0},\left(1-\lambda_{e}\right) \phi_{0}\right)}(b) d b\right) \\
& =\frac{1}{4}(1)\left(\frac{\left(2\left(1-\lambda_{e}\right) \phi_{0}\right)^{2}}{12}\right) \\
& =\frac{\left(\left(1-\lambda_{e}\right) \phi_{0}\right)^{2}}{12} .
\end{aligned}
$$

The second term $K_{2}$ is equal to

$$
\begin{aligned}
K_{2} & =\frac{1}{4}\left(\int_{-\infty}^{+\infty} a^{2} \delta\left(a-\left(1+\lambda_{e}\right) \phi_{0}\right) d a\right)\left(\int_{-\infty}^{+\infty} U_{\left(-\left(1-\lambda_{e}\right) \phi_{0},\left(1-\lambda_{e}\right) \phi_{0}\right)}(b) d b\right) \\
& =\frac{1}{4}\left(\left(1+\lambda_{e}\right) \phi_{0}\right)^{2}(1) \\
& =\frac{\left(\left(1+\lambda_{e}\right) \phi_{0}\right)^{2}}{4} .
\end{aligned}
$$

And, finally, we have

$$
\begin{aligned}
K=K_{1}-K_{2} & =\frac{\left(\left(1-\lambda_{e}\right) \phi_{0}\right)^{2}}{12}-\frac{\left(\left(1+\lambda_{e}\right) \phi_{0}\right)^{2}}{4} \\
& =\frac{\phi_{0}^{2}}{12}\left[\left(1-\lambda_{e}\right)^{2}-3\left(1+\lambda_{e}\right)^{2}\right] \\
& =\frac{\phi_{0}^{2}}{12}\left(-2-8 \lambda_{e}-2 \lambda_{e}^{2}\right) \\
& =-\frac{\phi_{0}^{2}}{6}\left(1+4 \lambda_{e}+\lambda_{e}^{2}\right) .
\end{aligned}
$$

\section{A.7 Details for the case B}

The different probabilities are computed as follows: we have to measure the total duration for which the different events occur over a code period, and then divide by the code period. The situation is depicted in Figure 27. The computation of these probabilities is detailed hereafter:

$$
\begin{aligned}
\phi^{\bullet} & =\phi_{W}-\phi_{0}+\left(N_{1}-i\right) \phi_{0}-\phi_{W}+\phi_{W}-\phi_{0} \\
& =\phi_{W}+\left(N_{1}-i-2\right) \phi_{0} \\
& =\left(i \phi_{0}+\lambda_{b} \phi_{0}\right)+\left(N_{1}-i-N_{0}\right) \phi_{0} \\
& =N_{1} \phi_{0}-\frac{N_{0}}{2} \phi_{0}\left(2-\lambda_{b}\right) .
\end{aligned}
$$




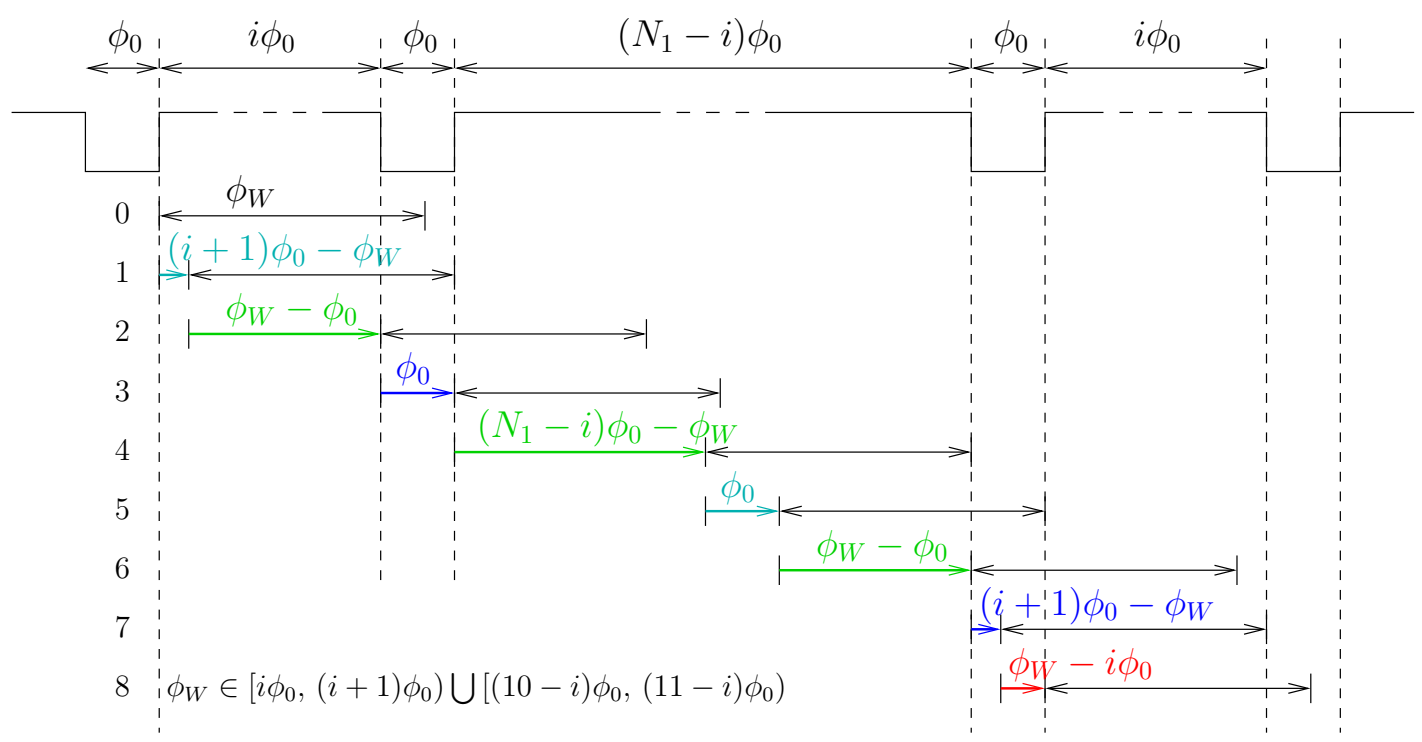

Figure 27: Computation of the probabilities and intervals associated with the case $\mathrm{B}$, and for all codes, except the code $5\left(\lambda_{b}=0.6\right.$ in the scheme).

$$
\begin{aligned}
& p^{\bullet}=\frac{\phi^{\bullet}}{\phi_{\text {duration }}}=\frac{N_{1} \phi_{0}-\frac{N_{0}}{2} \phi_{0}\left(2-\lambda_{b}\right)}{\left(N_{1}+N_{0}\right) \phi_{0}}=p_{1}-\frac{p_{0}}{2}\left(2-\lambda_{b}\right) . \\
& \phi^{\circ}=\phi_{W}-i \phi_{0} \\
& =\left(i \phi_{0}+\lambda_{b} \phi_{0}\right)-i \phi_{0} \\
& =\frac{N_{0}}{2} \phi_{0} \lambda_{b} \text {. } \\
& p^{\circ}=\frac{\phi^{\circ}}{\phi_{\text {duration }}}=\frac{\frac{N_{0}}{2} \phi_{0} \lambda_{b}}{\left(N_{1}+N_{0}\right) \phi_{0}}=\frac{p_{0}}{2} \lambda_{b} . \\
& \phi^{\star}=\phi_{0} \\
& =\frac{N_{0}}{2} \phi_{0} \text {. } \\
& p^{\star}=\frac{\phi^{\star}}{\phi_{\text {duration }}}=\frac{\frac{N_{0}}{2} \phi_{0}}{\left(N_{1}+N_{0}\right) \phi_{0}}=\frac{p_{0}}{2} \text {. } \\
& \phi^{*}=(i+1) \phi_{0}-\phi_{W} \\
& =(i+1) \phi_{0}-\left(i \phi_{0}+\lambda_{b} \phi_{0}\right) \\
& =\phi_{0}-\lambda_{b} \phi_{0} \\
& =\frac{N_{0}}{2} \phi_{0}\left(1-\lambda_{b}\right) \text {. } \\
& p^{*}=\frac{\phi^{*}}{\phi_{\text {duration }}}=\frac{\frac{N_{0}}{2} \phi_{0}\left(1-\lambda_{b}\right)}{\left(N_{1}+N_{0}\right) \phi_{0}}=\frac{p_{0}}{2}\left(1-\lambda_{b}\right) .
\end{aligned}
$$

We can check that the probabilities sum up to 1

$$
p^{\bullet}+p^{\circ}+2 p^{\star}+2 p^{*}=p_{1}-\frac{p_{0}}{2}\left(2-\lambda_{b}\right)+\frac{p_{0}}{2} \lambda_{b}+2 \frac{p_{0}}{2}+2 \frac{p_{0}}{2}\left(1-\lambda_{b}\right)=p_{1}+p_{0}=1 .
$$


The joint expectation is expressed as

$$
\begin{aligned}
E\left\{\mathcal{E}_{r}, \mathcal{E}_{f}\right\}= & \iint_{-\infty}^{+\infty} \epsilon_{r} \epsilon_{f} f_{\mathcal{E}_{r} \mathcal{E}_{f}}\left(\epsilon_{r}, \epsilon_{f}\right) d \epsilon_{r} d \epsilon_{f} \\
= & p^{\bullet} \iint_{-\infty}^{+\infty} \epsilon_{r} \epsilon_{f} \delta\left(\epsilon_{r}\right) \delta\left(\epsilon_{f}\right) d \epsilon_{r} d \epsilon_{f} \\
& +p^{\star} \iint_{-\infty}^{+\infty} \epsilon_{r} \epsilon_{f} \delta\left(\epsilon_{r}\right) U_{\left(-\phi_{0}, 0\right)}\left(\epsilon_{f}\right) d \epsilon_{r} d \epsilon_{f} \\
& +p^{\star} \iint_{-\infty}^{+\infty} \epsilon_{r} \epsilon_{f} \delta\left(\epsilon_{f}\right) U_{\left(0, \phi_{0}\right)}\left(\epsilon_{r}\right) d \epsilon_{r} d \epsilon_{f} \\
& +p^{*} \iint_{-\infty}^{+\infty} \epsilon_{r} \epsilon_{f} \delta\left(\epsilon_{r}\right) U_{\left(-\phi_{0}, \lambda_{b} \phi_{0}\right)}\left(\epsilon_{f}\right) d \epsilon_{r} d \epsilon_{f} \\
& +p^{*} \iint_{-\infty}^{+\infty} \epsilon_{r} \epsilon_{f} \delta\left(\epsilon_{f}\right) U_{\left(\lambda_{b} \phi_{0}, \phi_{0}\right)}\left(\epsilon_{r}\right) d \epsilon_{r} d \epsilon_{f} \\
& +p^{\circ} \iint_{-\infty}^{+\infty} \epsilon_{r} \epsilon_{f} 2 \delta\left(\left(\epsilon_{r}-\epsilon_{f}\right)-\lambda_{b} \phi_{0}\right) U_{\left(-\lambda_{b} \phi_{0}, \lambda_{b} \phi_{0}\right)}\left(\epsilon_{r}+\epsilon_{f}\right) d \epsilon_{r} d \epsilon_{f} \\
= & 0+0+0+0+0+p^{\circ}\left(-\frac{\left(\lambda_{b} \phi_{0}\right)^{2}}{6}\right)=-\frac{p_{0}}{2} \lambda_{b} \frac{\left(\lambda_{b} \phi_{0}\right)^{2}}{6}=-p_{0} \lambda_{b}^{3} \frac{\phi_{0}^{2}}{12}
\end{aligned}
$$

Then we derive the covariance of $\mathcal{E}_{r}$ and $\mathcal{E}_{f}$

$$
C\left\{\mathcal{E}_{r}, \mathcal{E}_{f}\right\}=E\left\{\mathcal{E}_{r}, \mathcal{E}_{f}\right\}-E\left\{\mathcal{E}_{r}\right\} E\left\{\mathcal{E}_{f}\right\}=-p_{0} \lambda_{b}^{3} \frac{\phi_{0}^{2}}{12}+p_{0}^{2} \frac{\phi_{0}^{2}}{4} .
$$

This leads to the following expression of the variance:

$$
\begin{aligned}
\sigma_{\Phi_{b}}^{2} & =\frac{\sigma_{\mathcal{E}_{r}}^{2}+C\left\{\mathcal{E}_{r}, \mathcal{E}_{f}\right\}}{2} \\
& =\frac{\left(p_{0} \frac{\phi_{0}^{2}}{3}-p_{0}^{2} \frac{\phi_{0}^{2}}{4}\right)+\left(-p_{0} \lambda_{b}^{3} \frac{\phi_{0}^{2}}{12}+p_{0}^{2} \frac{\phi_{0}^{2}}{4}\right)}{2} \\
& =\frac{p_{0} \frac{\phi_{0}^{2}}{3}-p_{0} \lambda_{b}^{3} \frac{\phi_{0}^{2}}{12}}{2} \\
& =p_{0} \frac{\phi_{0}^{2}}{6}\left(1-\frac{\lambda_{b}^{3}}{4}\right)
\end{aligned}
$$

\section{A.8 Details for the case C}

The different probabilities are computed as follows: we have to measure the total duration for which the different events occur over a code period, and then divide by the code period. The situation is depicted in Figure 28. The computation of these probabilities is detailed hereafter:

$$
\begin{aligned}
\phi^{\bullet} & =i \phi_{0}+\left(N_{1}-i\right) \phi_{0}-\phi_{W}+i \phi_{0} \\
& =N_{1} \phi_{0}+i \phi_{0}-\phi_{W} \\
& =N_{1} \phi_{0}+i \phi_{0}-\left((i+1) \phi_{0}+\lambda_{c} \phi_{0}\right) \\
& =N_{1} \phi_{0}-\frac{N_{0}}{2} \phi_{0}\left(1+\lambda_{c}\right) . \\
p^{\bullet}=\frac{\phi^{\bullet}}{\phi_{\text {duration }}} & =\frac{N_{1} \phi_{0}-\frac{N_{0}}{2} \phi_{0}\left(1+\lambda_{c}\right)}{\left(N_{1}+N_{0}\right) \phi_{0}}=p_{1}-\frac{p_{0}}{2}\left(1+\lambda_{c}\right) .
\end{aligned}
$$




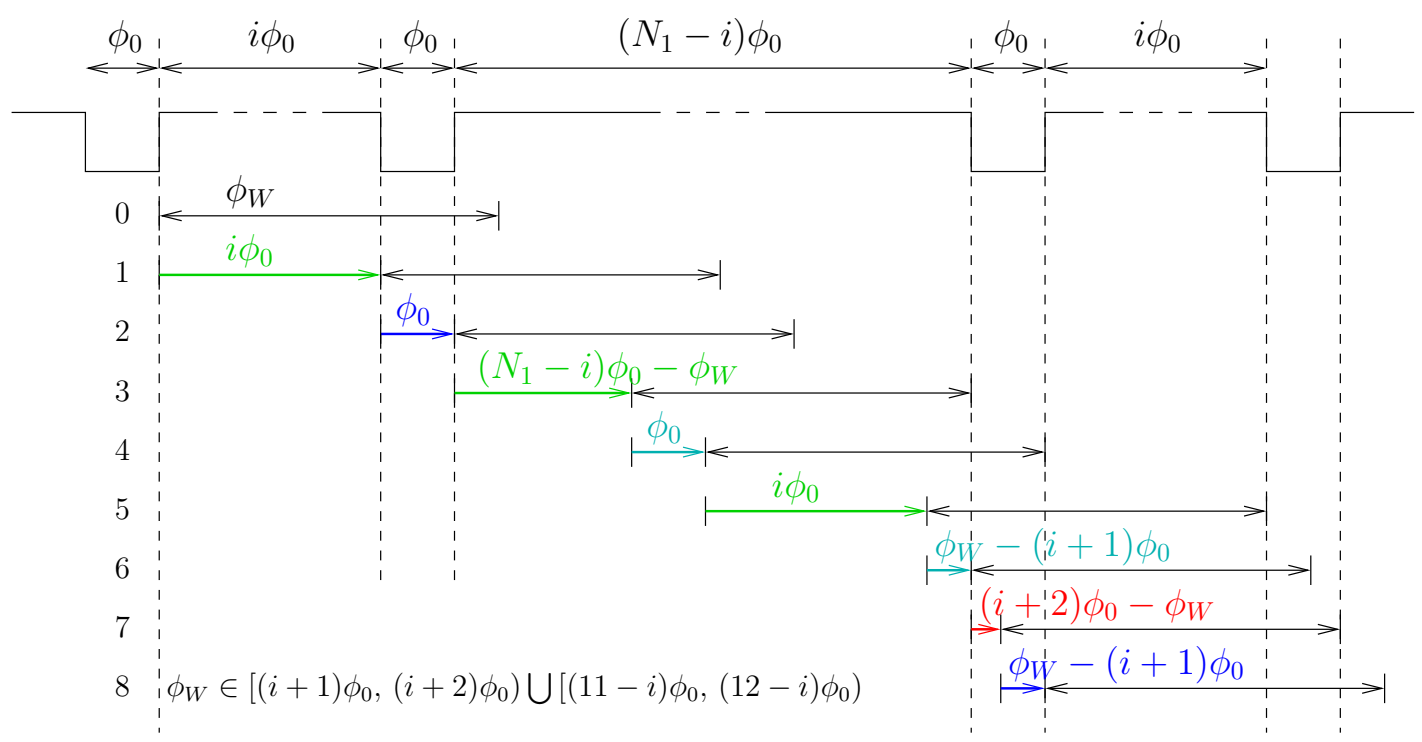

Figure 28: Computation of the probabilities and intervals associated with the case $\mathrm{C}$, and for all codes, except the code 5 ( $\lambda_{c}=0.6$ in the scheme).

$$
\begin{aligned}
& \phi^{\circ}=(i+2) \phi_{0}-\phi_{W} \\
&=i \phi_{0}+2 \phi_{0}-\left((i+1) \phi_{0}+\lambda_{c} \phi_{0}\right) \\
&=\phi_{0}-\lambda_{c} \phi_{0} \\
&=\frac{N_{0}}{2} \phi_{0}\left(1-\lambda_{c}\right) . \\
& p^{\circ}=\frac{\phi^{\circ}}{\phi_{\text {duration }}}=\frac{\frac{N_{0}}{2} \phi_{0}\left(1-\lambda_{c}\right)}{\left(N_{1}+N_{0}\right) \phi_{0}}=\frac{p_{0}}{2}\left(1-\lambda_{c}\right) . \\
& \quad \phi^{\star}=\frac{\phi_{0}}{2} \phi_{0} . \\
& p^{\star}=\frac{\phi^{\star}}{\phi_{\text {duration }}}=\frac{\frac{N_{0}}{2} \phi_{0}}{\left(N_{1}+N_{0}\right) \phi_{0}}=\frac{p_{0}}{2} . \\
& \phi^{*}=\phi_{W}-(i+1) \phi_{0} \\
&=\left((i+1) \phi_{0}+\lambda_{c} \phi_{0}\right)-(i+1) \phi_{0} \\
&=\lambda_{c} \phi_{0} \\
&=\frac{N_{0}}{2} \phi_{0} \lambda_{c} . \\
& p^{*}= \frac{\phi^{*}}{\phi_{\text {duration }}}=\frac{\frac{N_{0}}{2} \phi_{0} \lambda_{c}}{\left(N_{1}+N_{0}\right) \phi_{0}}=\frac{p_{0}}{2} \lambda_{c} .
\end{aligned}
$$

We can check that the probabilities sum up to 1

$$
p^{\bullet}+p^{\circ}+2 p^{\star}+2 p^{*}=p_{1}-\frac{p_{0}}{2}\left(1+\lambda_{c}\right)+\frac{p_{0}}{2}\left(1-\lambda_{c}\right)+2 \frac{p_{0}}{2}+2 \frac{p_{0}}{2} \lambda_{c}=p_{1}+p_{0}=1 .
$$


The joint expectation is expressed as

$$
\begin{aligned}
E\left\{\mathcal{E}_{r}, \mathcal{E}_{f}\right\}= & \iint_{-\infty}^{+\infty} \epsilon_{r} \epsilon_{f} f_{\mathcal{E}_{r} \mathcal{E}_{f}}\left(\epsilon_{r}, \epsilon_{f}\right) d \epsilon_{r} d \epsilon_{f} \\
= & p^{\bullet} \iint_{-\infty}^{+\infty} \epsilon_{r} \epsilon_{f} \delta\left(\epsilon_{r}\right) \delta\left(\epsilon_{f}\right) d \epsilon_{r} d \epsilon_{f} \\
& +p^{\star} \iint_{-\infty}^{+\infty} \epsilon_{r} \epsilon_{f} \delta\left(\epsilon_{r}\right) U_{\left(-\phi_{0}, 0\right)}\left(\epsilon_{f}\right) d \epsilon_{r} d \epsilon_{f} \\
& +p^{\star} \iint_{-\infty}^{+\infty} \epsilon_{r} \epsilon_{f} \delta\left(\epsilon_{f}\right) U_{\left(0, \phi_{0}\right)}\left(\epsilon_{r}\right) d \epsilon_{r} d \epsilon_{f} \\
& +p^{*} \iint_{-\infty}^{+\infty} \epsilon_{r} \epsilon_{f} \delta\left(\epsilon_{r}\right) U_{\left(-\lambda_{c} \phi_{0}, 0\right)}\left(\epsilon_{f}\right) d \epsilon_{r} d \epsilon_{f} \\
& +p^{*} \iint_{-\infty}^{+\infty} \epsilon_{r} \epsilon_{f} \delta\left(\epsilon_{f}\right) U_{\left(0, \lambda_{c} \phi_{0}\right)}\left(\epsilon_{r}\right) d \epsilon_{r} d \epsilon_{f} \\
& +p^{\circ} \iint_{-\infty}^{+\infty} \epsilon_{r} \epsilon_{f} 2 \delta\left(\left(\epsilon_{r}-\epsilon_{f}\right)-\left(1+\lambda_{c}\right) \phi_{0}\right) U_{\left(-\left(1-\lambda_{c}\right) \phi_{0},\left(1-\lambda_{c}\right) \phi_{0}\right)}\left(\epsilon_{r}+\epsilon_{f}\right) d \epsilon_{r} d \epsilon_{f} \\
= & 0+0+0+0+0+p^{\circ}\left(-\frac{\phi_{0}^{2}}{6}\left(1+4 \lambda_{c}+\lambda_{c}^{2}\right)\right) \\
= & -\frac{p_{0}}{2}\left(1-\lambda_{c}\right) \frac{\phi_{0}^{2}}{6}\left(1+4 \lambda_{c}+\lambda_{c}^{2}\right) \\
= & -p_{0} \frac{\phi_{0}^{2}}{6} \frac{\left(1-\lambda_{c}\right)\left(1+4 \lambda_{c}+\lambda_{c}^{2}\right)}{2} .(217)
\end{aligned}
$$

Then we derive the covariance of $\mathcal{E}_{r}$ and $\mathcal{E}_{f}$

$$
C\left\{\mathcal{E}_{r}, \mathcal{E}_{f}\right\}=E\left\{\mathcal{E}_{r}, \mathcal{E}_{f}\right\}-E\left\{\mathcal{E}_{r}\right\} E\left\{\mathcal{E}_{f}\right\}=-p_{0} \frac{\phi_{0}^{2}}{6} \frac{\left(1-\lambda_{c}\right)\left(1+4 \lambda_{c}+\lambda_{c}^{2}\right)}{2}+p_{0}^{2} \frac{\phi_{0}^{2}}{4},
$$

and, finally, the variance:

$$
\begin{aligned}
\sigma_{\Phi_{b}}^{2} & =\frac{\sigma_{\mathcal{E}_{r}}^{2}+C\left\{\mathcal{E}_{r}, \mathcal{E}_{f}\right\}}{2} \\
& =\frac{\left(p_{0} \frac{\phi_{0}^{2}}{3}-p_{0}^{2} \frac{\phi_{0}^{2}}{4}\right)+\left(-p_{0} \frac{\phi_{0}^{2}}{6} \frac{\left(1-\lambda_{c}\right)\left(1+4 \lambda_{c}+\lambda_{c}^{2}\right)}{2}+p_{0}^{2} \frac{\phi_{0}^{2}}{4}\right)}{2} \\
& \left.=\frac{p_{0} \frac{\phi_{0}^{2}}{3}-p_{0} \frac{\phi_{0}^{2}}{6} \frac{\left(1-\lambda_{c}\right)\left(1+4 \lambda_{c}+\lambda_{c}^{2}\right)}{2}}{2}\right) \\
& =p_{0} \frac{\phi_{0}^{2}}{6}\left(\frac{2-\frac{\left(1-\lambda_{c}\right)\left(1+4 \lambda_{c}+\lambda_{c}^{2}\right)}{2}}{2}\right) \\
& =p_{0} \frac{\phi_{0}^{2}}{6}\left(\frac{4-1-4 \lambda_{c}-\lambda_{c}^{2}+\lambda_{c}+4 \lambda_{c}^{2}+\lambda_{c}^{3}}{4}\right) \\
& =p_{0} \frac{\phi_{0}^{2}}{6}\left(\frac{3-3 \lambda_{c}+3 \lambda_{c}^{2}+\lambda_{c}^{3}}{4}\right) .
\end{aligned}
$$




\section{References}

[1] A. Papoulis. Probability, random variables, and stochastic processes. McGraw-Hill, 1991.

[2] V. Pierlot and M. Van Droogenbroeck. A simple and low cost angle measurement system for mobile robot positioning. In Workshop on Circuits, Systems and Signal Processing (ProRISC), pages 251-254, Veldhoven, The Netherlands, November 2009.

[3] V. Pierlot and M. Van Droogenbroeck. Analysis of a robot positioning system based on a rotating receiver, beacons, and coded signals. In European Signal Processing Conference (EUSIPCO), pages 1766-1770, Barcelona, Spain, August 2011.

[4] V. Pierlot and M. Van Droogenbroeck. BeAMS: a beacon based angle measurement sensor for mobile robot positioning. IEEE Transactions on Robotics, 30(3):533-549, June 2014.

[5] V. Pierlot, M. Van Droogenbroeck, and M. Urbin-Choffray. A new three object triangulation algorithm based on the power center of three circles. In Research and Education in Robotics (EUROBOT), volume 161 of Communications in Computer and Information Science, pages 248-262. Springer, 2011. 